Helonal Bureau of Standards

IIbrary, N.W. Blag

JAN 71963
Reference book not to be taken from the library.

\title{
PART A
}

\section{IONOSPHERIC DATA}

ISSUED

DEGEMBER 1962

SEE PAGE 55 FOR INDEX OF OBSERVED

IONOSPHERIC DATA BEGINNING JANUARY 1957

U. S. DEPARTMENT OF COMMERCE

NATIONAL BUREAU OF STANDARDS

CENTRAL RADIO PROPAGATION LABORATORY BOULDER, COLORADO 



\title{
IONOSPHERIC DATA
}

\author{
CONTENTS
}

Page

Ionospheric Data (revised text) . . . . . . . . . . ii

Table of Smoothed Observed Zurich Sunspot Numbers . . . . . iii

World-Wide Sources of Ionospheric Data . . . . . . . . . iv

Tables of Ionospheric Data . . . . . . . . . . . 1

Graphs of Ionospheric Data . . . . . . . . . . 26

Index of Tables and Graphs of Ionospheric

Data in CRPL-F220 (Part A) . . . . . . . . . 51

Index by Issue Number of Observed Ionospheric Data Beginning with January 1957 Published in the CRPL-F(Part A) . . . 55

Index by Issue Number of Ionospheric Data Observed Prior to 1957 Published in 1962 (CRPL-F209(Part A) through -F220 (Part A) . . . . . . . . . . . 58 


\section{IONOSPHERIC DATA}

The CRPL-F series bulletins are issued as part of the responsibility of the Central Radio Propagation Laboratory for the exchange and dissemination of ionospheric and related geophysical data. While originally a by-product of the collection of data by the CRPL for use in radio propagation studies, the CRPL-F series bulletins, Part A, "Ionospheric Data," and Part B, "Solar-Geophysical Data," have provided useful service by collecting and making available a wide variety of data in convenient form for use in research, not only on radio propagation and the ionosphere, but also on a wide variety of geophysical problems. Beginning with CRPL-F21I, Part A, "Ionospheric Data," a number of changes have been made in the tables of ionospheric data which, by providing more information, should increase their usefulness.

The current form of the tables of ionospheric data provides the monthly medians and, in addition, the number of values entering into median determination (count) for all ionospheric characteristics 1isted. Also, the upper and lower quartile values, indicated by UQ and IQ in the tables, are listed for foF2, $h^{\circ} F 2, h^{\prime} F$, and $M(3000) F 2$. Quartile values are not Iisted for the other characteristics because of space limitations. The tables are prepared by IBM machine nethods, which, by improving the speed and efficiency of preparation, permit earlier publication of the data.

Graphs of critical frequencies and M(3000)F2 wi11 continue to appear. Graphs of percentage of time of occurrence for fEs and virtual heights of the regular ionospheric layers are no longer included. This change was necessary to provide space for the enlarged tables. Data on percentage of time of occurrence of fEs above 3,5, and 7 Mc axe still available from the CRPI and the IGY WorId Data Center A for Airglow and Ionosphere.

For many years, the tables of ionospheric data appearing in the $\mathrm{F}$ series, Part A, listed values of medians recomputed at CRPI. While this practice enforced a certain uniformity, it was subject so some valid criticism for tampering with original data. The tables and graphs now show the ionospheric data just as they are provided by the originating laboratory. Responsibility for the accuracy and reliability of the data now rests entirely with the originator.

Gaps in the tables when data normally might be expected indicate the data were not provided by the originator. Following the recommendation of the World-Wide Soundings Committee, only values of median foEs are listed. In the few cases where IEs is still reported instead of foEs, the data will not be printed. Data will appear in the $F$ series, Part $A$, only when the complete daily-hourly tabulations have been received by the CRPI or the IGY World Data Center A for Airglow and Ionosphere. 
Information on symbols, terminology, and conventions may be found in the "URSI Handbook of Ionogram Interpretation and Reduction, of the World-Wide Soundings Committee," edited by W. R. Piggott and K. Rawer (E1sevier, 1961), which supersedes previous documents. A list of symbols is available from CRPL on request.

The following table contains the latest available information on smoothed observed Zurich sunspot numbers, beginning with the minimum of April 1954. Final numbers are 1 isted through June 1961, the succeeding values being based on provisional data.

Smoothed Observed Zurich Sunspot Number

\begin{tabular}{|c|c|c|c|c|c|c|c|c|c|c|c|c|}
\hline Month & Jan & $\mathrm{Feb}$ & Mar. & Apr. & May & Jun & Ju1 & Aug & Sep & Oct. & Nov. & Dec. \\
\hline 1954 & & & & 3 & 4 & 4 & 5 & 7 & 8 & 8 & 9 & 12 \\
\hline 1955 & 14 & 16 & 19 & 23 & 29 & 35 & 40 & 46 & 55 & 64 & 73 & 81 \\
\hline 1956 & 89 & 98 & 109 & 119 & 127 & 137 & 146 & 150 & 151 & 156 & 160 & 164 \\
\hline 1957 & 170 & 172 & 174 & 181 & 186 & 188 & 191 & 194 & 197 & 200 & 201 & 200 \\
\hline 1958 & 199 & 201 & 201 & 197 & 191 & 187 & 185 & 185 & 184 & 182 & 181 & 180 \\
\hline 1959 & 179 & 177 & 174 & 169 & 165 & 161 & 156 & 151 & 146 & 141 & 137 & 132 \\
\hline 1960 & 129 & 125 & 122 & 120 & 117 & 114 & 109 & 102 & 98 & 93 & 88 & 84 \\
\hline 1961 & 80 & 75 & 69 & 64 & 60 & 56 & 53 & 52 & 52 & 51 & 50 & 48 \\
\hline 1962 & 44 & 41 & 39 & 38 & 38 & & & & & & & \\
\hline
\end{tabular}

\section{Units of Ionospheric Data Tables}

foF2, foEs - - Tenths of a megacycle

foF1, FoE - - Hundredths of a megacycle

h'F2, h'F, h'E - Kilometers

(M3000)F2 - - Hundredths

NOTE: Occasionally, when the median falls between two of the observed values, the median is carried an extra decimal place beyond these units. Those cases are easily identifiable by the extra digit appearing to the right of the number, in a column usually left blank.

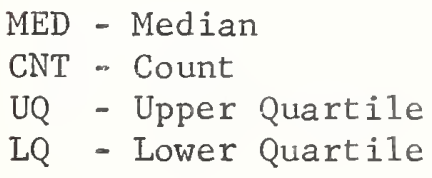




\section{WORLD - WIDE SOURCES OF IONOSPHERIC DATA}

The ionospheric data given here in tables 1 to 100 and figures 1 to 100 were assembled by the Central Radio Propagation Laboratory for analysis and correlation, incidental to CRPL prediction of radio propagation conditions. The data are median values unless otherwise indicated. The following are the sources of the data in this issue:

Meteorological Service, Province of Macau, Asia:

Macau

Comnonwealth of Australia, Ionospheric Prediction Service of the

Commonwealth Observatory:

Brisbane, Australia

Canberra, Australia

Hobart, Tasmania

Mawson

University of Graz:

Graz, Austria

Defence Research Board, Canada:

St. John's, Newfoundland

Radio Wave Research Laboratories, National Taiwan University, Taipeh, Formosa, China:

Formosa, China

Heinrich Hertz Institute, German Academy of Sciences, Berlin:

Juliusruh/Rugen, Germany

Icelandic Post and Telegraph Administration:

Reykjavik, Iceland

Indian Council of Scientific and Industrial Research, Radio Research

Committee, New Delhi, India:

Ahmedabad (Physical Research Laboratory)

Bombay (Al1 India Radio)

Calcutta (Institute of Radio Physics and Electronics)

Delhi (All India Radio)

Kodaikanal (India Meteorological Department)

Madras (A11 India Radio)

Tiruchy (A11 India Radio)

Trivandrun (All India Radio) 
Christchurch Geophysical Observatory, New Zealand Department of Scientific and Industrial Research:

Cape Hallett (Adare), Antarctica

Scott Base, Antarctica

Research Institute of National Defence, Stockholm, Sweden: Lycksele, Sweden

Post, Telephone and Telegraph Administration, Berne, Switzerland: Sottens, Switzerland

United States Army Signal Corps:

Ft. Monnouth, New Jersey

Thule, Greenland

White Sands, New Mexico

National Bureau of Standards (Central Radio Propagation Laboratory):

Fairbanks (College), Alaska (Geophysical Institute of the University of Alaska)

Maui, Hawaii

Washington, D. C. 


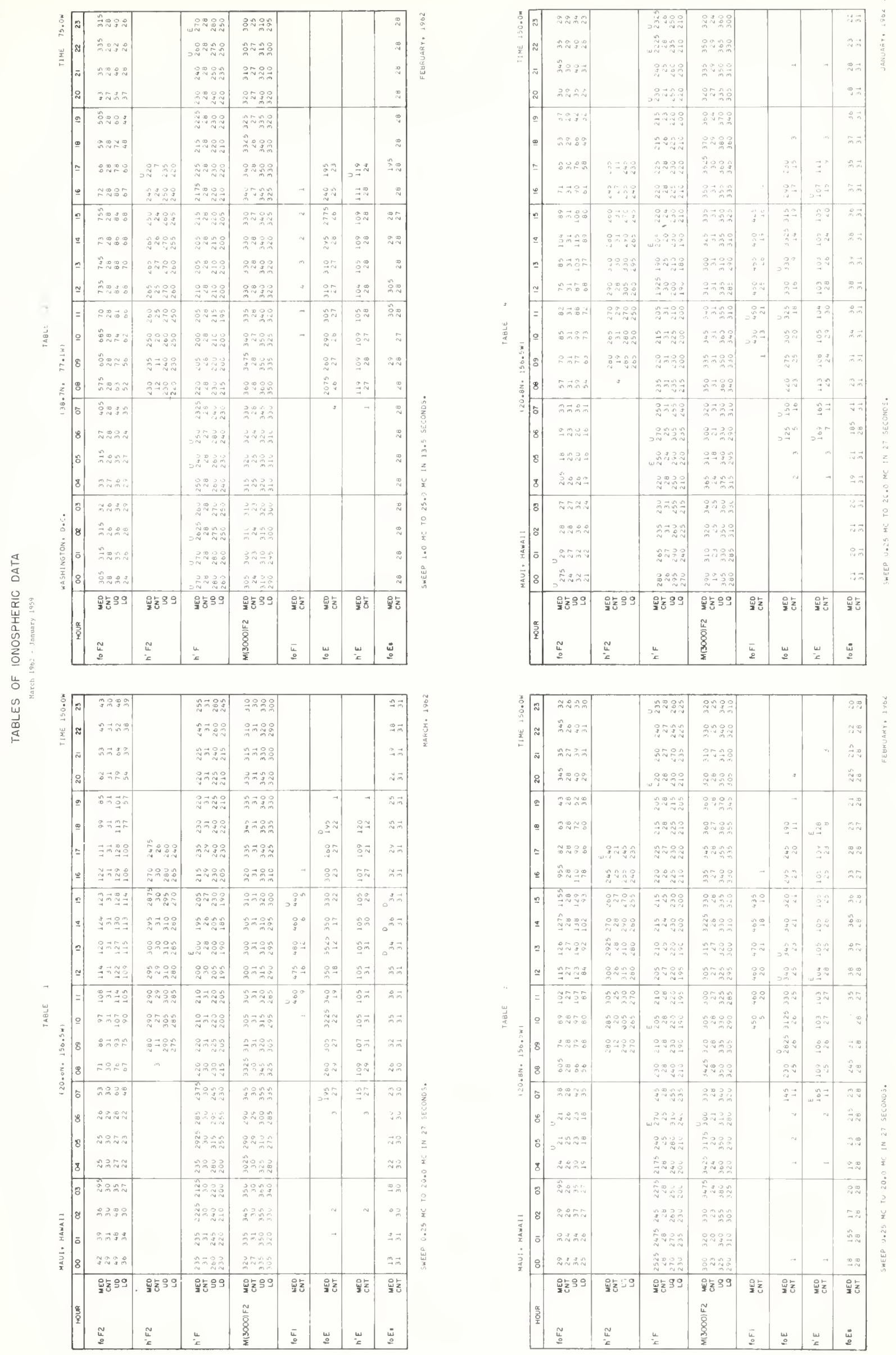

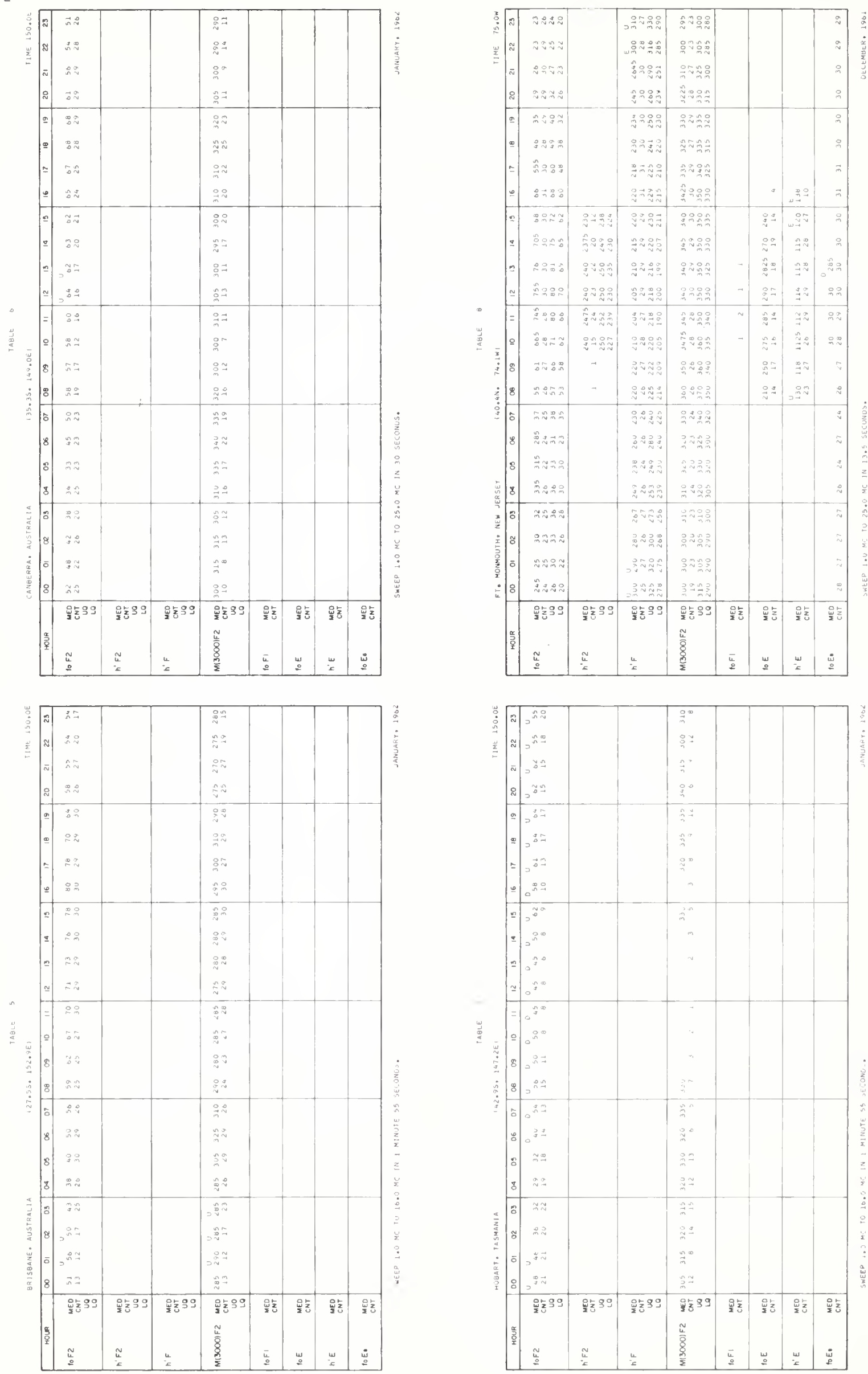

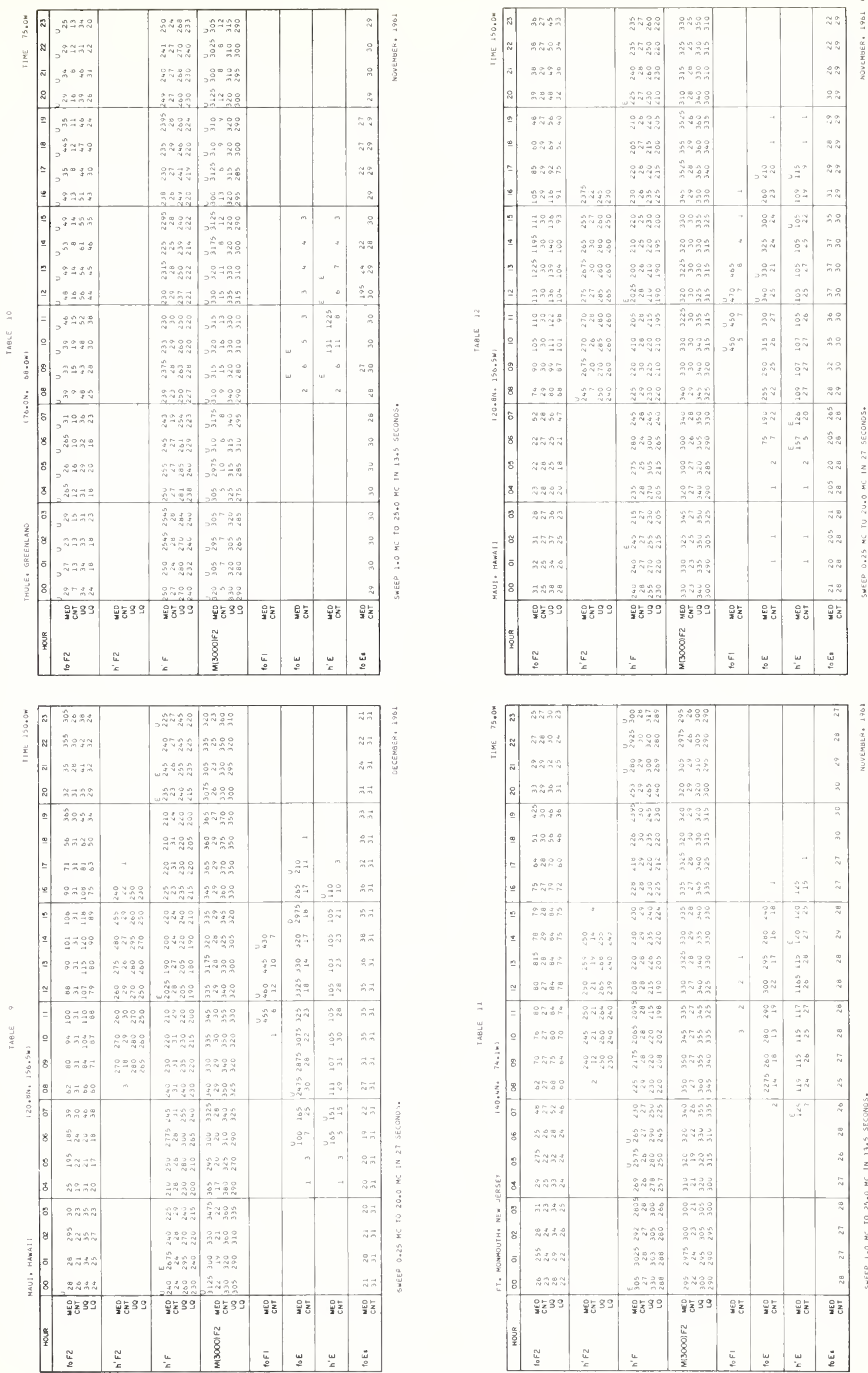

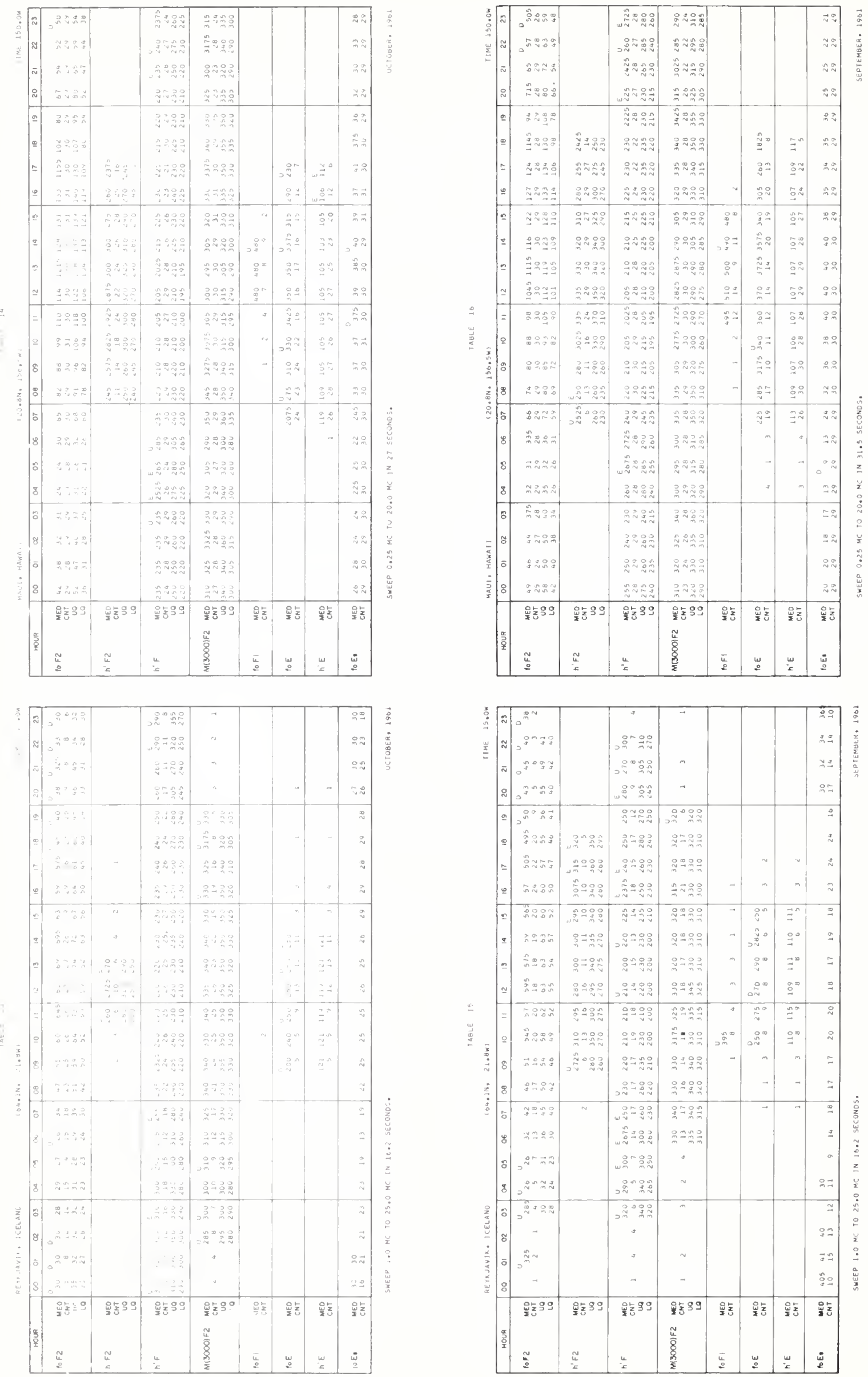

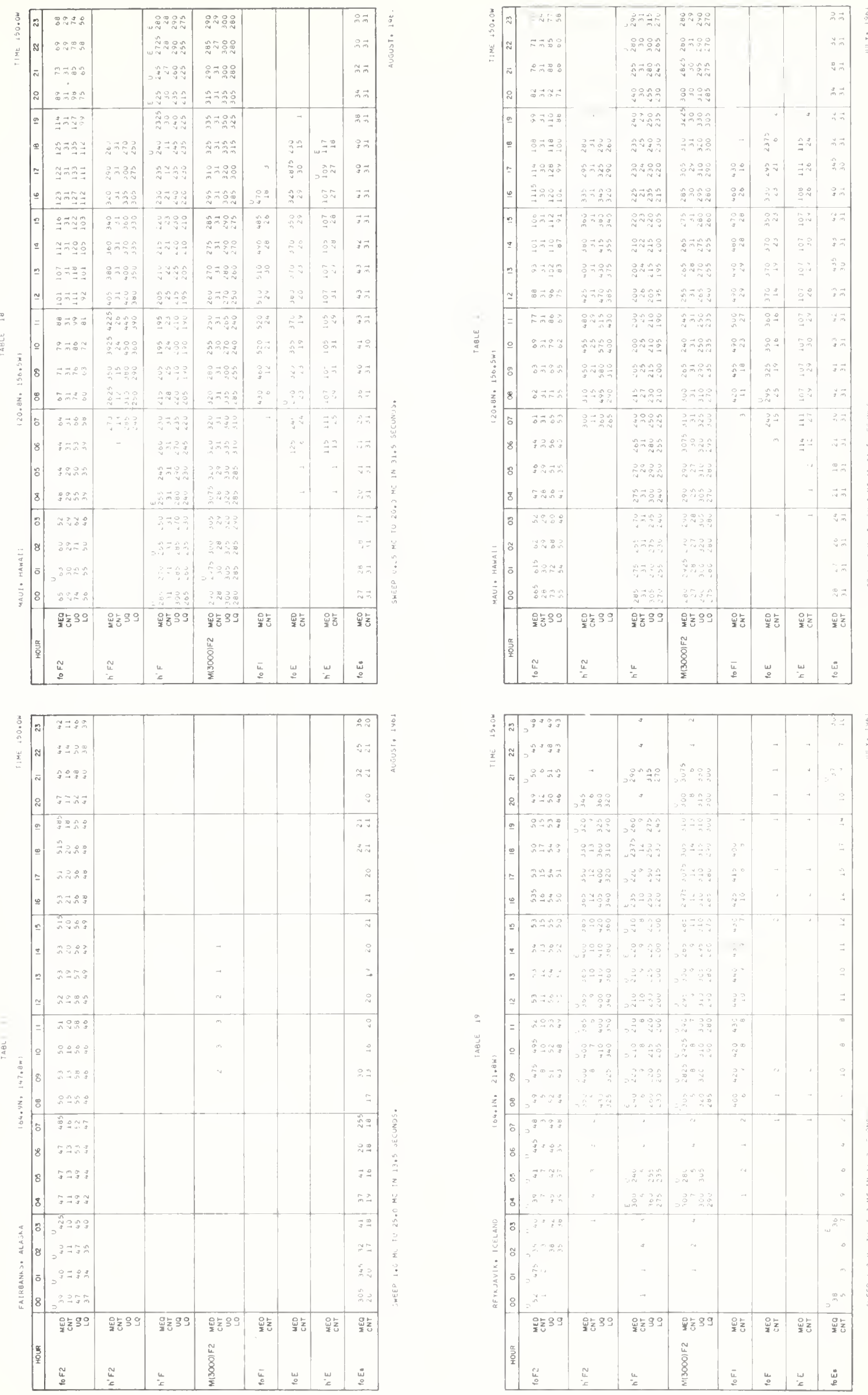


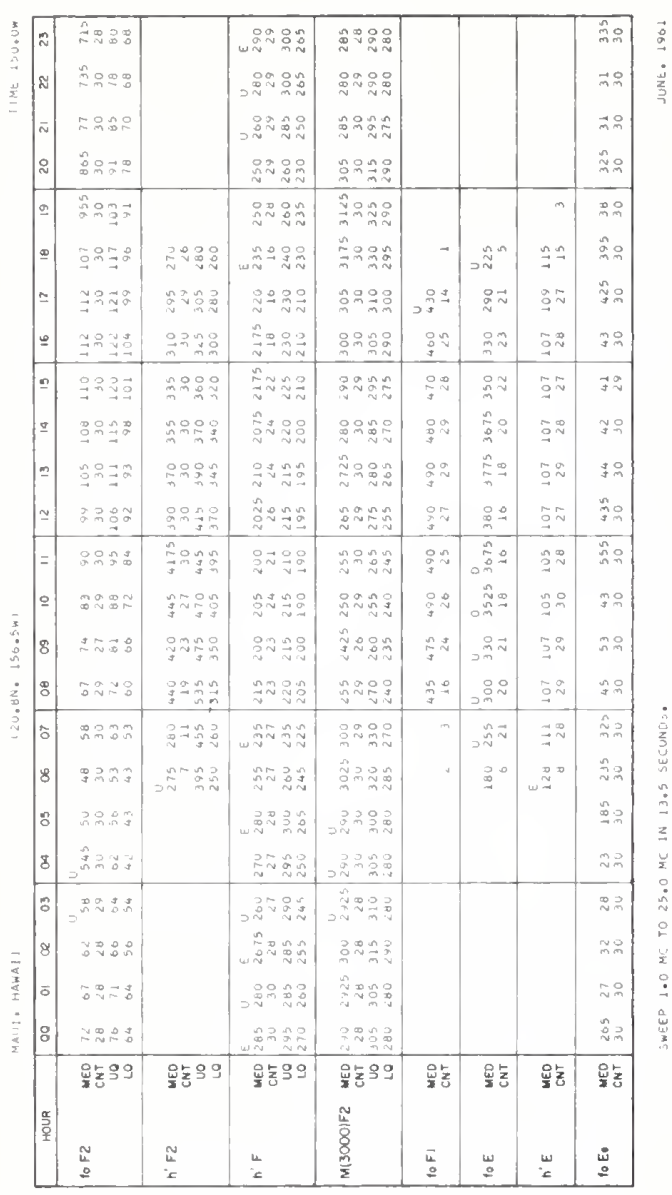

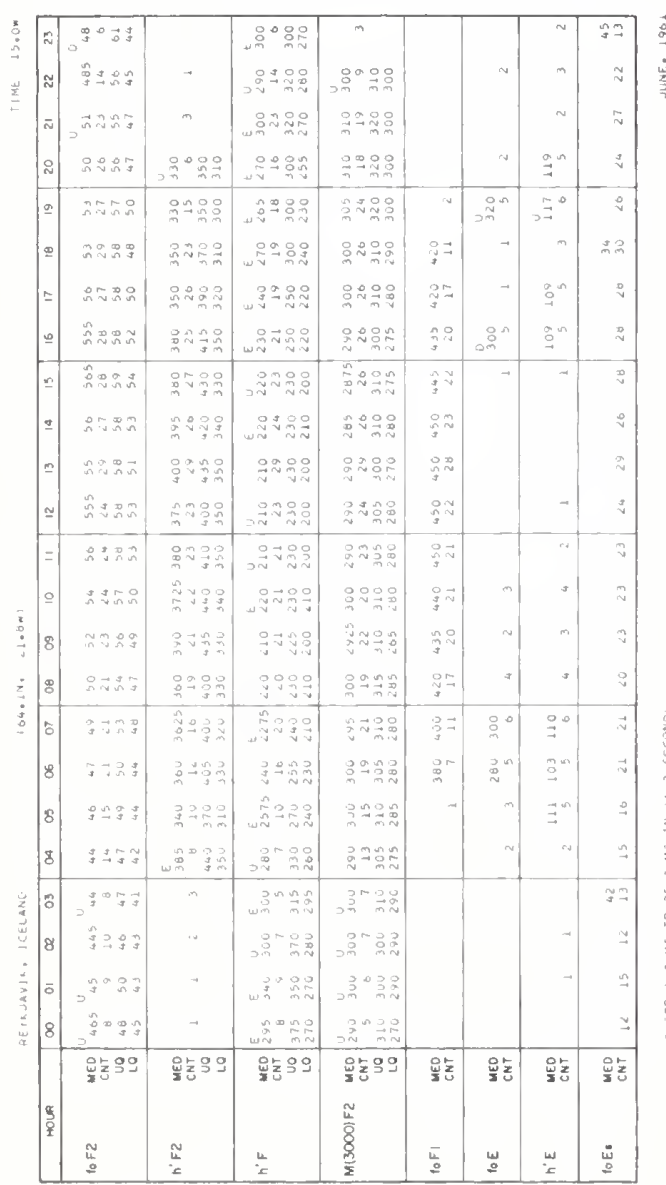

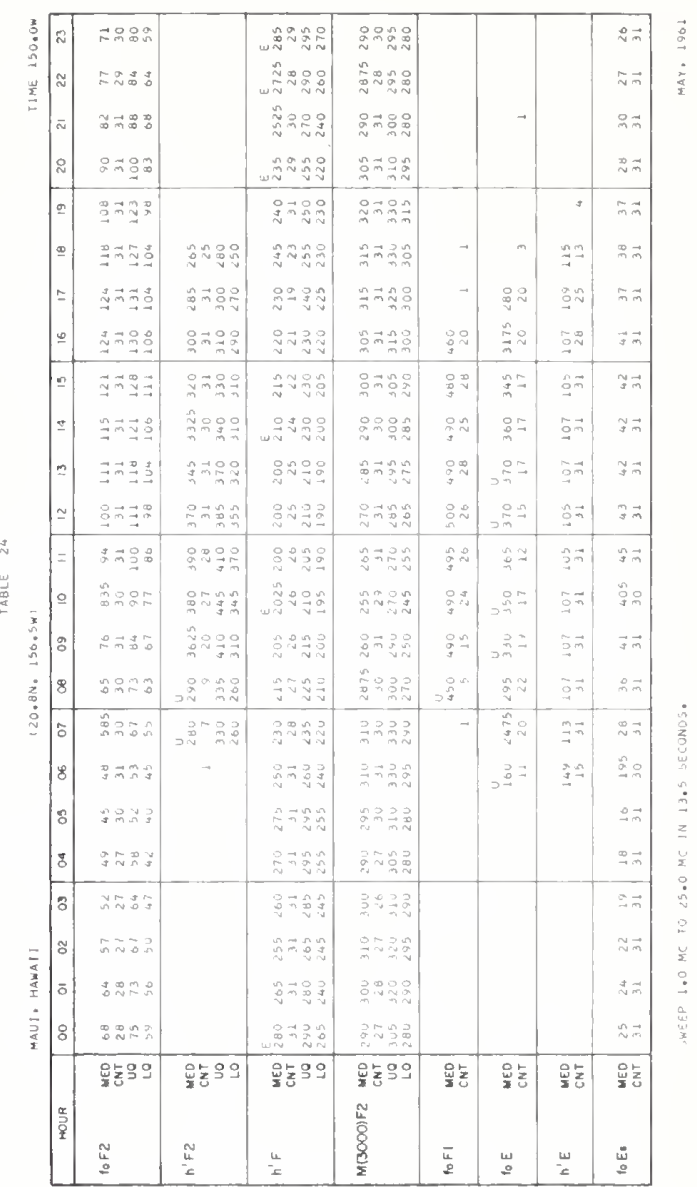

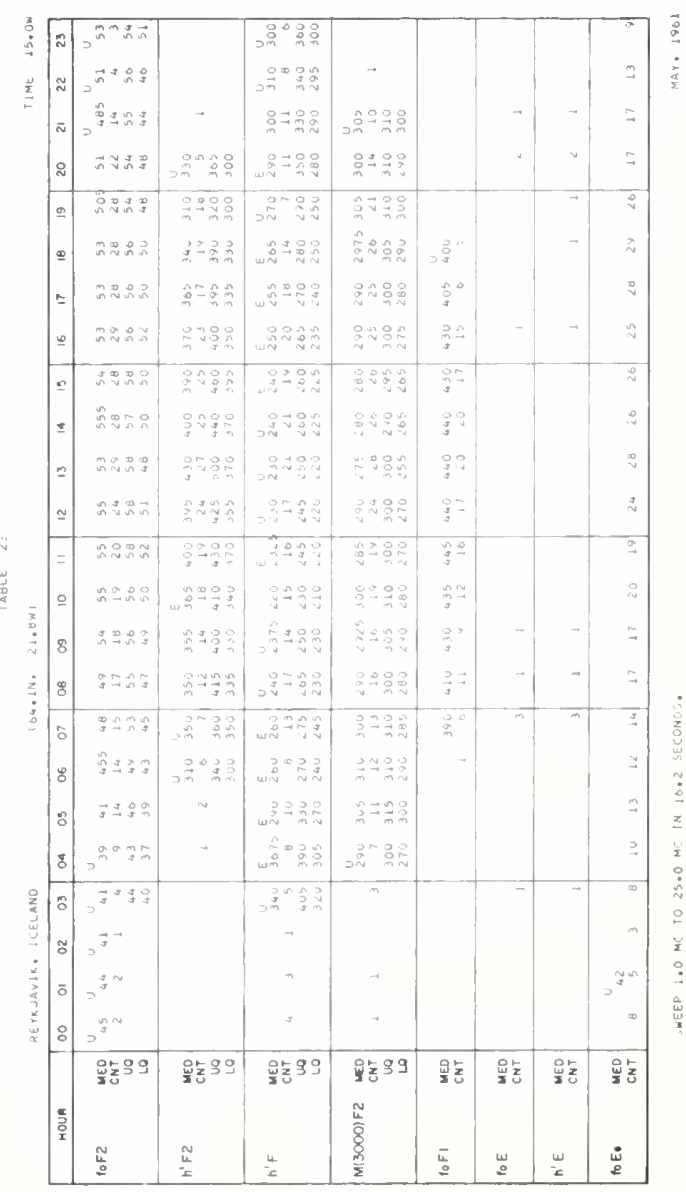



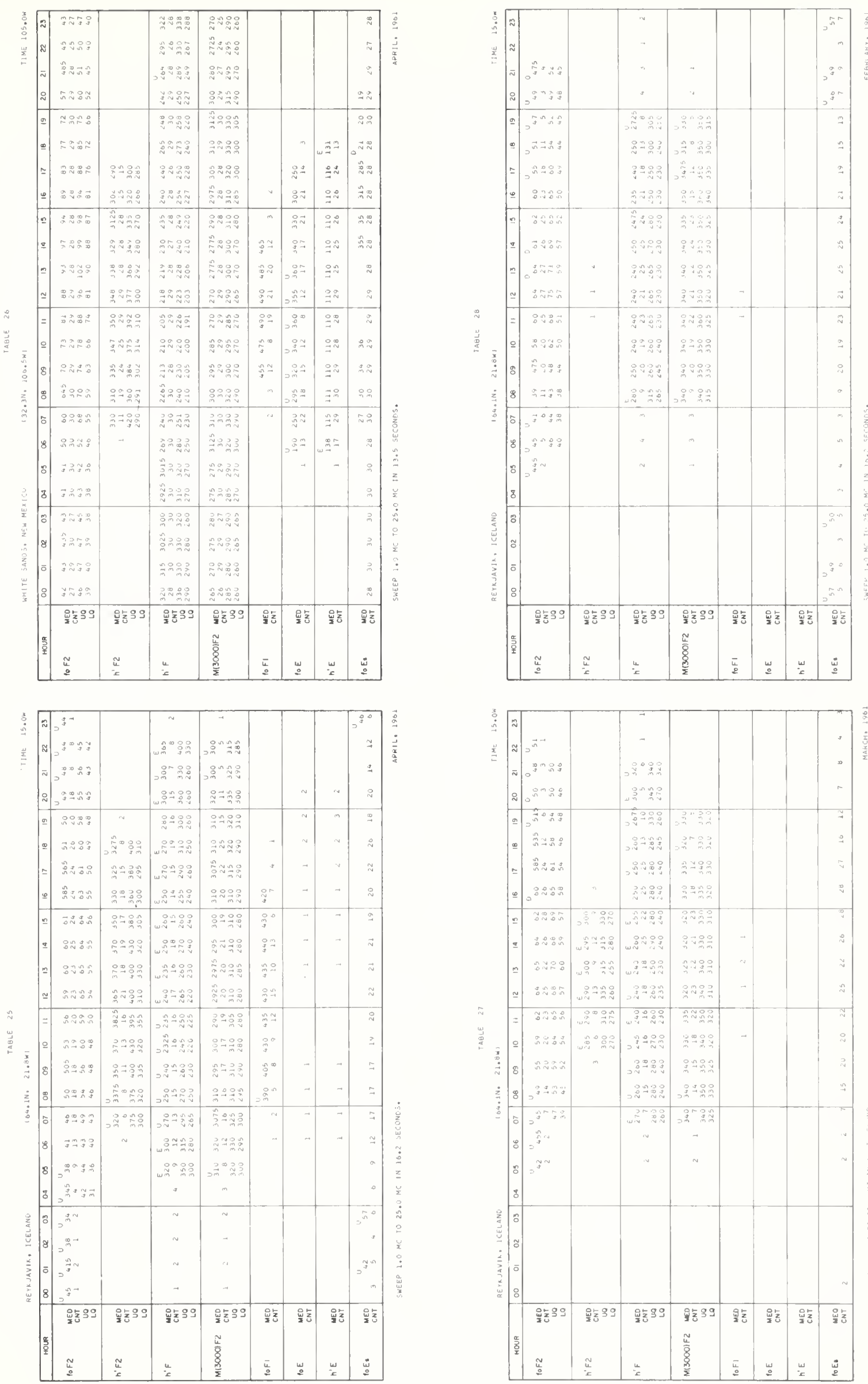

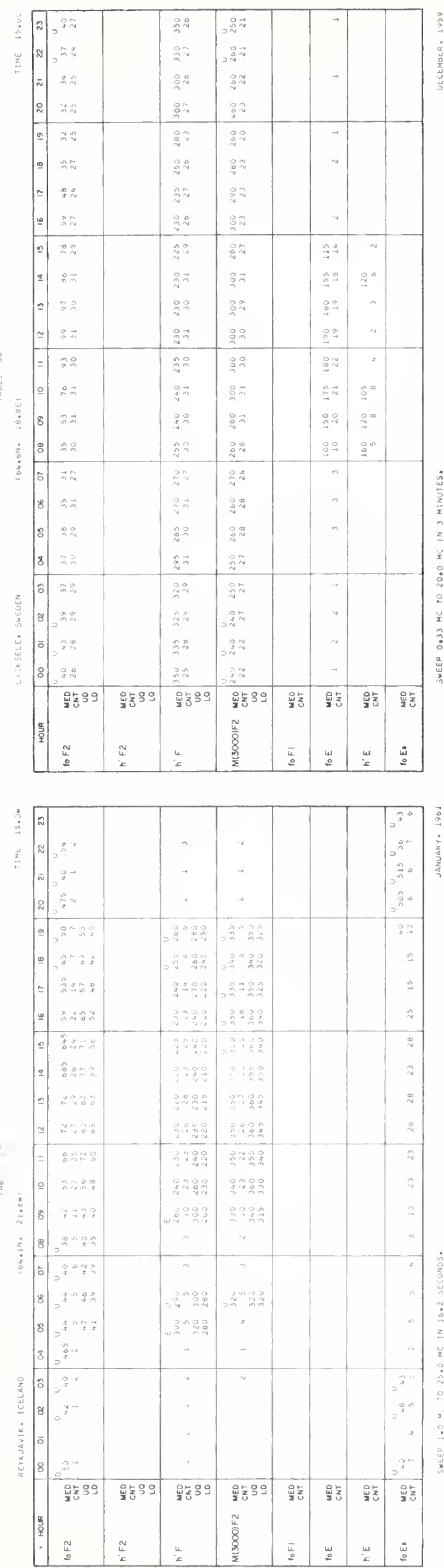
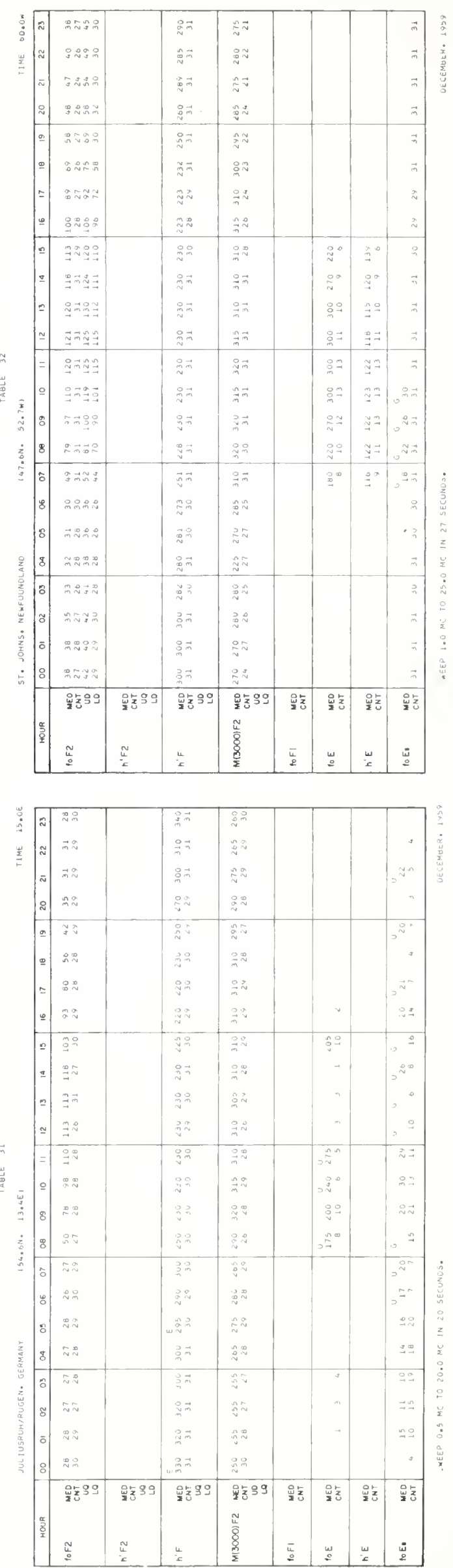

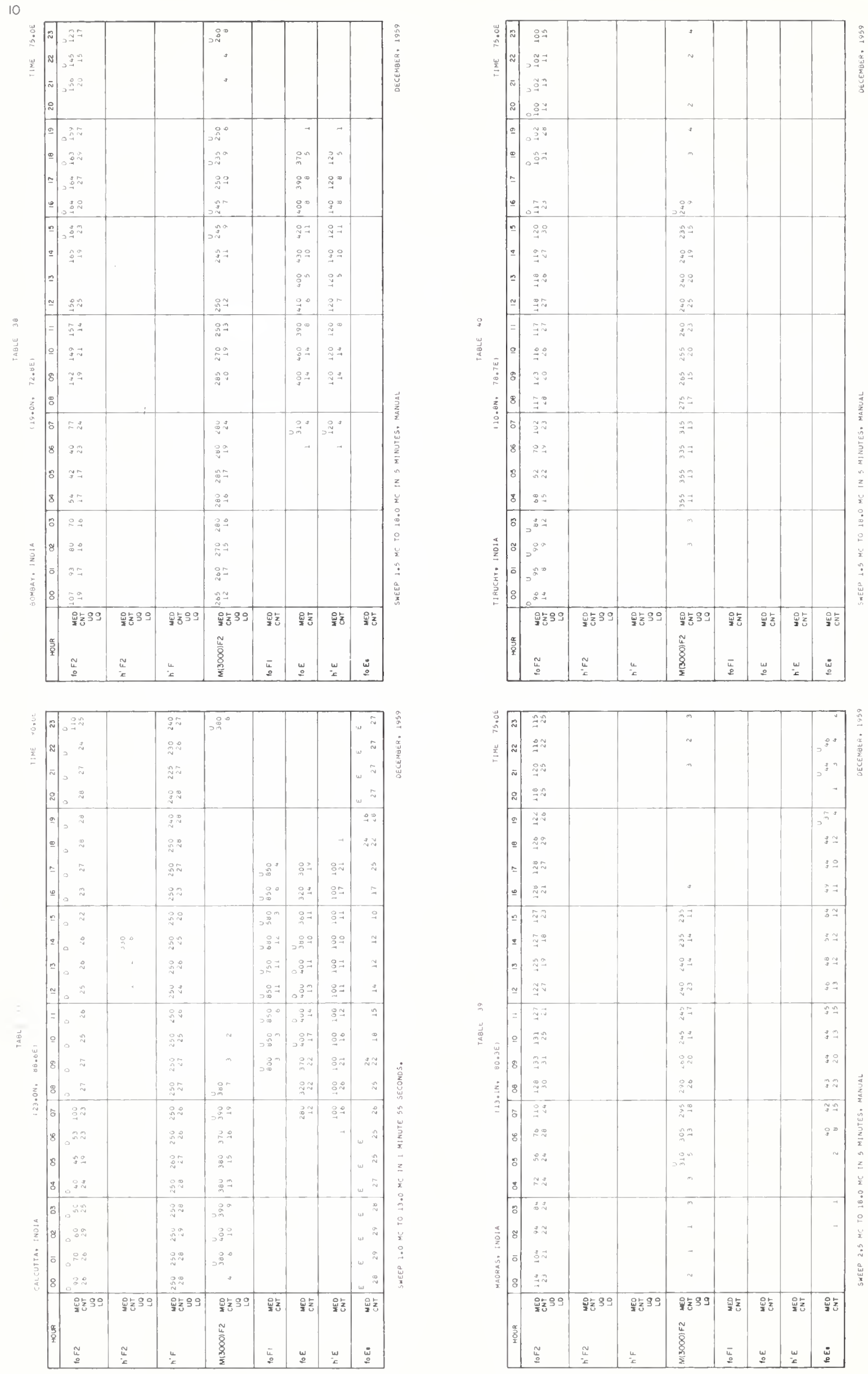

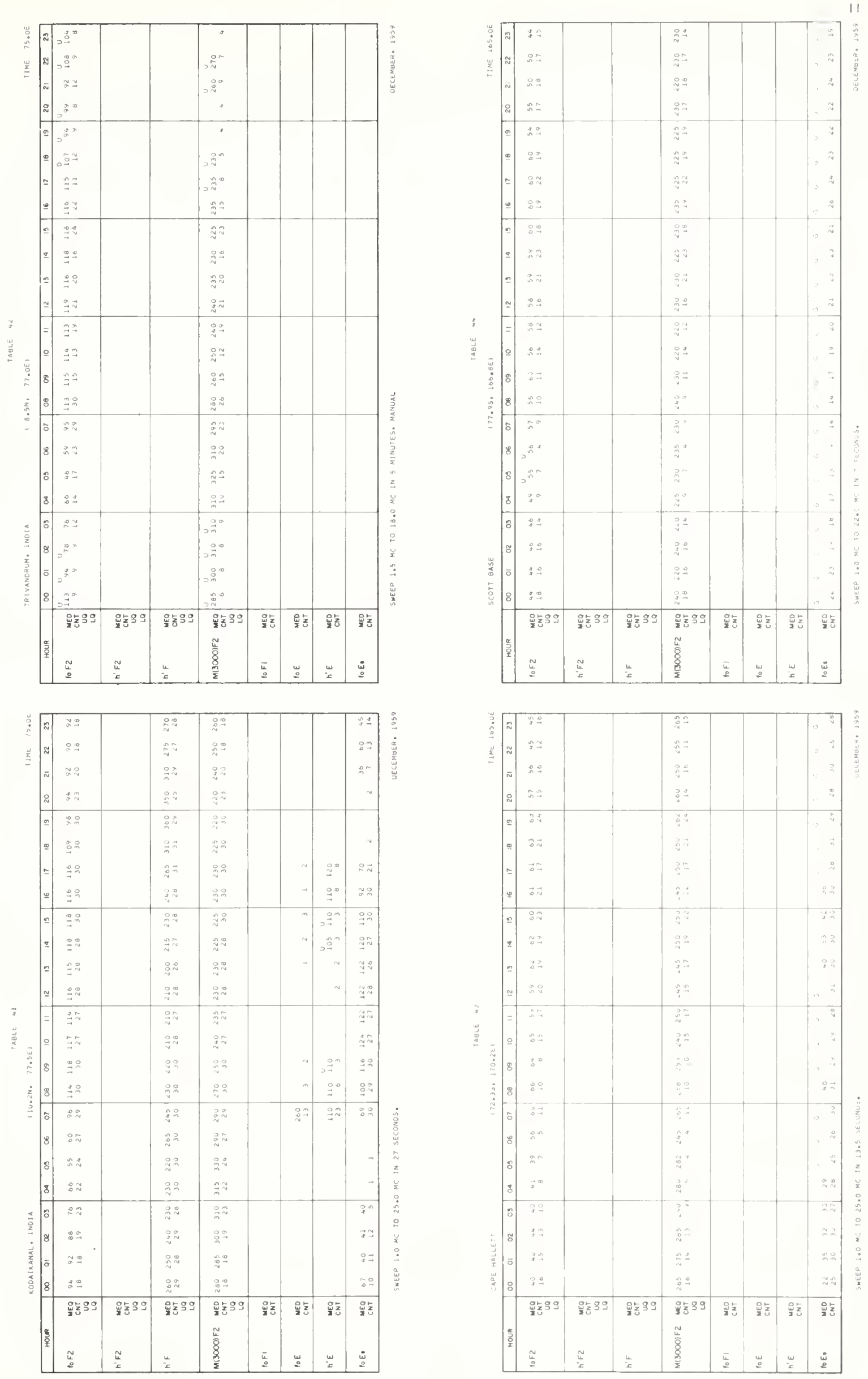

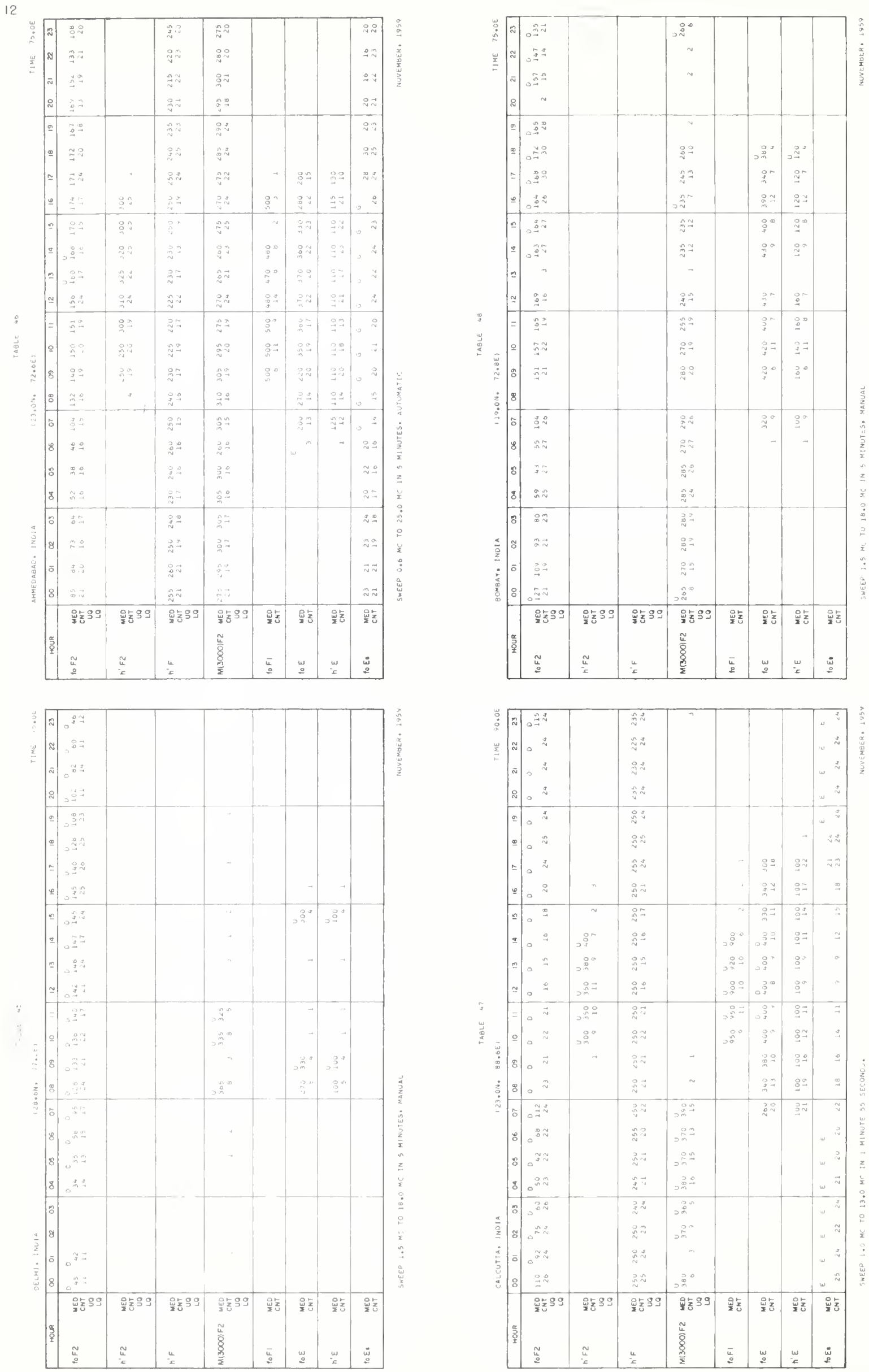

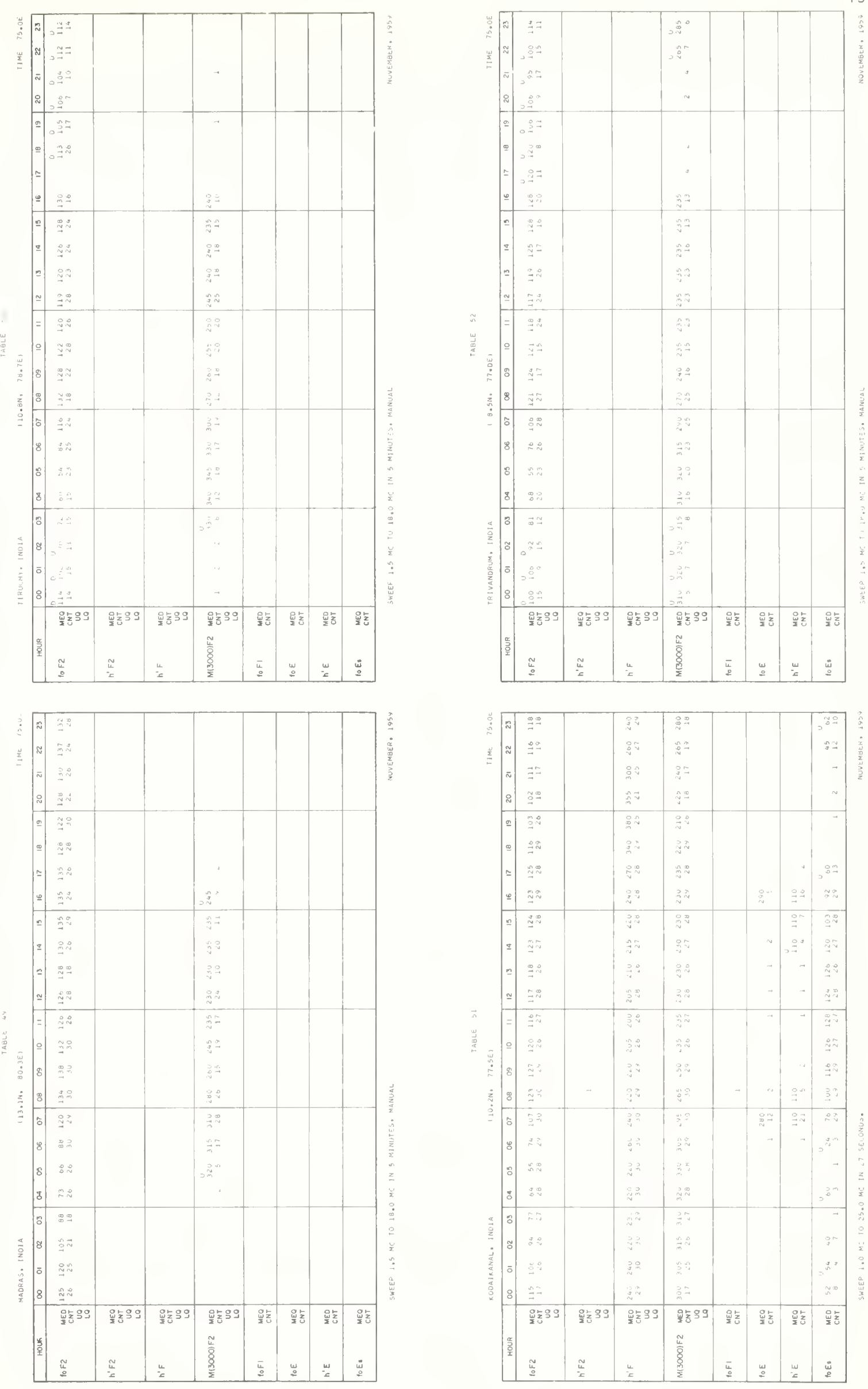

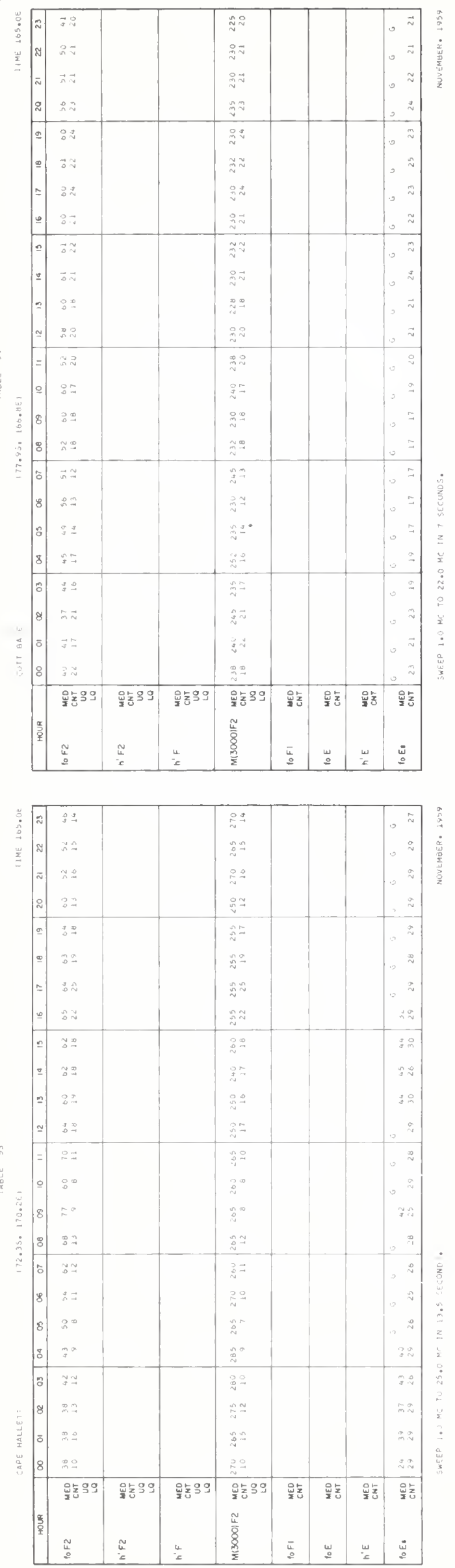
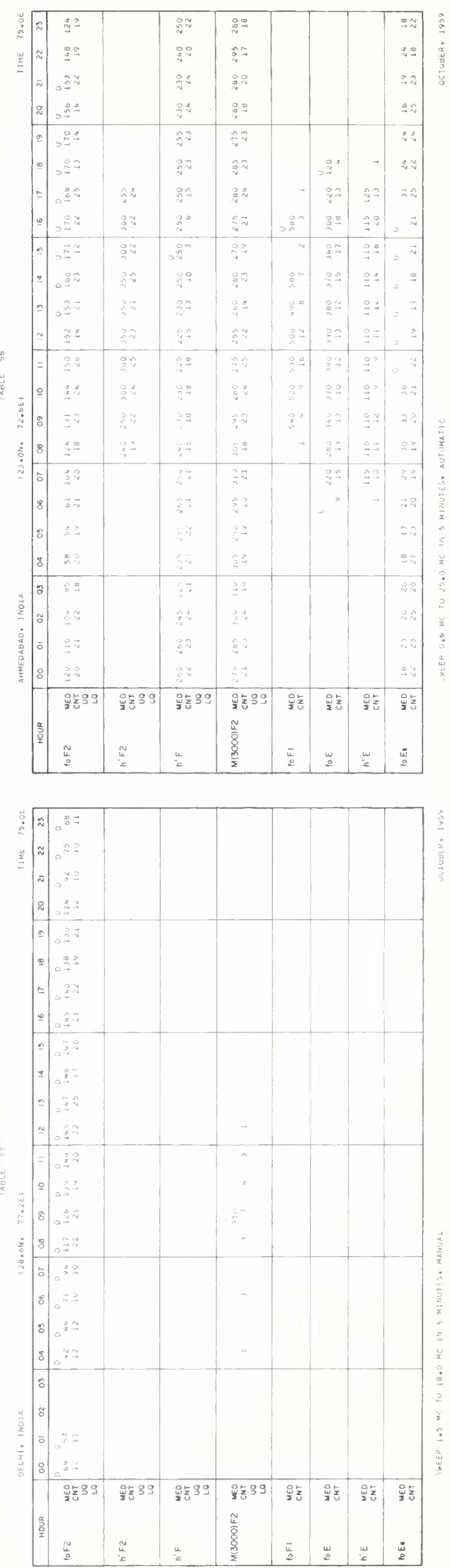

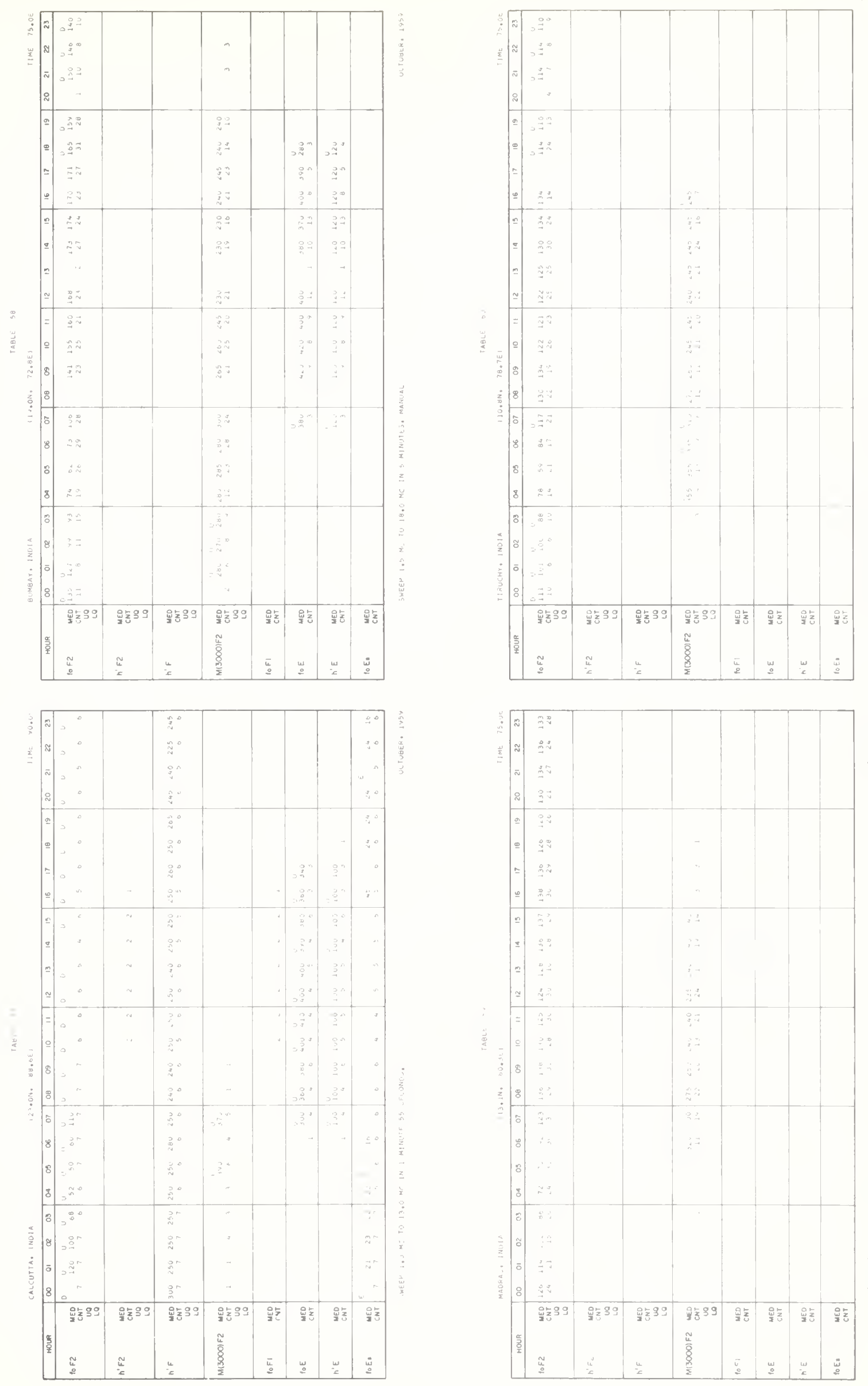

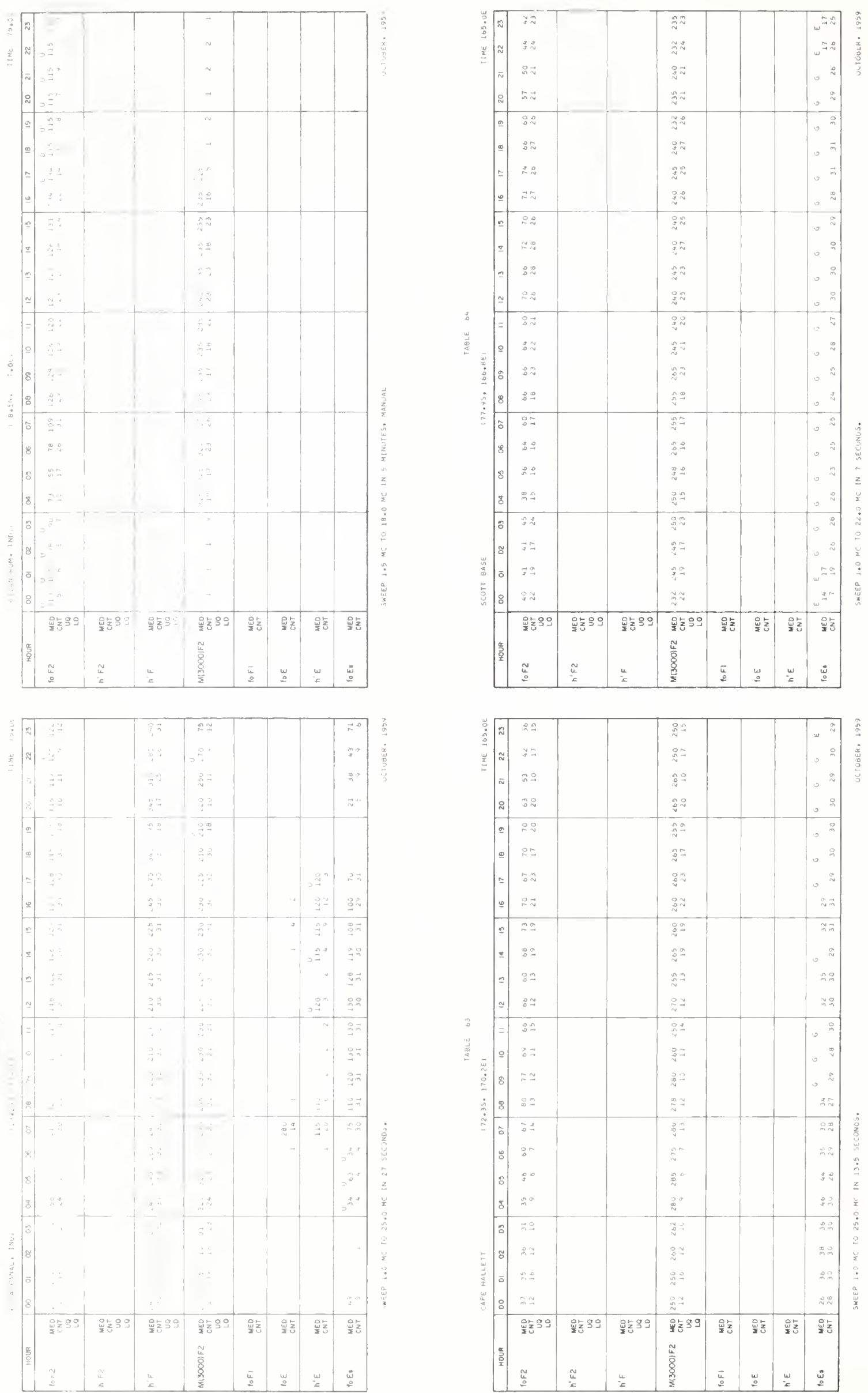

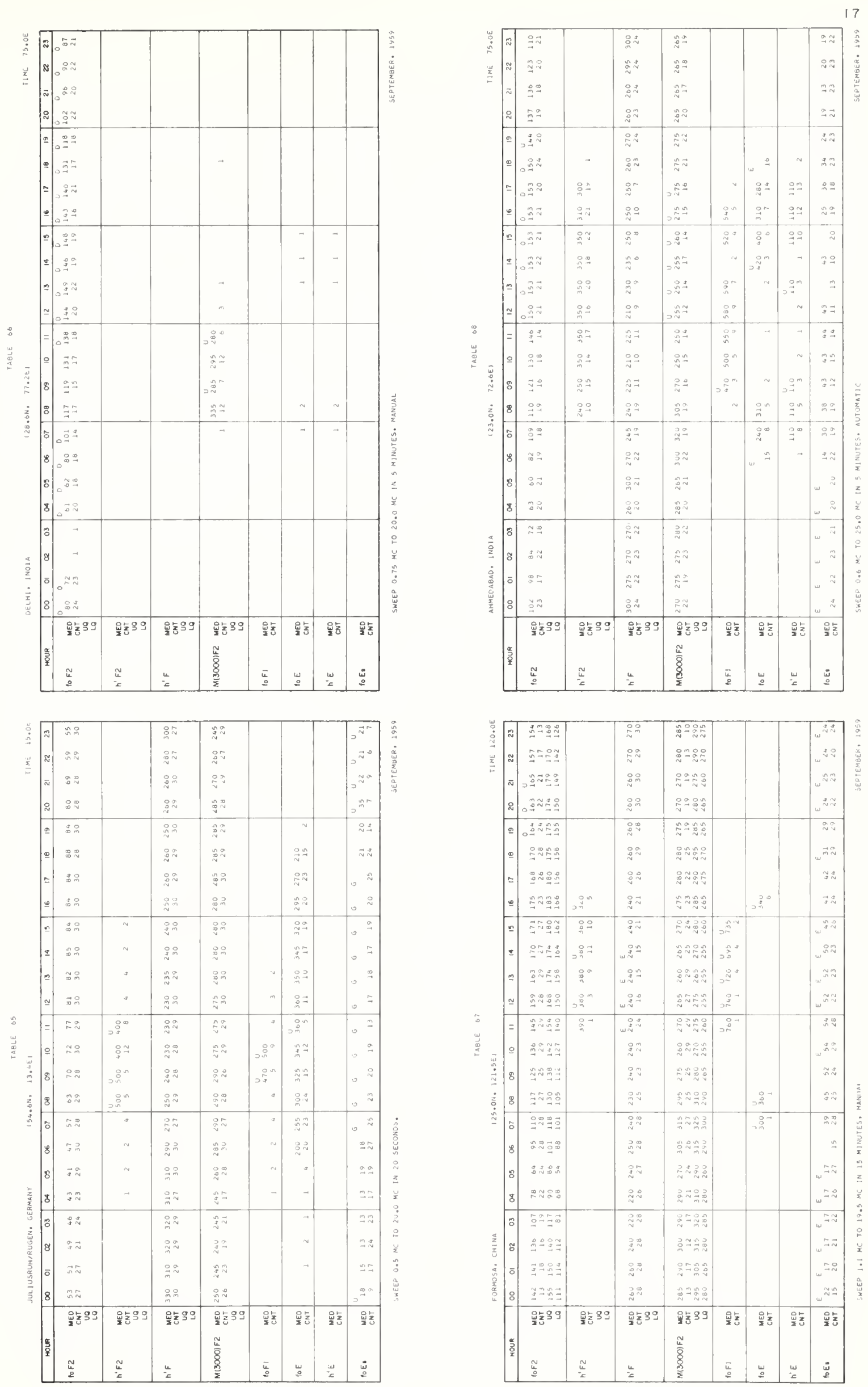

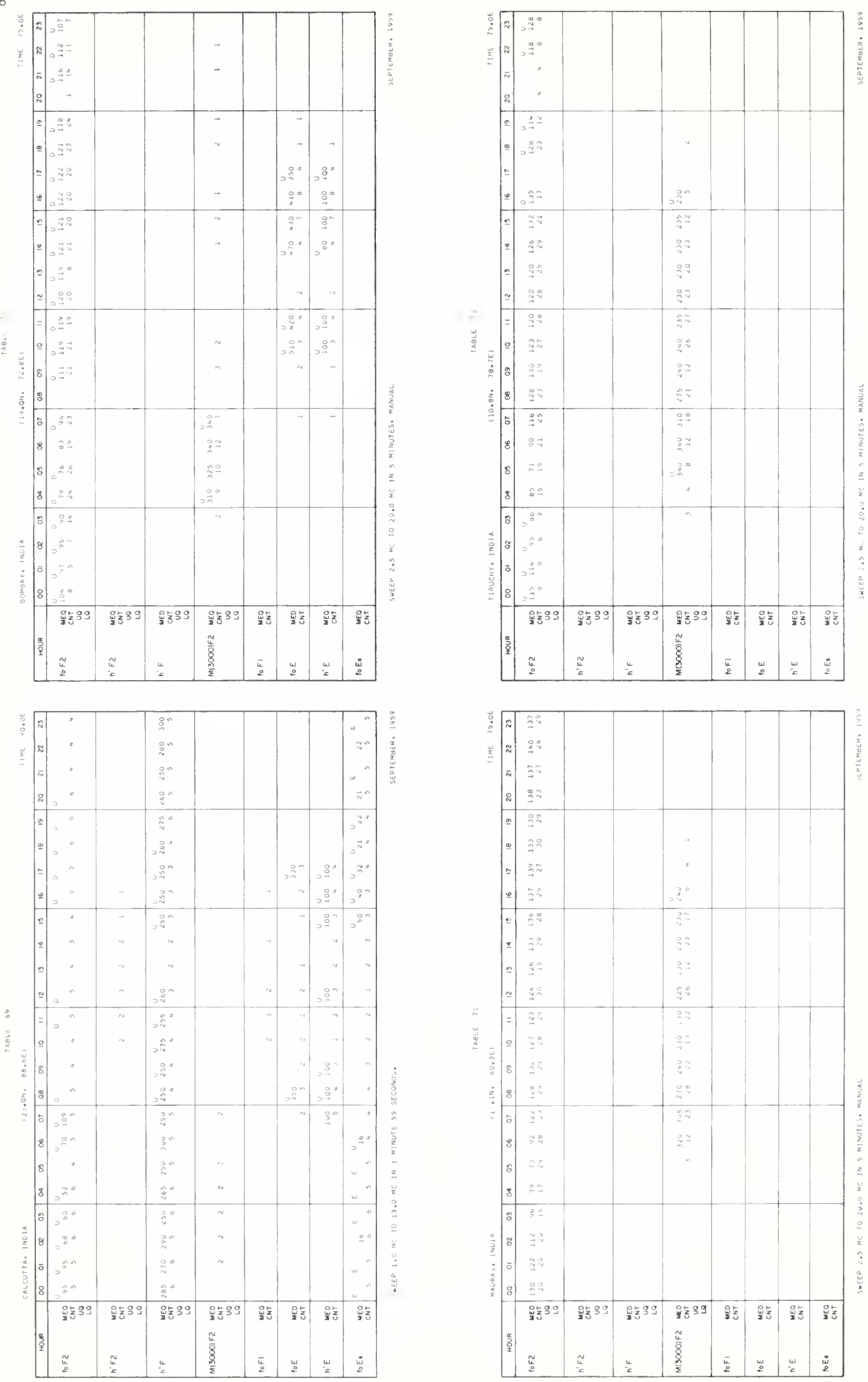

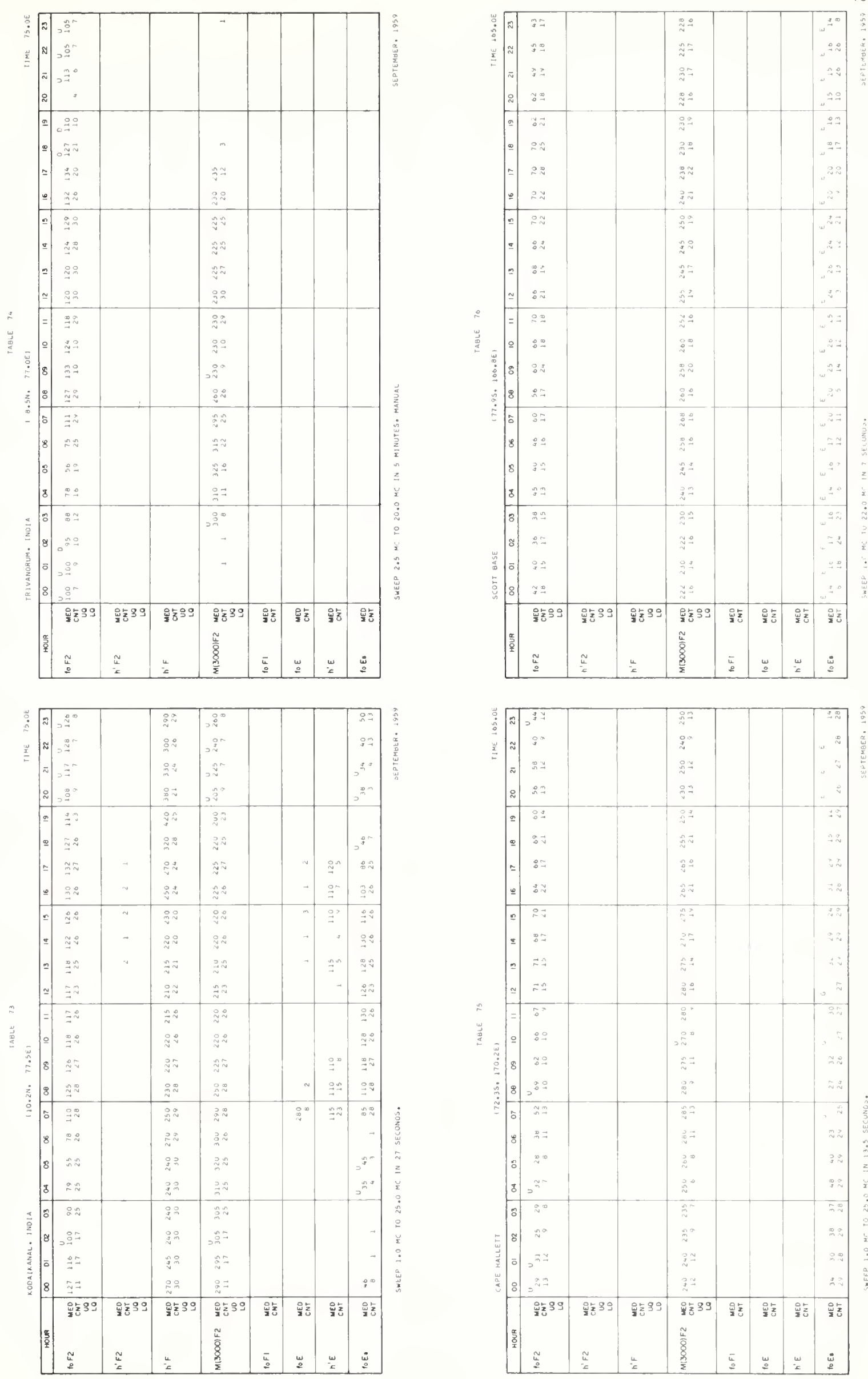

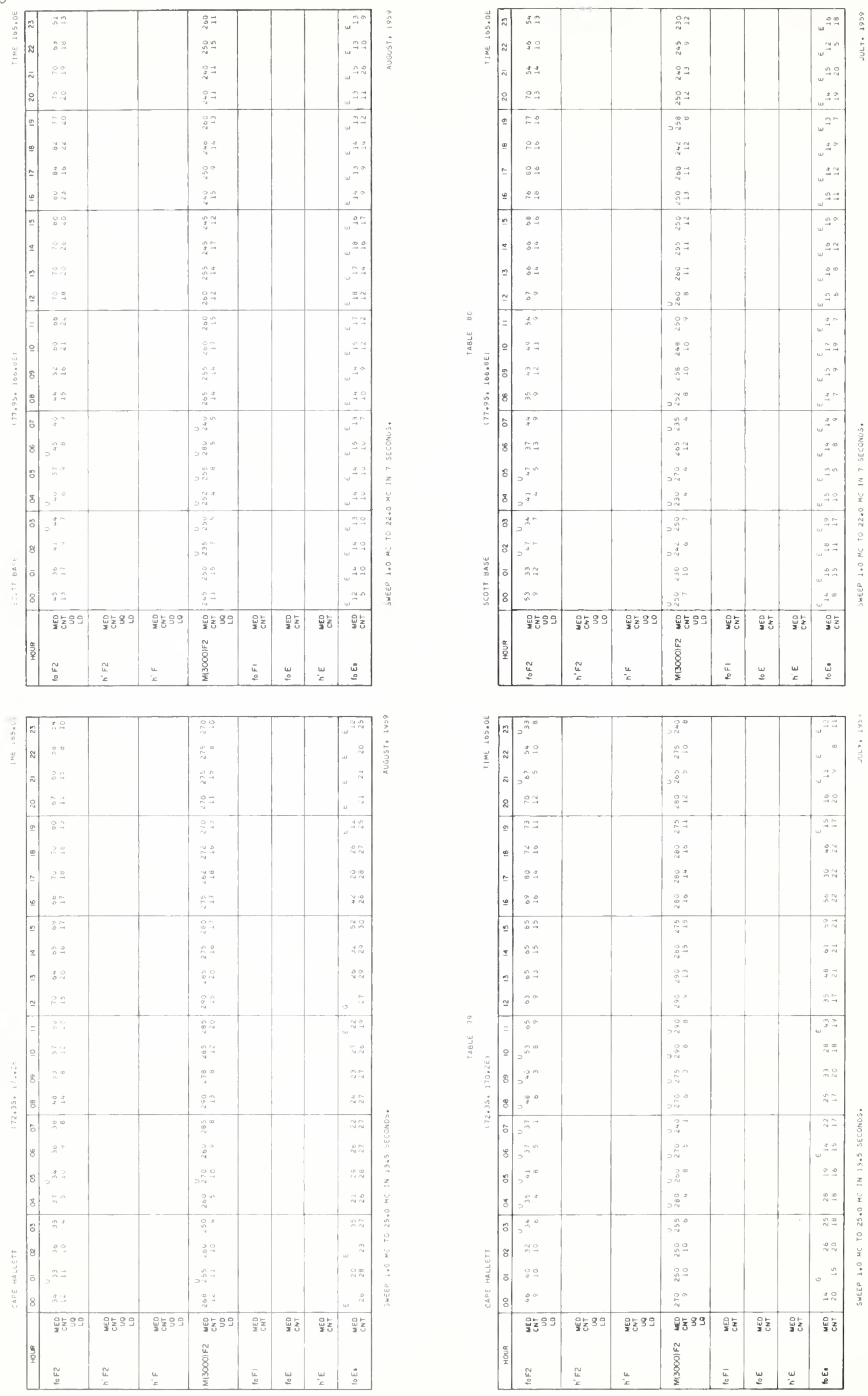

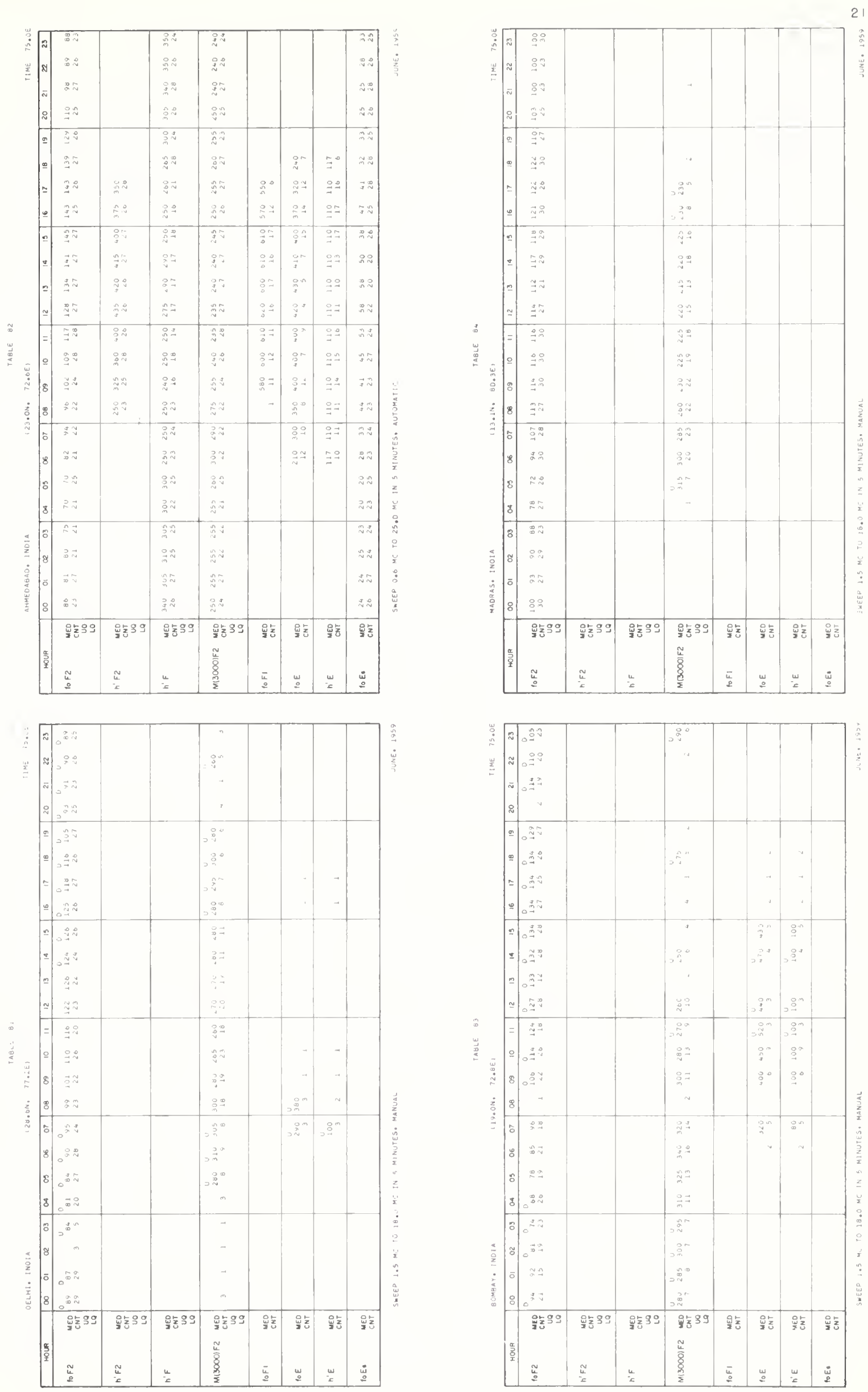
22
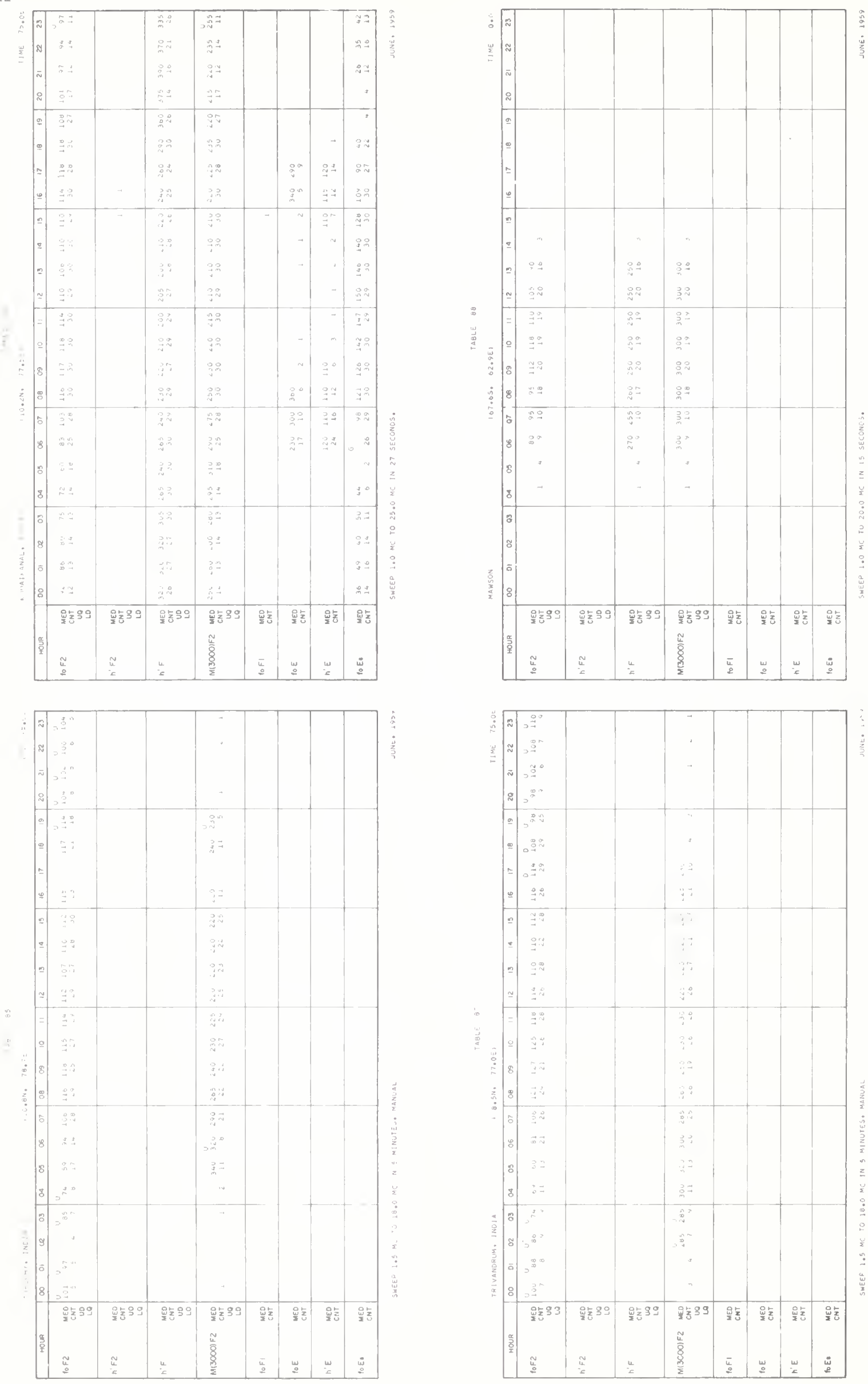

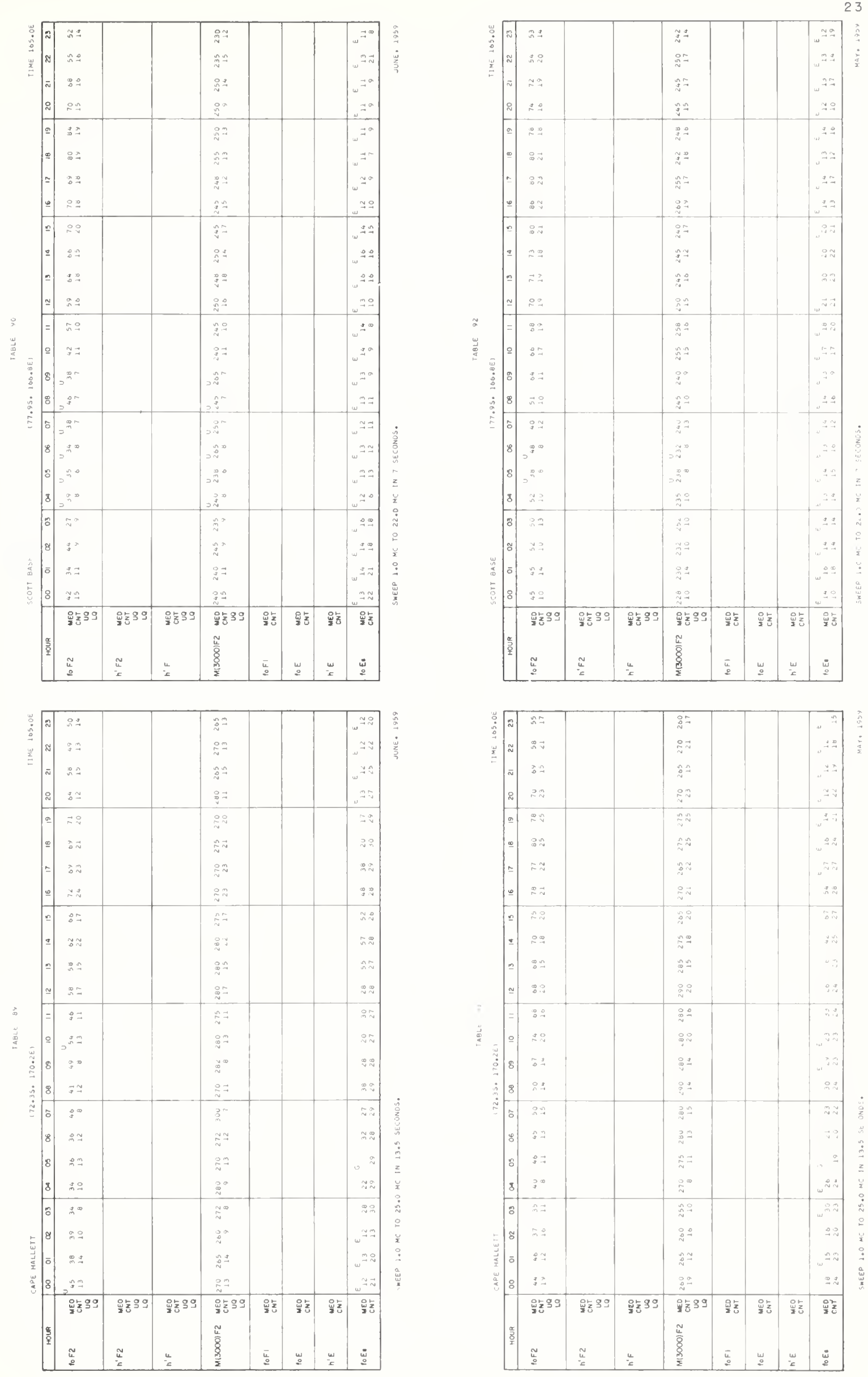

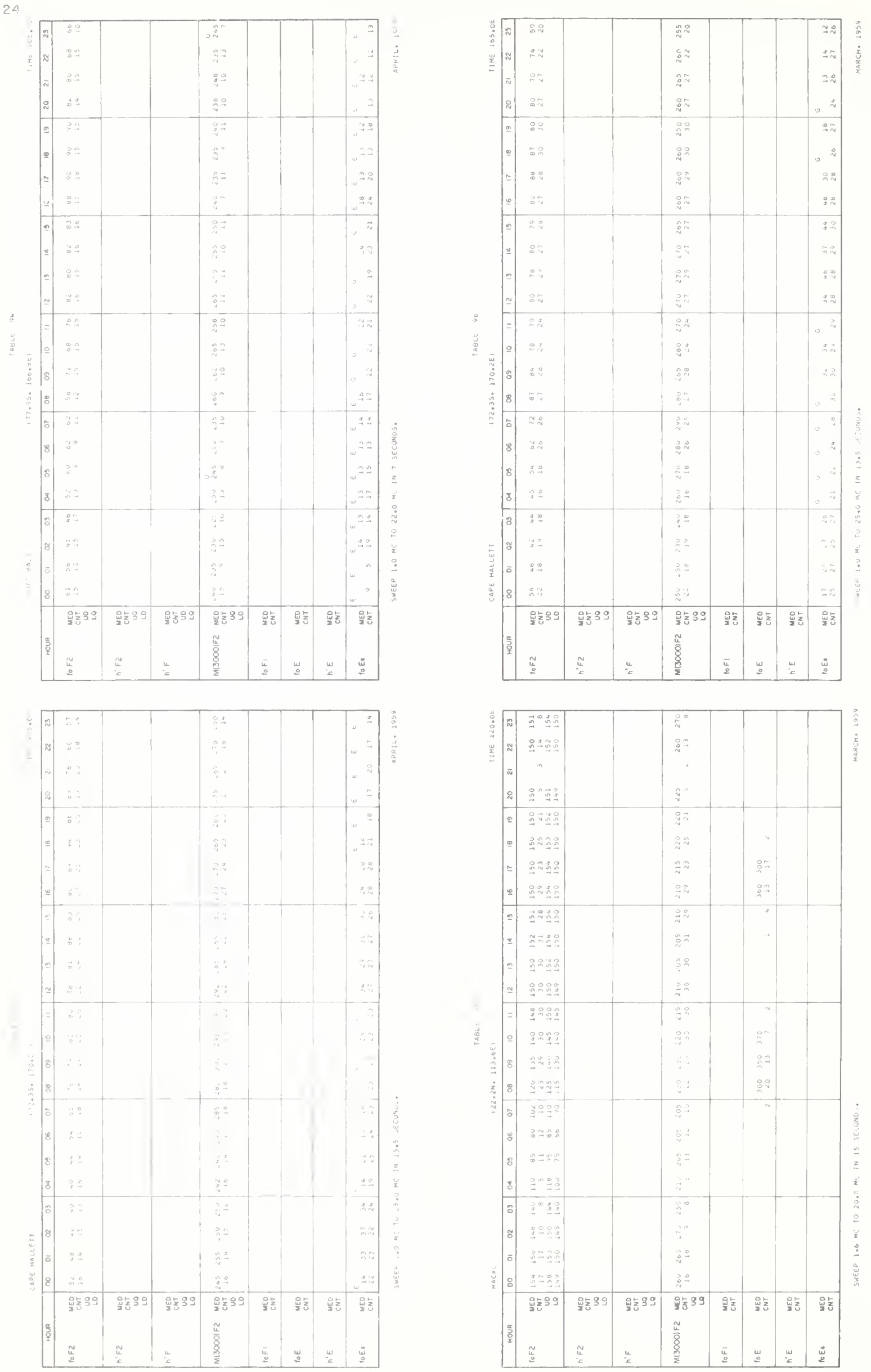

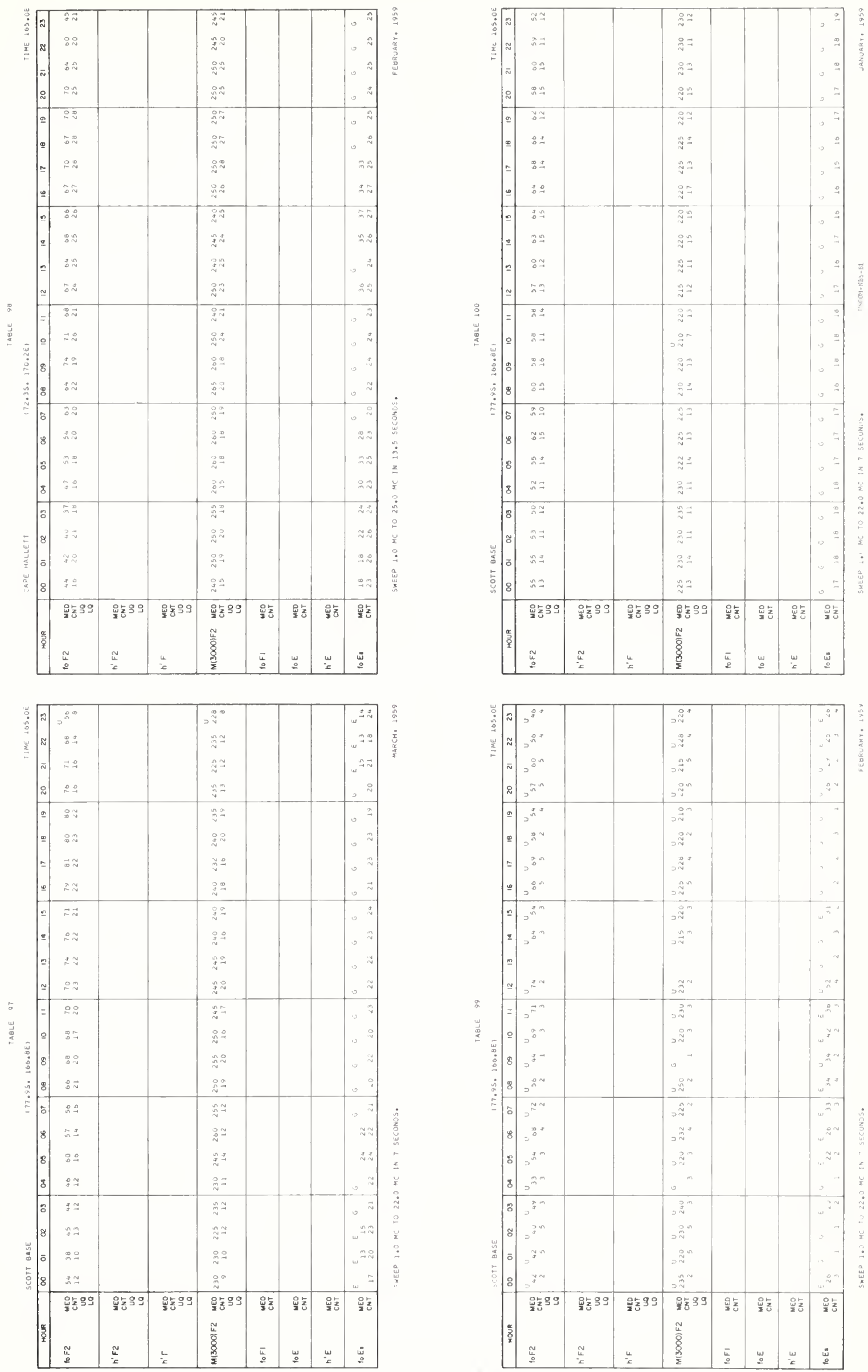


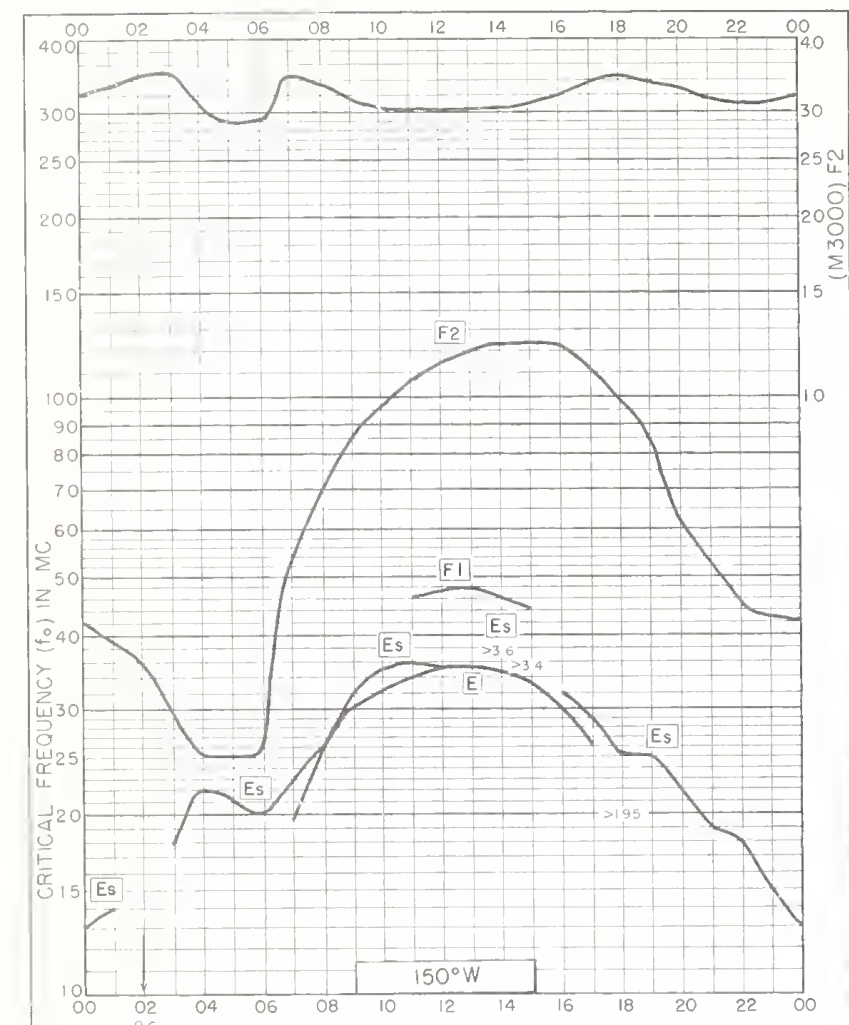

Fig. I. MAUI, HAWAII

$20.8^{\circ} \mathrm{N}, 156.5^{\circ} \mathrm{W}$

MARCH 1962

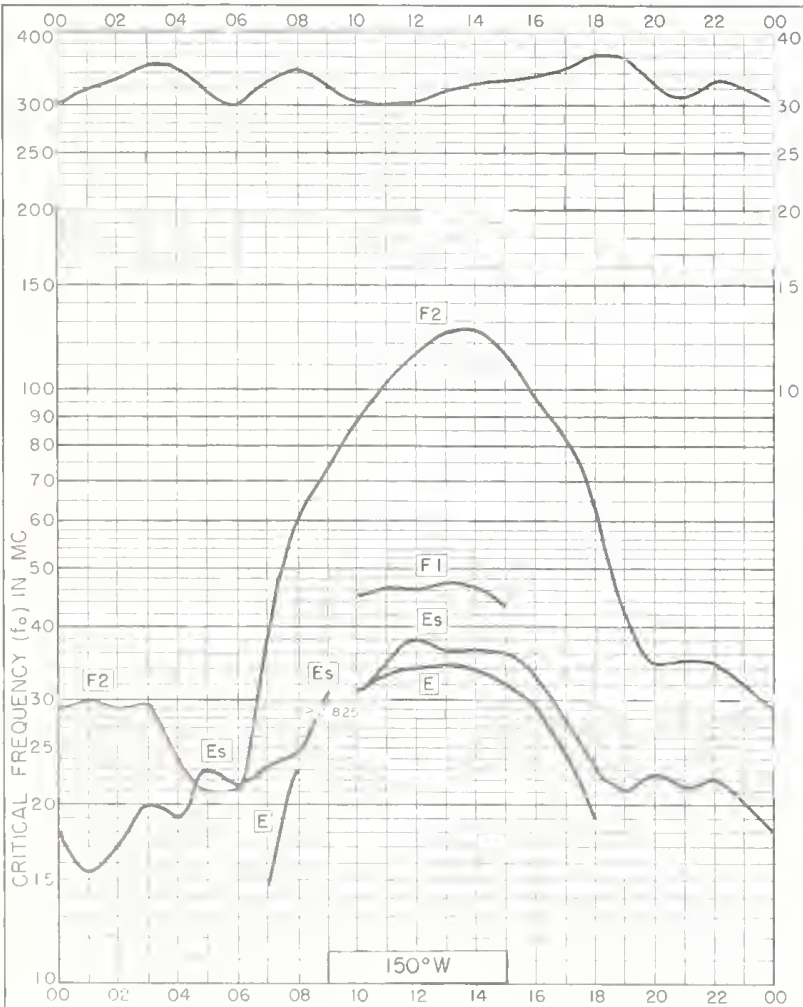

Fig. 3. MAUI, HAWAII

$20.8^{\circ} \mathrm{N}, 156.5^{\circ} \mathrm{W}$ FEBRUARY 1962

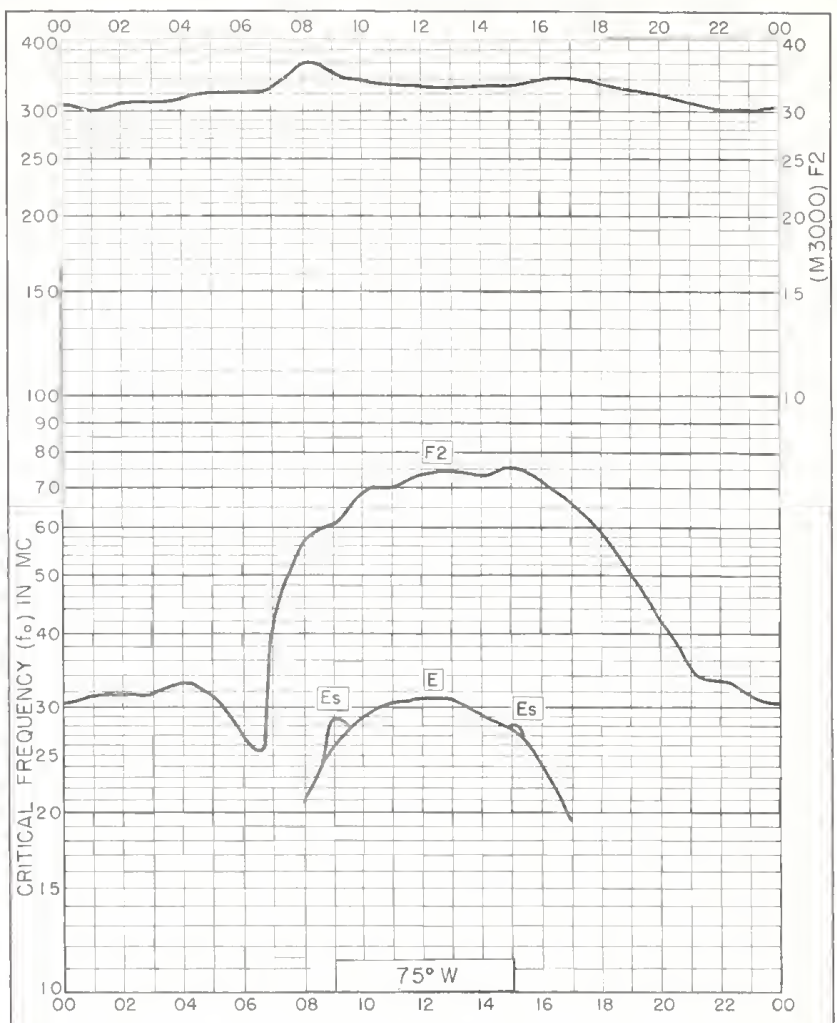

FIg 2. WASHINGTON, D.C $38.7^{\circ} \mathrm{N}, 77.1^{\circ} \mathrm{W} \quad$ FEBRUARY 1962

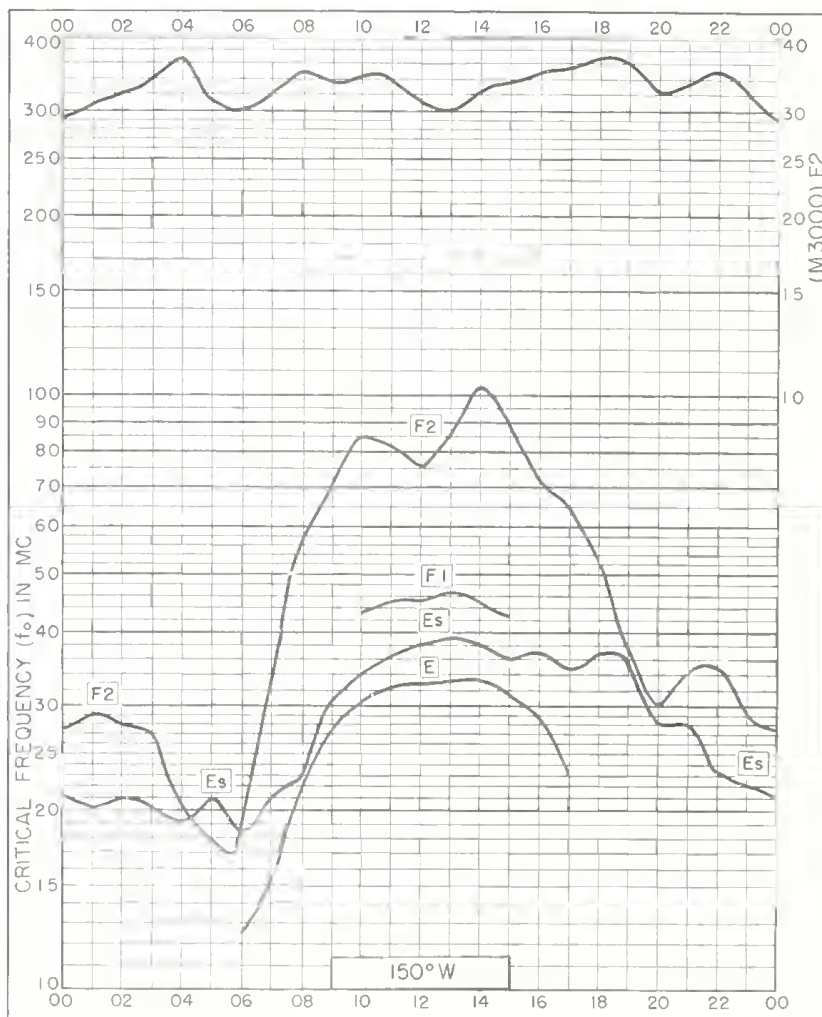

Fig. 4. MAUI, HAWAII

$20.8^{\circ} \mathrm{N}, 156.5^{\circ} \mathrm{W}$ JANUARY 1962 


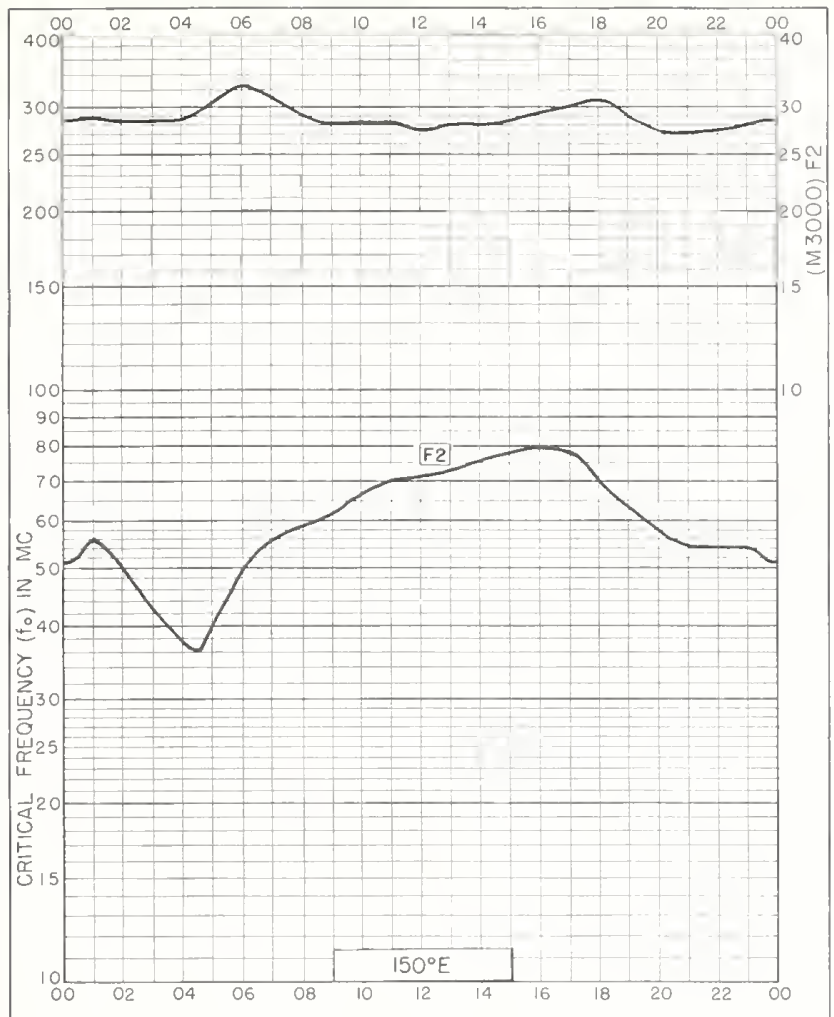

Fig. 5. BRISBANE, AUSTRALIA $27.5^{\circ} \mathrm{S}, 152.9^{\circ} \mathrm{E}$ JANUARY 1962

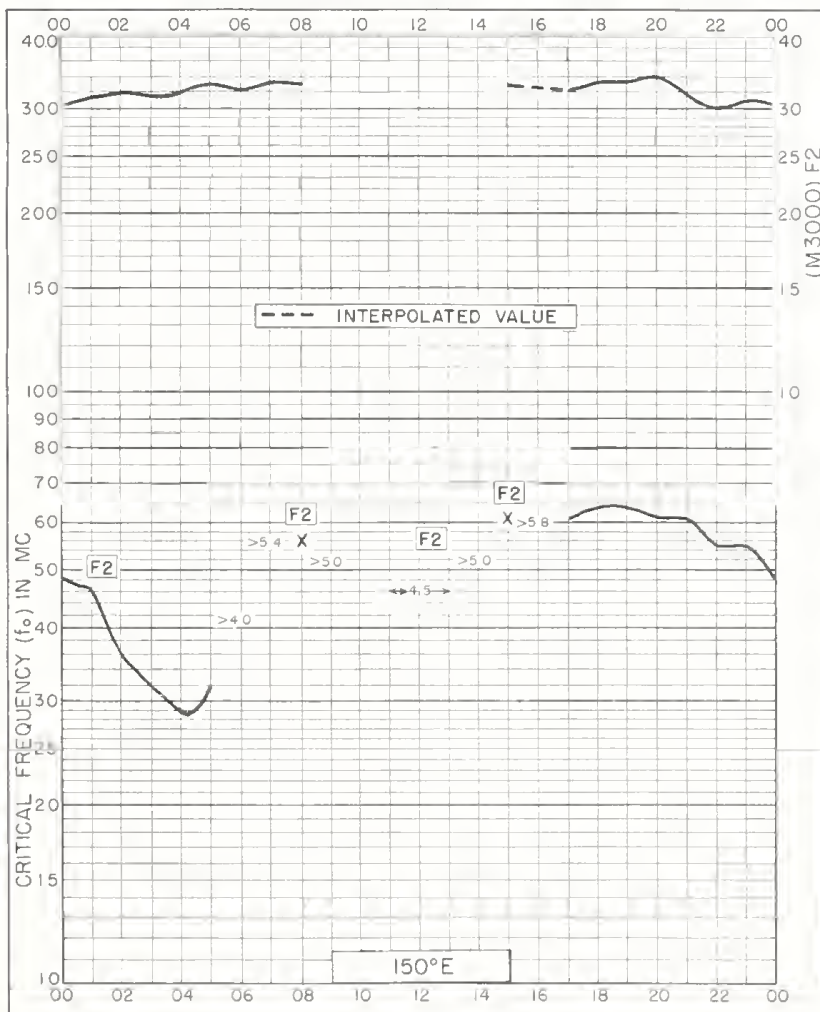

Fig. 7. HOBART, TASMANIA $42.9^{\circ} \mathrm{S}, 147.2^{\circ} \mathrm{E}$ JANUARY 1962
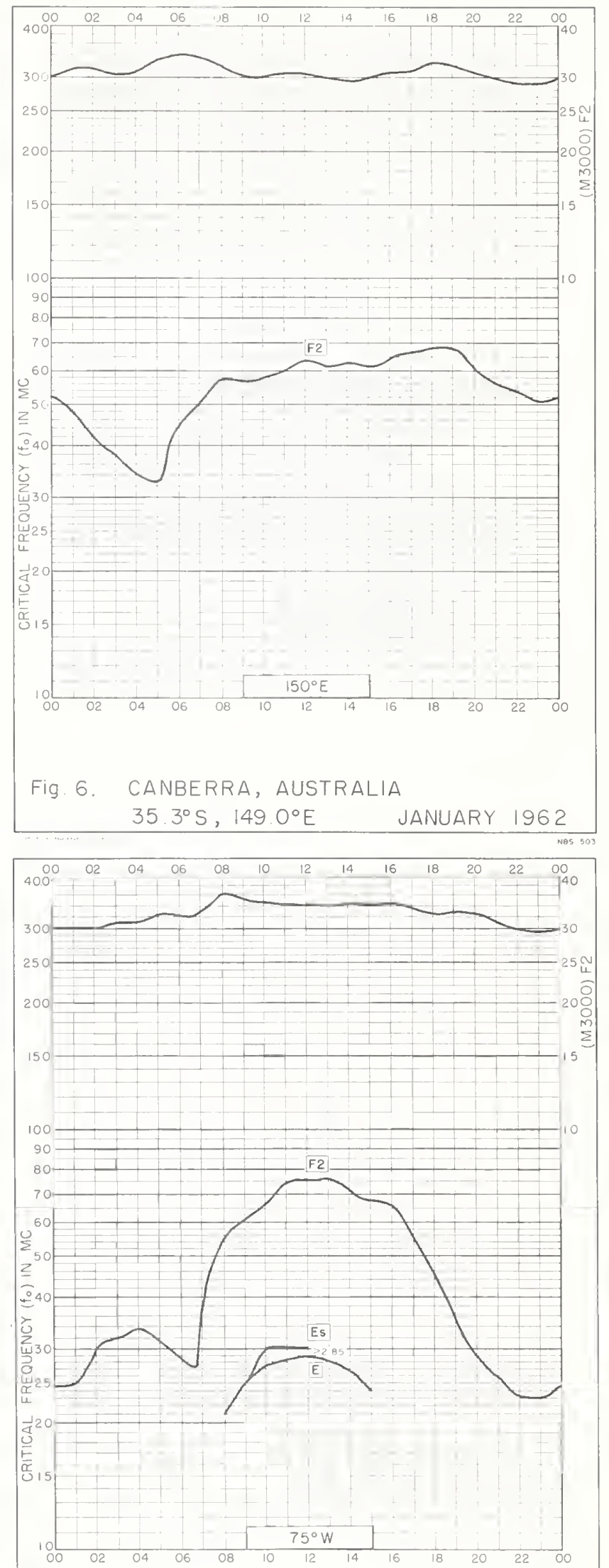

Fig. 8. FT. MONMOUTH, NEW JERSEY $40.4^{\circ} \mathrm{N}, 74.1^{\circ} \mathrm{W}$ DECEMBER $196 \mid$ 


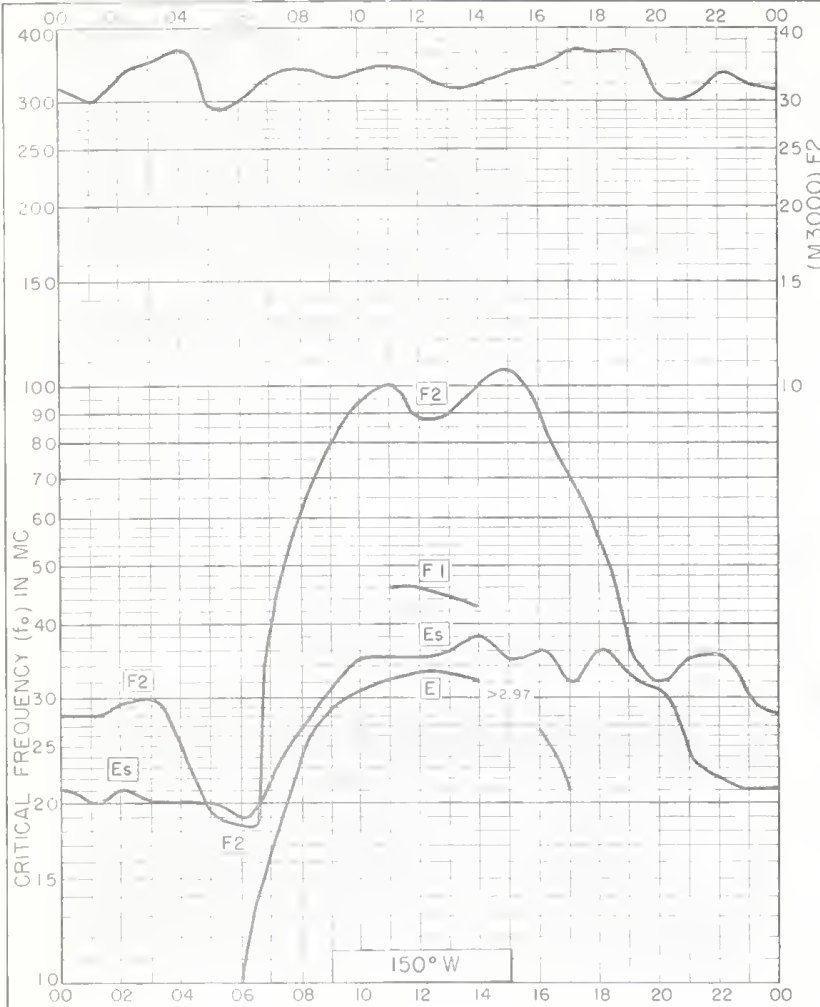

Fig. 9. MAUL, HAWAII

$20.8^{\circ} \mathrm{N}, 156.5^{\circ} \mathrm{W}$ DECEMBER 1961

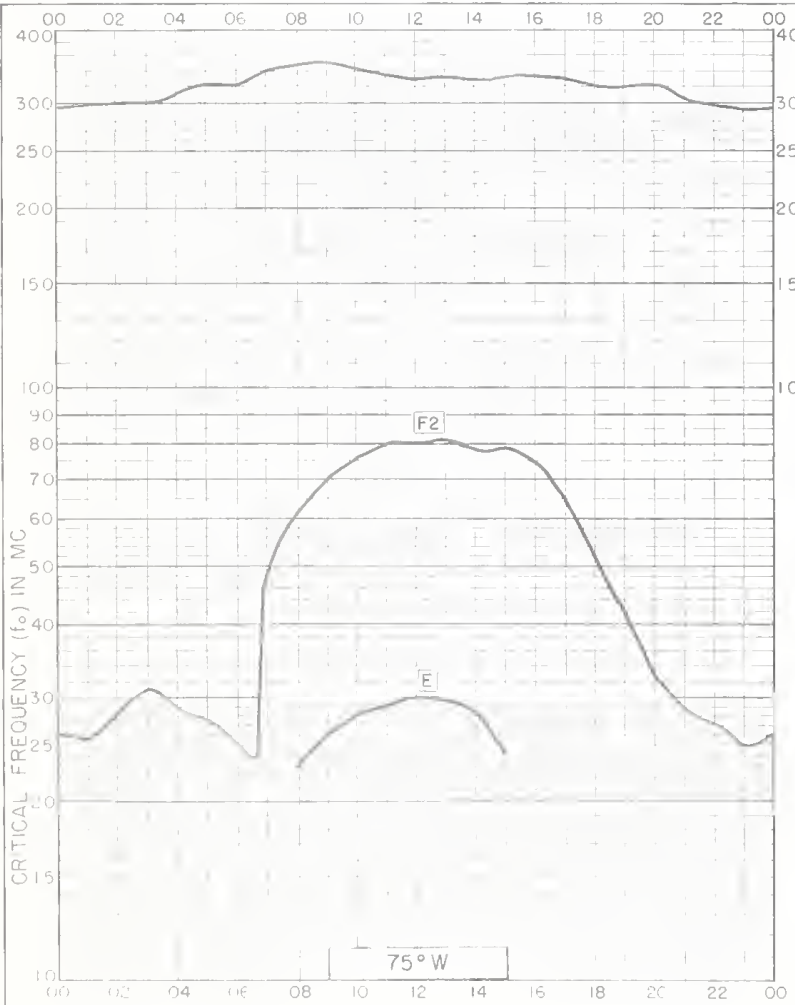

FIg. II. FT. MONMOUTH, NEW JERSEY

$40.4^{\circ} \mathrm{N}, 74.1^{\circ} \mathrm{W} \quad$ NOVEMBER 1961

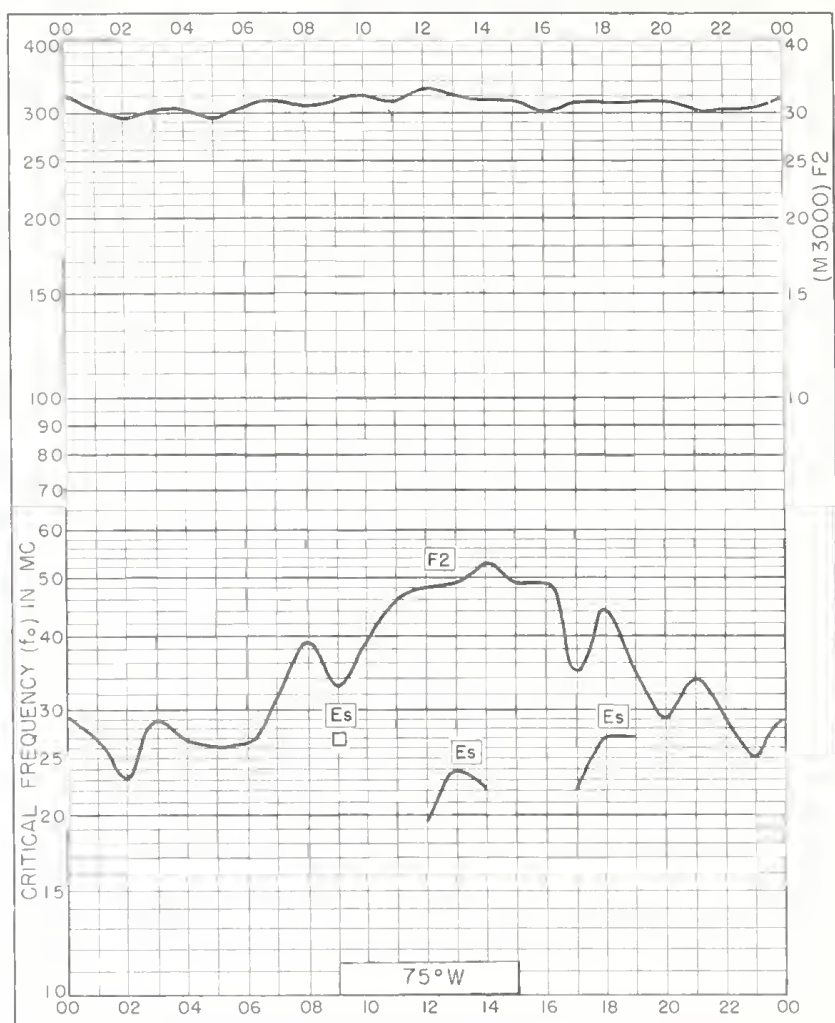

Fig. 10. THULE, GREENLAND

$76.0^{\circ} \mathrm{N}, 68.0^{\circ} \mathrm{W}$ NOVEMBER 1961
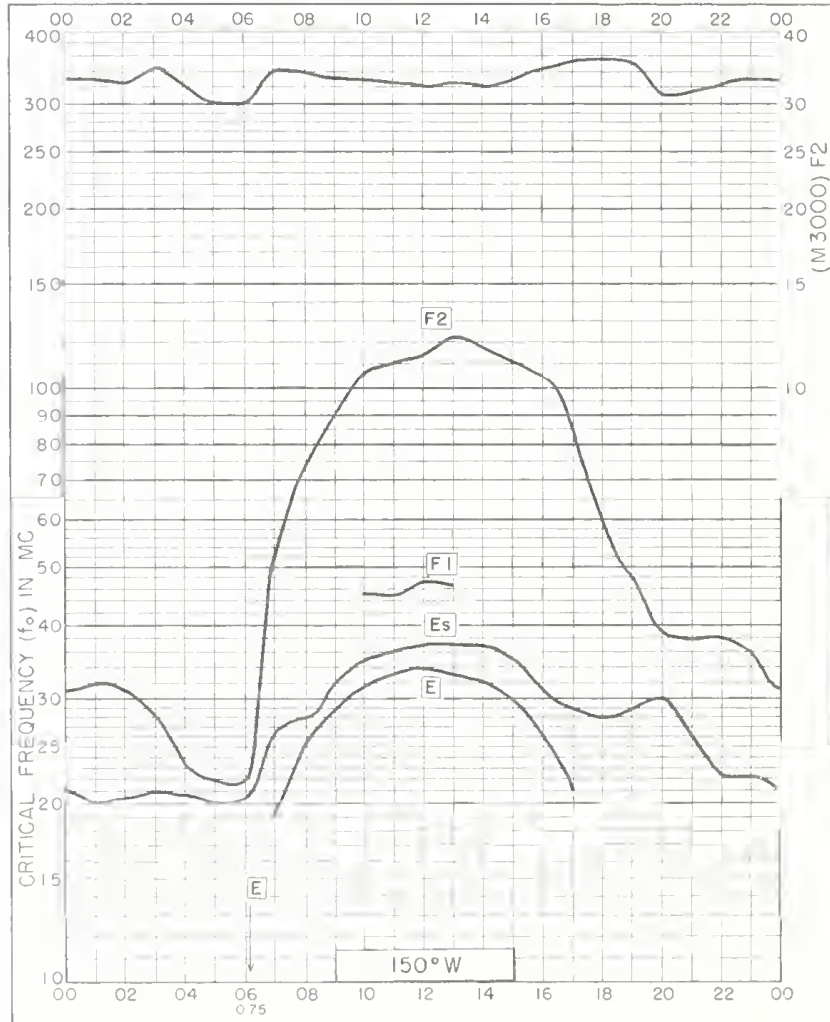

Fig. 12. MAUI, HAWAII

$20.8^{\circ} \mathrm{N}, 156.5^{\circ} \mathrm{W}$ NOVEMBER 1961 


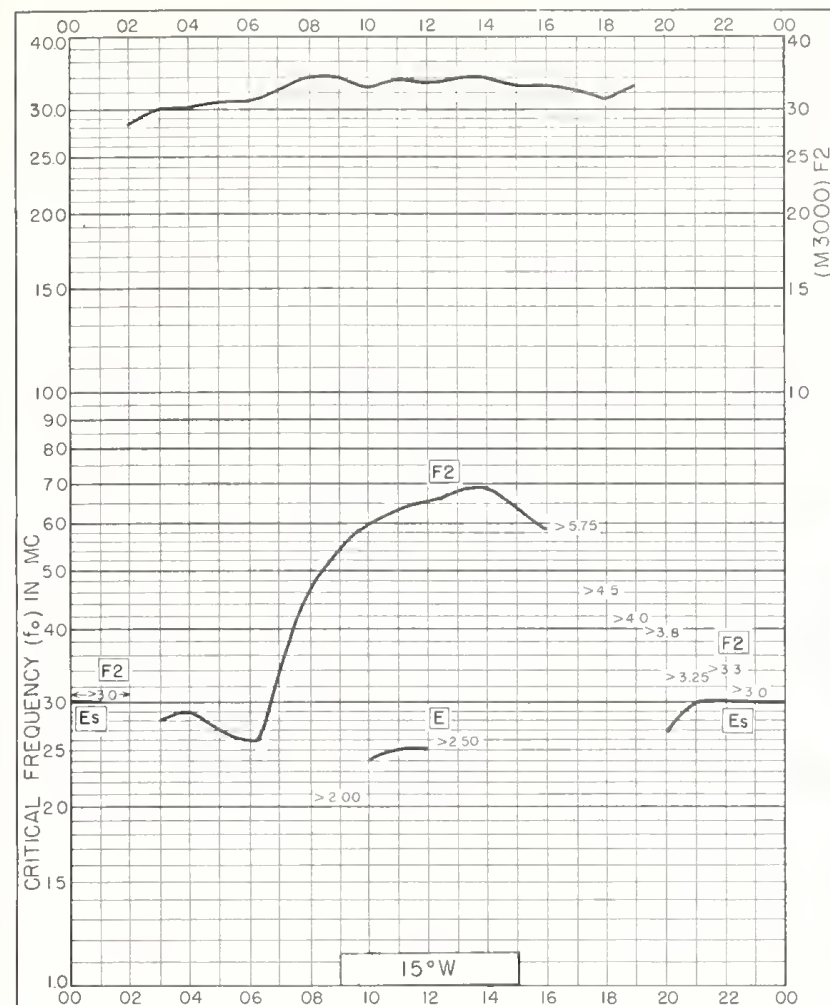

Fig. 13. REYKJAVIK, ICELAND

64. $1^{\circ} \mathrm{N}, 21.8^{\circ} \mathrm{W}$ OCTOBER 1961

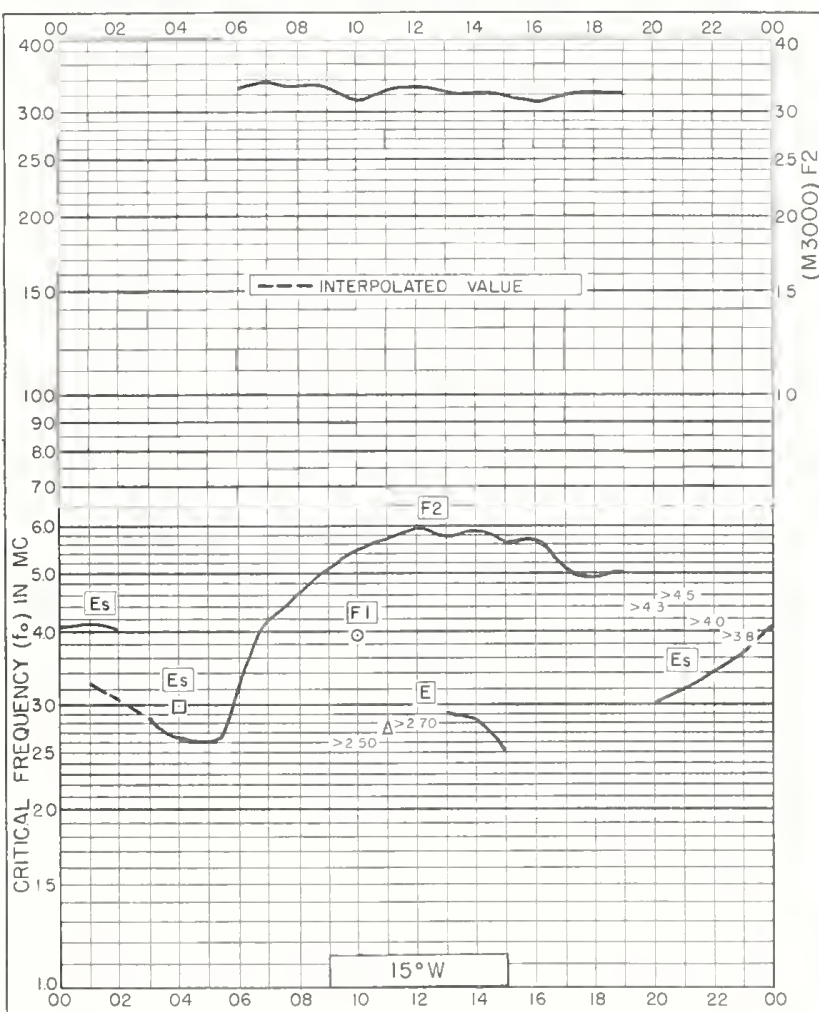

Fig. :5. REYKJAVIK, ICELAND

$64.1^{\circ} \mathrm{N}, 21.8^{\circ} \mathrm{W}$ SEPTEMBER 1961

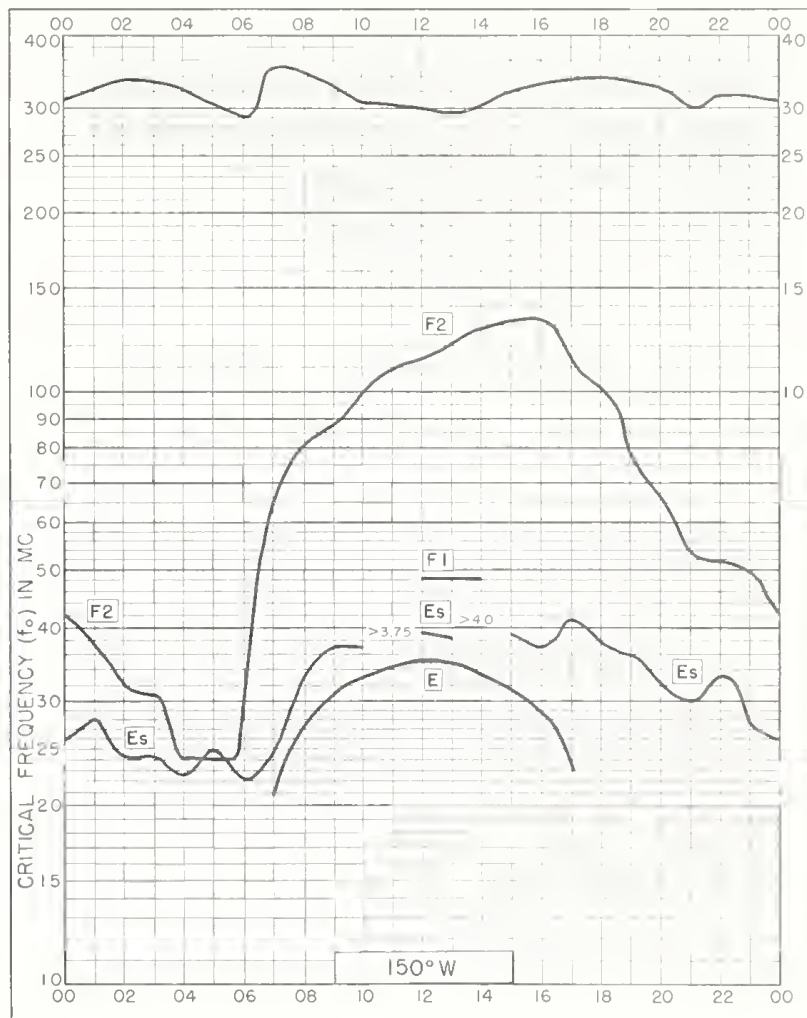

Fig. 14. MAUI, HAWAII

$20.8^{\circ} \mathrm{N}, 156.5^{\circ} \mathrm{W}$

OCTOBER |96|

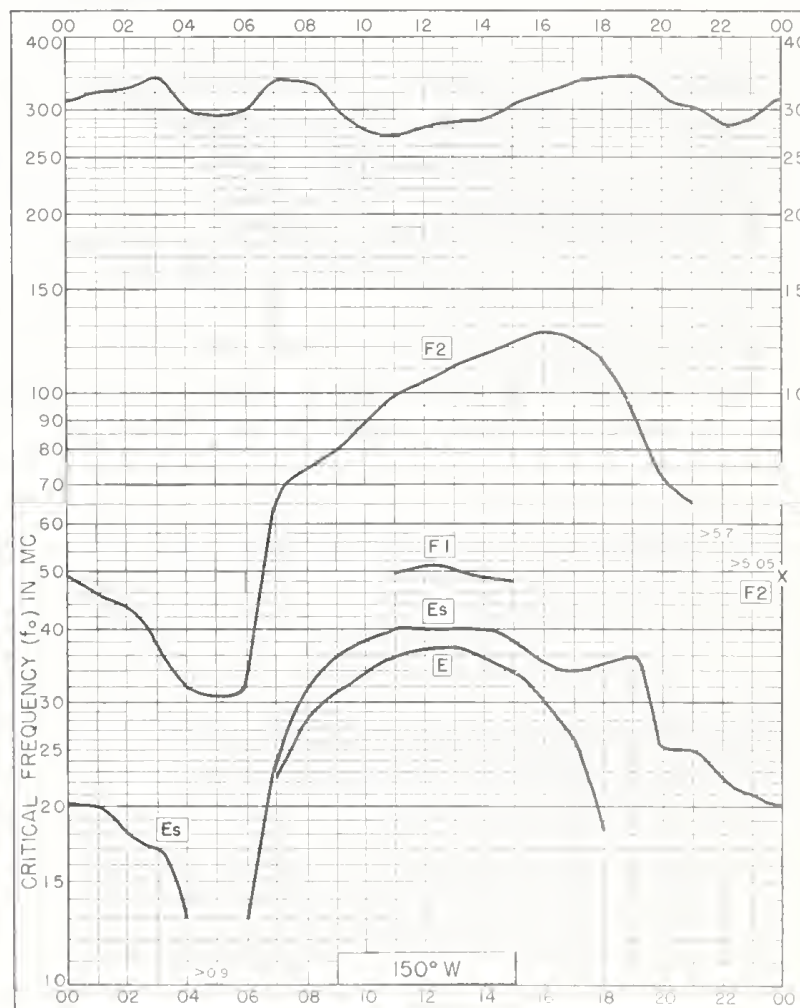

Fig. 16. MAU!, HAWAII

$20.8^{\circ} \mathrm{N}, 156.5^{\circ} \mathrm{W}$ SEPTEMBER 1961 


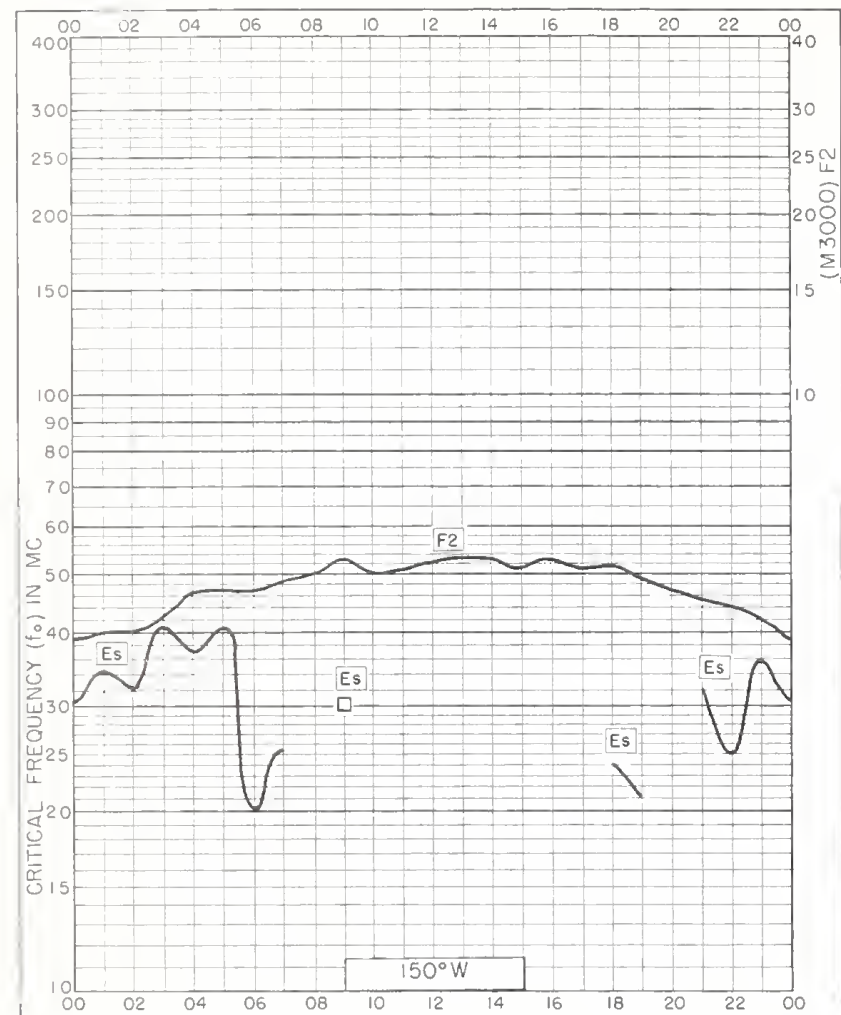

Fig. 17. FAIRBANKS, ALASKA $64.9^{\circ} \mathrm{N}, 147.8^{\circ} \mathrm{W}$

AUGUST 1961

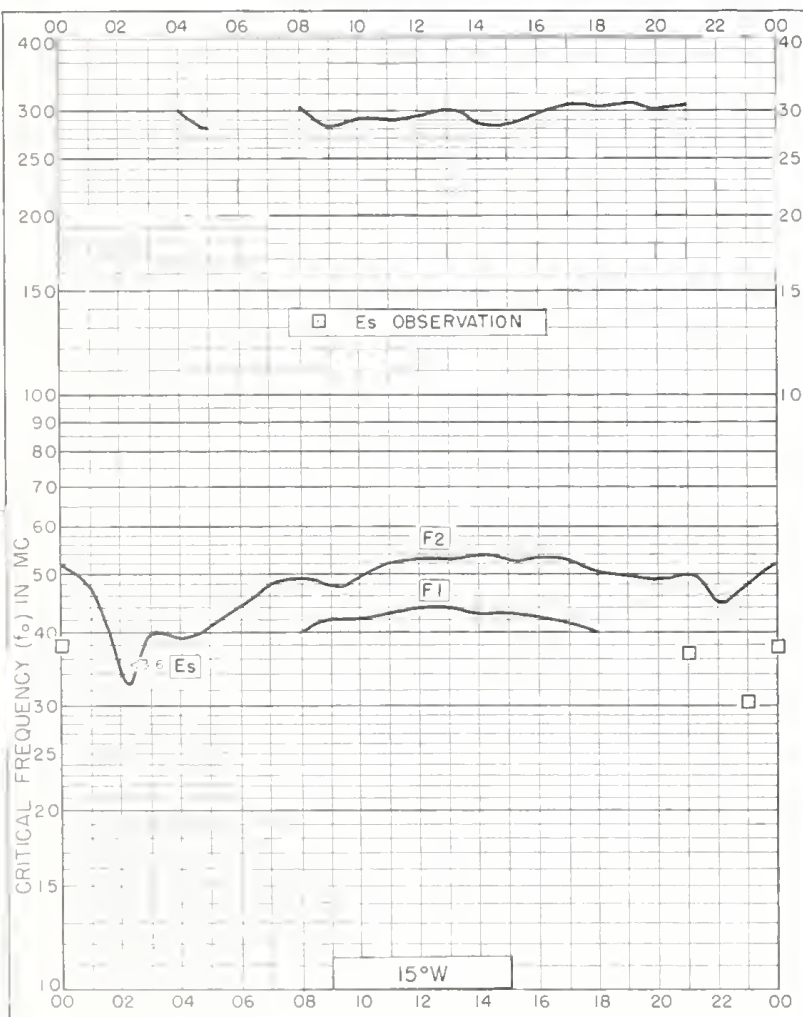

Fig. 19. REYKJAVIK, ICELAND $64.1^{\circ} \mathrm{N}, 21.8^{\circ} \mathrm{W}$

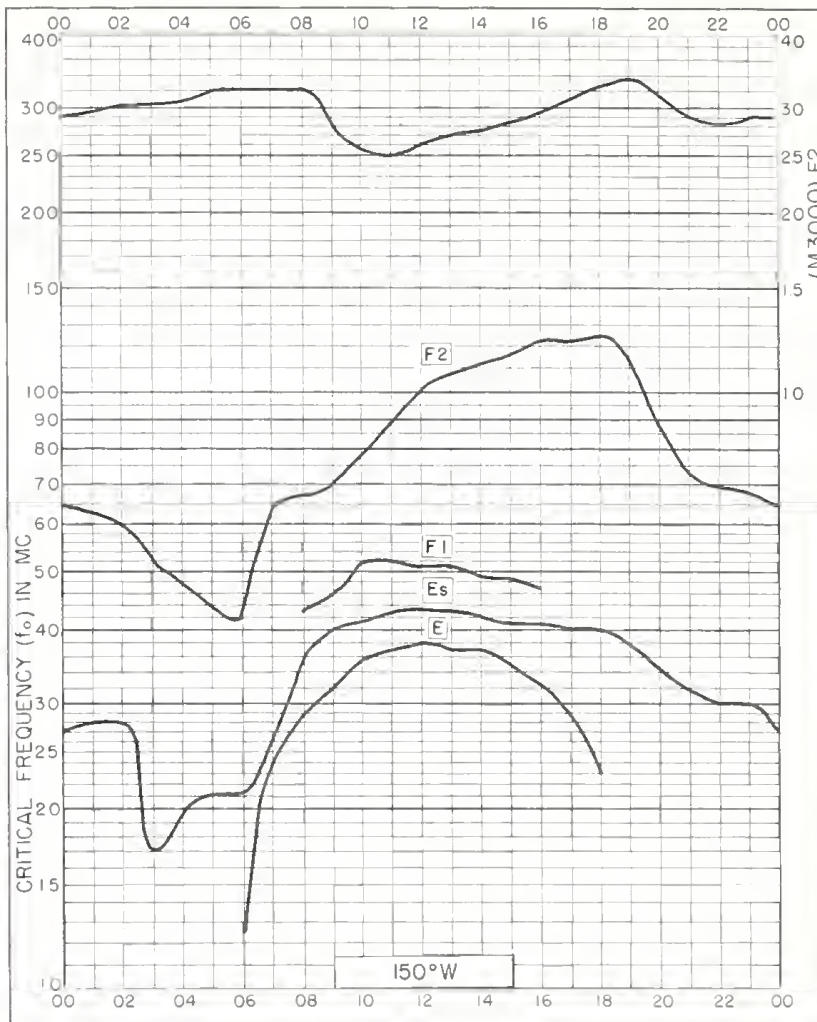

Fig. 18. MAUI, HAWAII

$20.8^{\circ} \mathrm{N}, 156.5^{\circ} \mathrm{W}$

AUGUST 1961

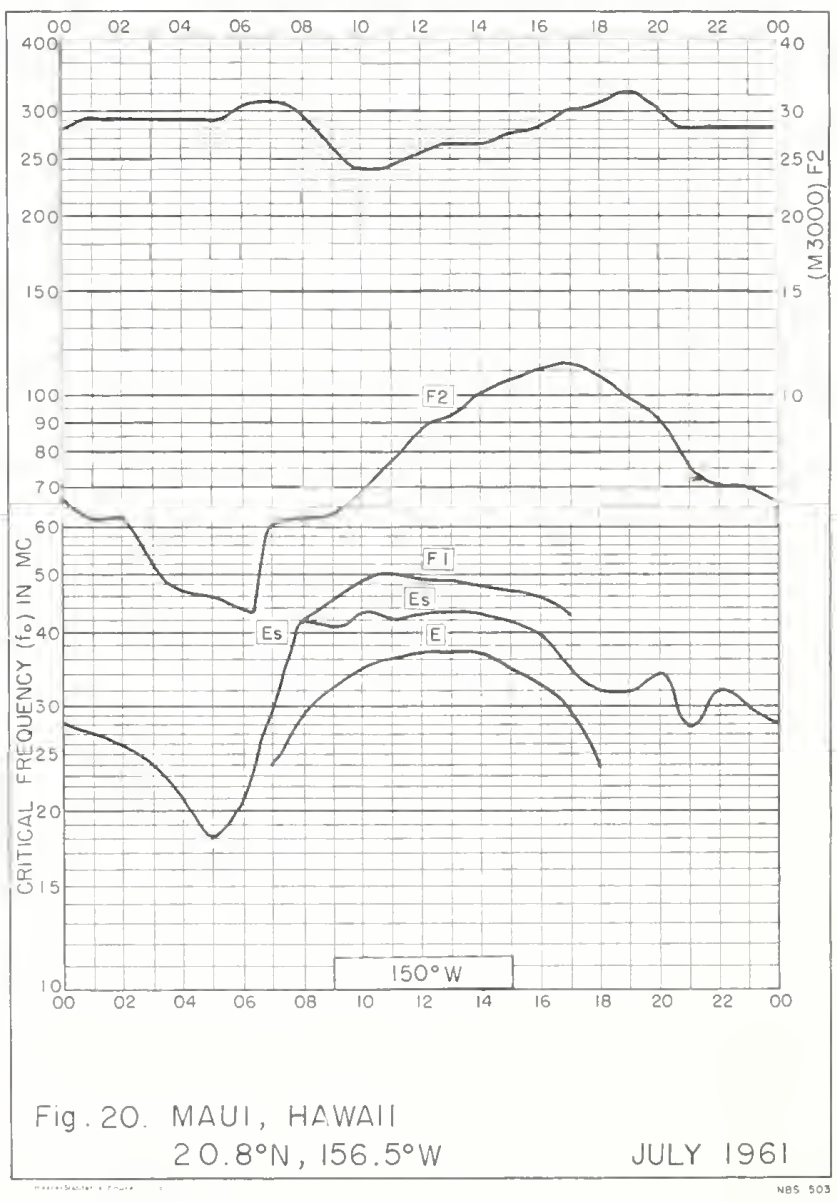




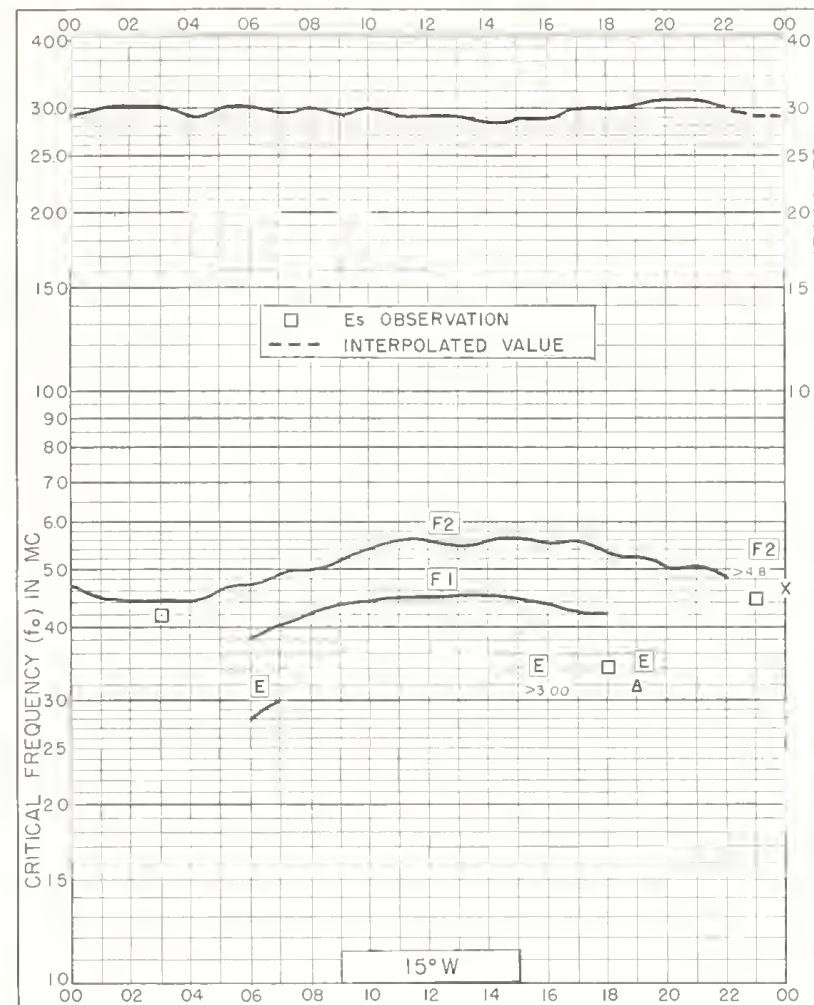

Fig. 21. REYKJAVIK, ICELAND $64.1^{\circ} \mathrm{N}, 21.8^{\circ} \mathrm{W}$ JUNE 1961

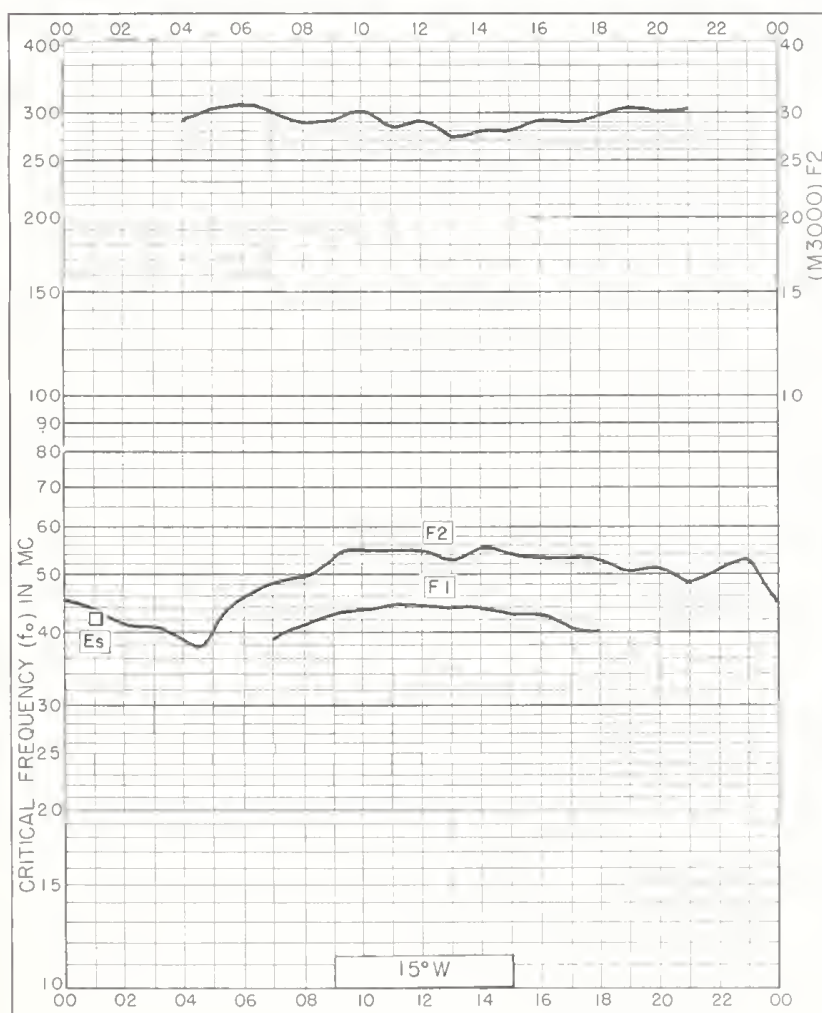

Fig. 23. REYKJAVIK, ICELAND $64.1^{\circ} \mathrm{N}, 21.8^{\circ} \mathrm{W}$

MAY 196I
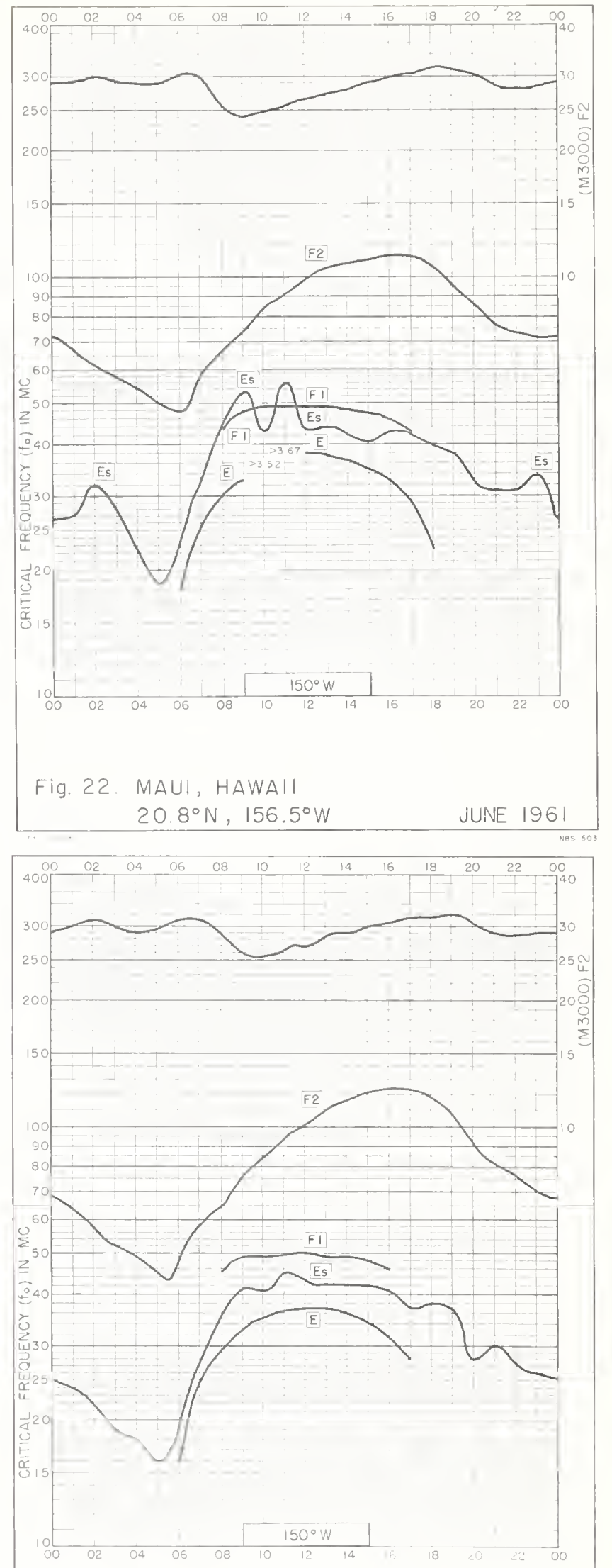

Fig. 24. MAUI, HAWAII $20.8^{\circ} \mathrm{N}, 156.5^{\circ} \mathrm{W}$ MAY 196I 

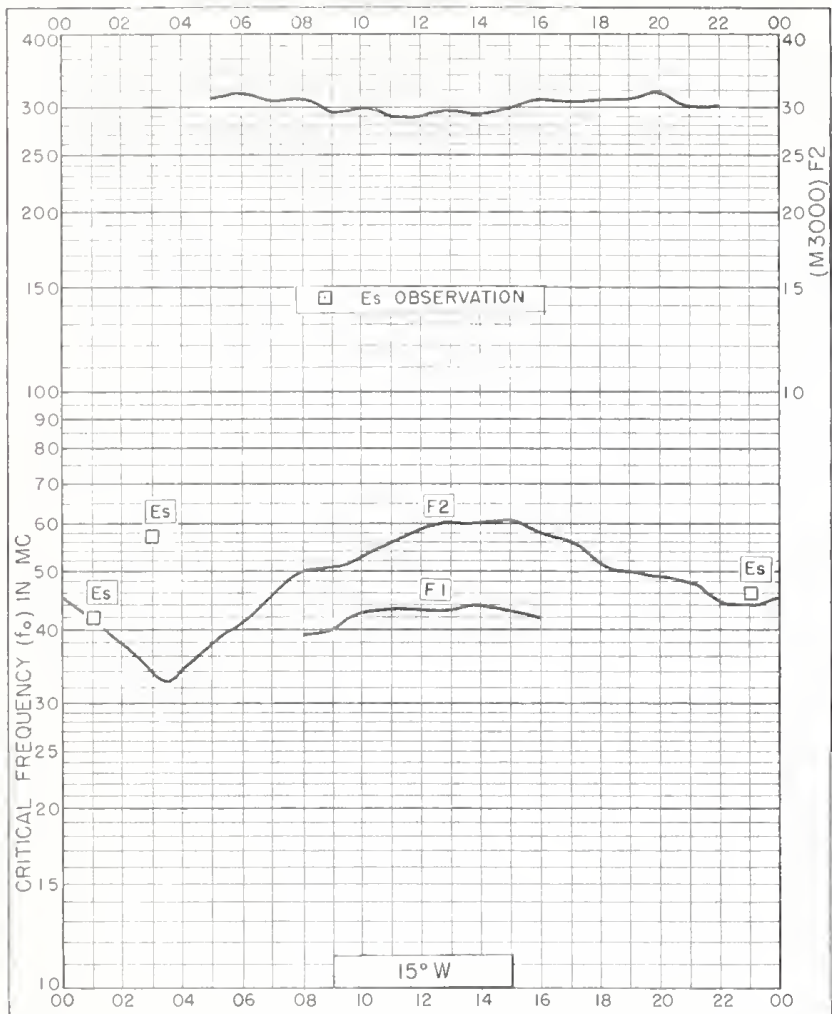

Fig. 25. REYKJAVIK, ICELAND $64.1^{\circ} \mathrm{N}, 21.8^{\circ} \mathrm{W}$ APRIL 1961

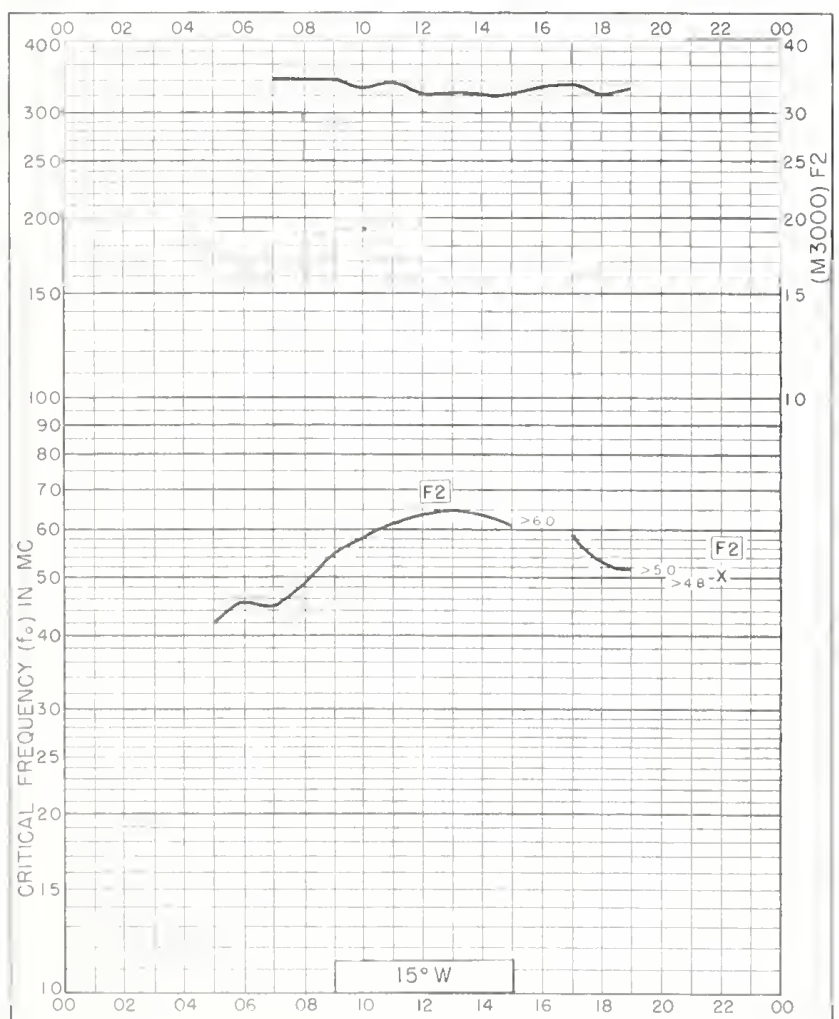

Fig. 26. WHITE SANDS, NEW MEXICO $32.3^{\circ} \mathrm{N}, 106.5^{\circ} \mathrm{W}$ APRIL 196I

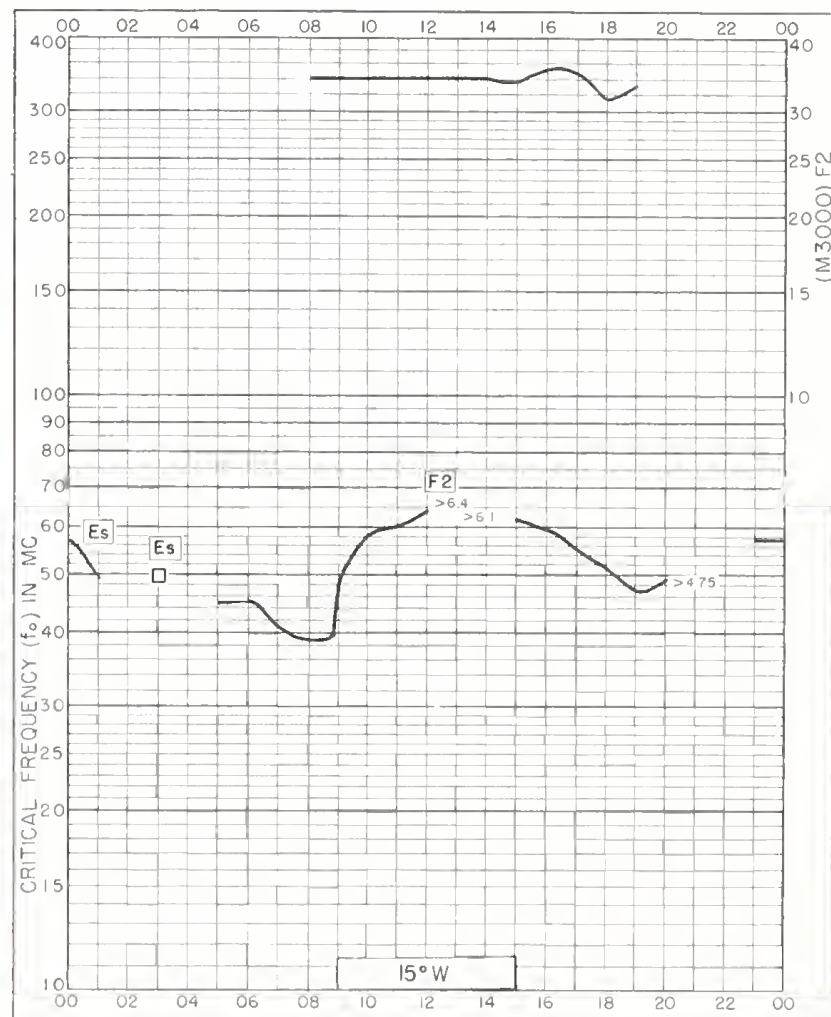

Fig. 28. REYKJAVIK, ICELAND $64.1^{\circ} \mathrm{N}, 21.8^{\circ} \mathrm{W}$ FEBRUARY 1961 


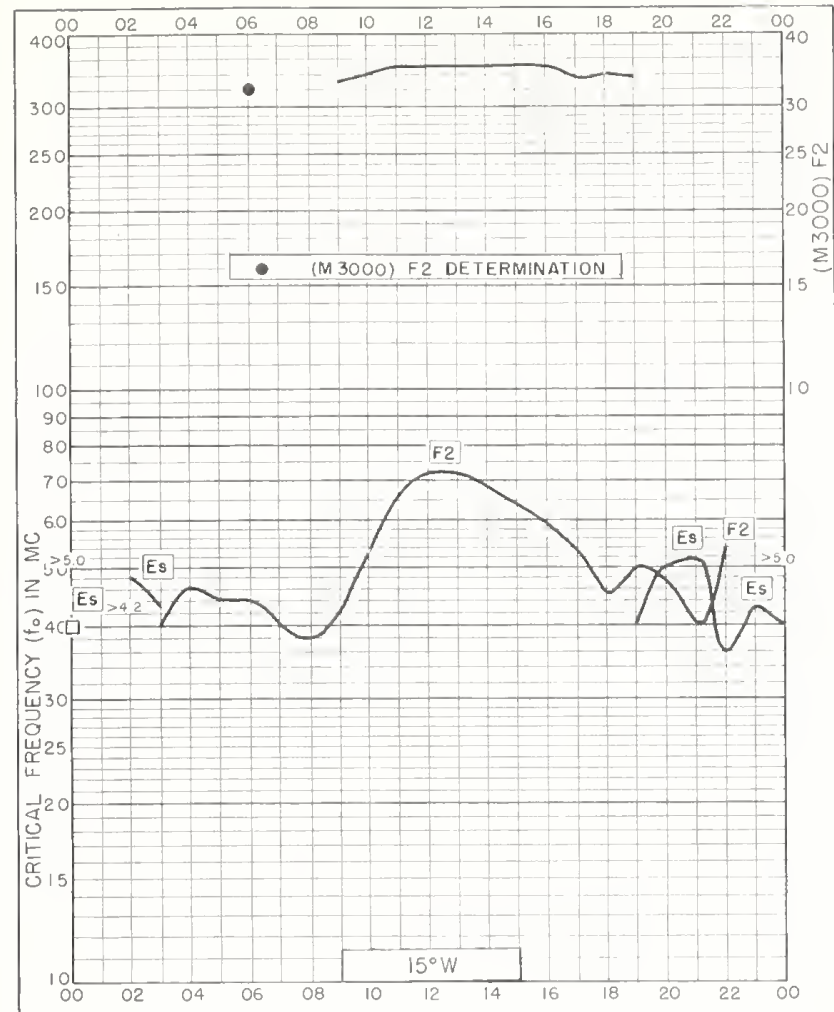

Fig. 29. REYKJAVIK, ICELAND $64.1^{\circ} \mathrm{N}, 21.8^{\circ} \mathrm{W}$ JANUARY 1961

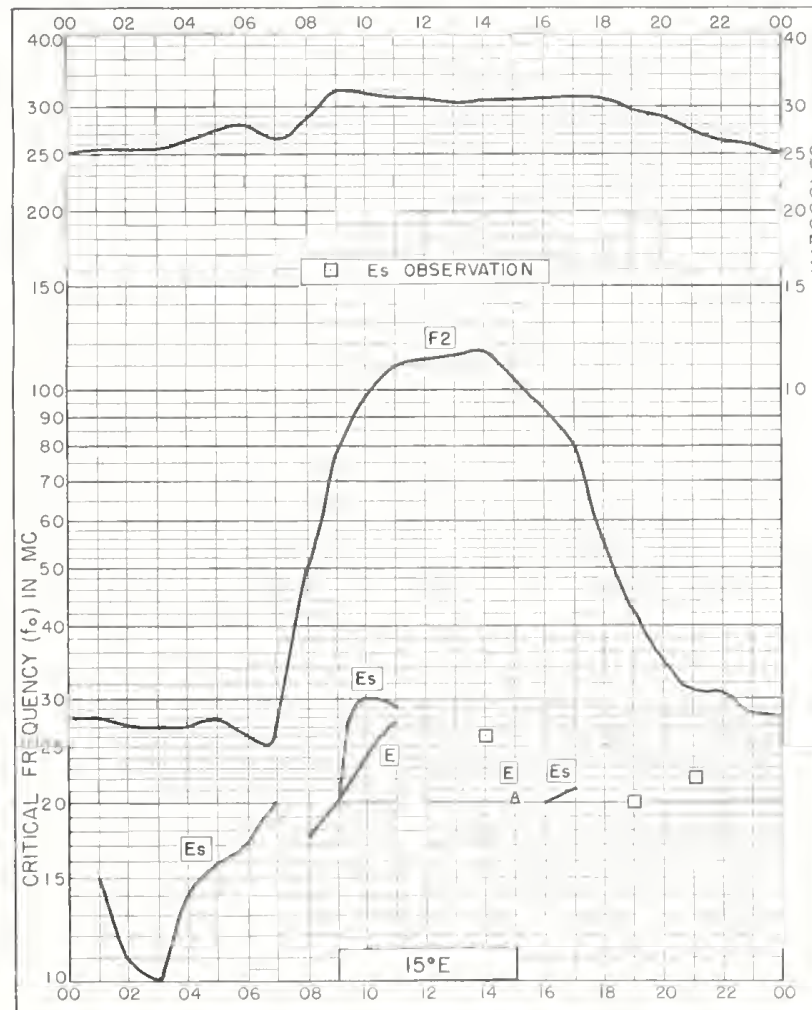

Fig. 31. JULIUSRUH/RUGEN, GERMANY $54.6^{\circ} \mathrm{N}, 13.4^{\circ} \mathrm{E}$

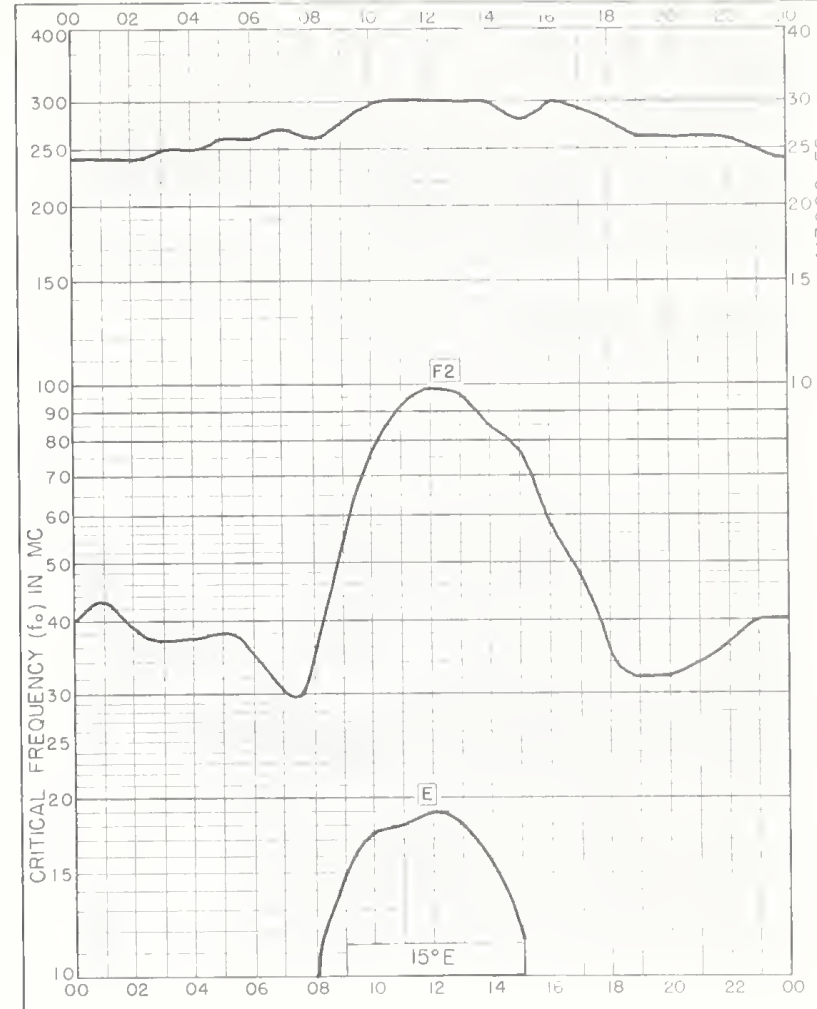

Fig. 30. LYCKSELE, SWEDEN $64.6^{\circ} \mathrm{N}, 18.8^{\circ} \mathrm{E}$

DECEMBER 1959

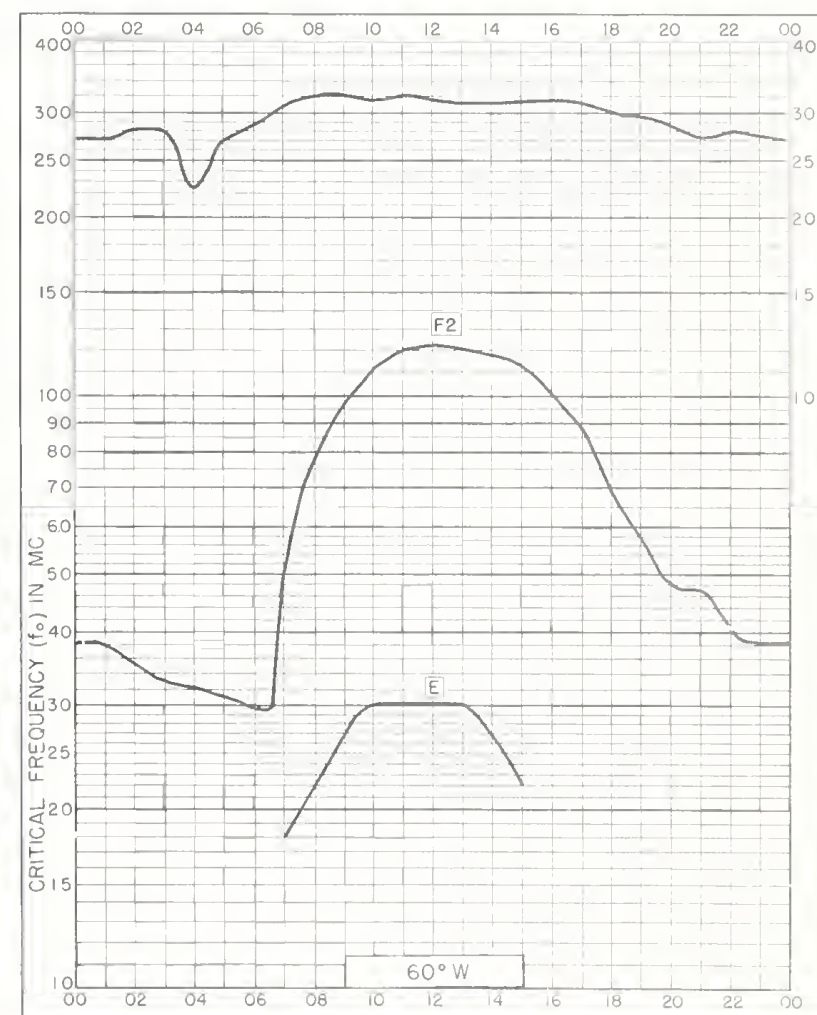

Fig. 32. ST, JOHN'S, NEWFOUNDLAND $47.6^{\circ} \mathrm{N}, 52.7^{\circ} \mathrm{W}$ DECEMBER 1959 


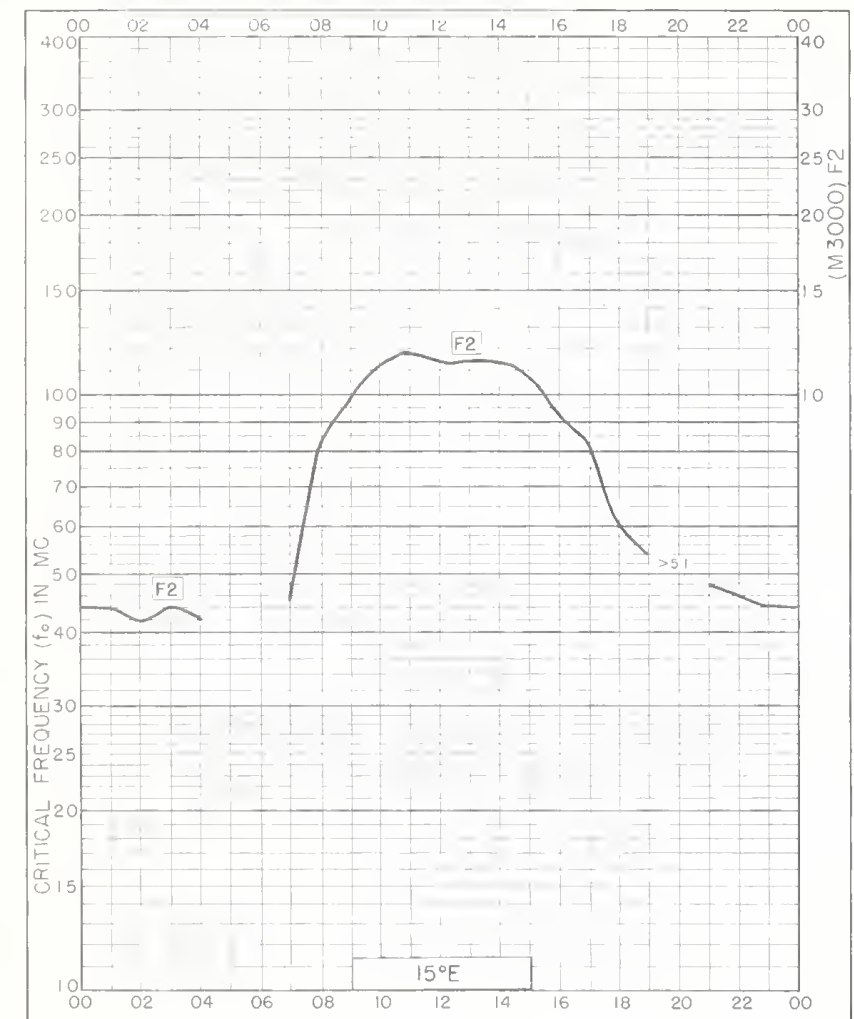

Fig. 33. GRAZ, AUSTRIA $47.1^{\circ} \mathrm{N}, 15.5^{\circ} \mathrm{E}$

DECEMBER 1959

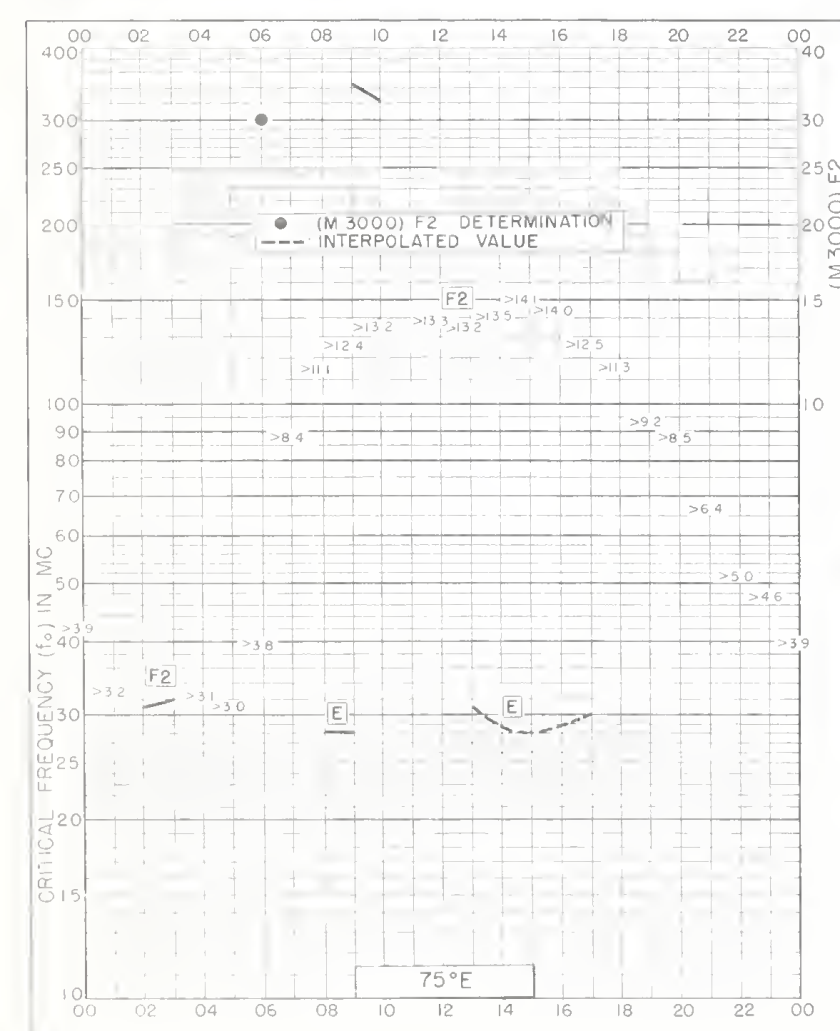

Fig 35. DELHI, INDIA

$28.6^{\circ} \mathrm{N}, 77.2^{\circ} \mathrm{E}$

DECEMBER 1959

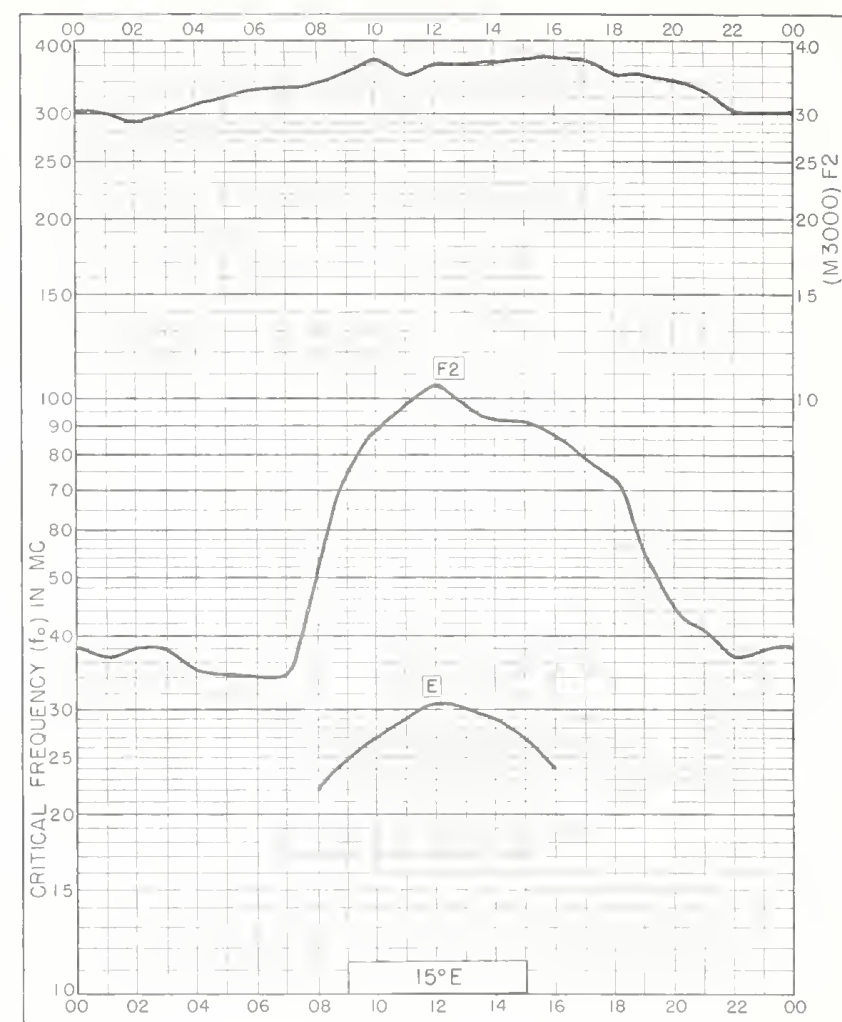

Fig. 34. SOTTENS, SWITZERLAND

$46.6^{\circ} \mathrm{N}, 6.7^{\circ} \mathrm{E} \quad$ DECEMBER 1959

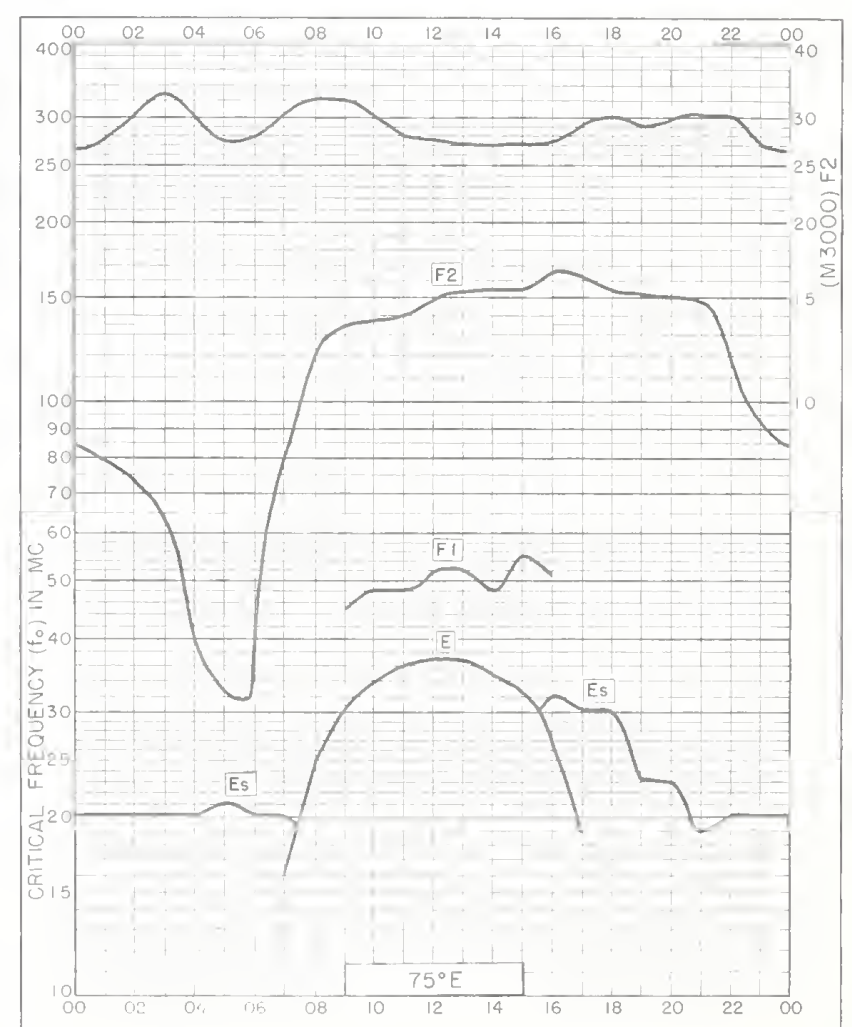

FIg. 36. AHMEDABAD, INDIA

23. $0^{\circ} \mathrm{N}, 72.6^{\circ} \mathrm{E} \quad$ DECEMBER 1959 


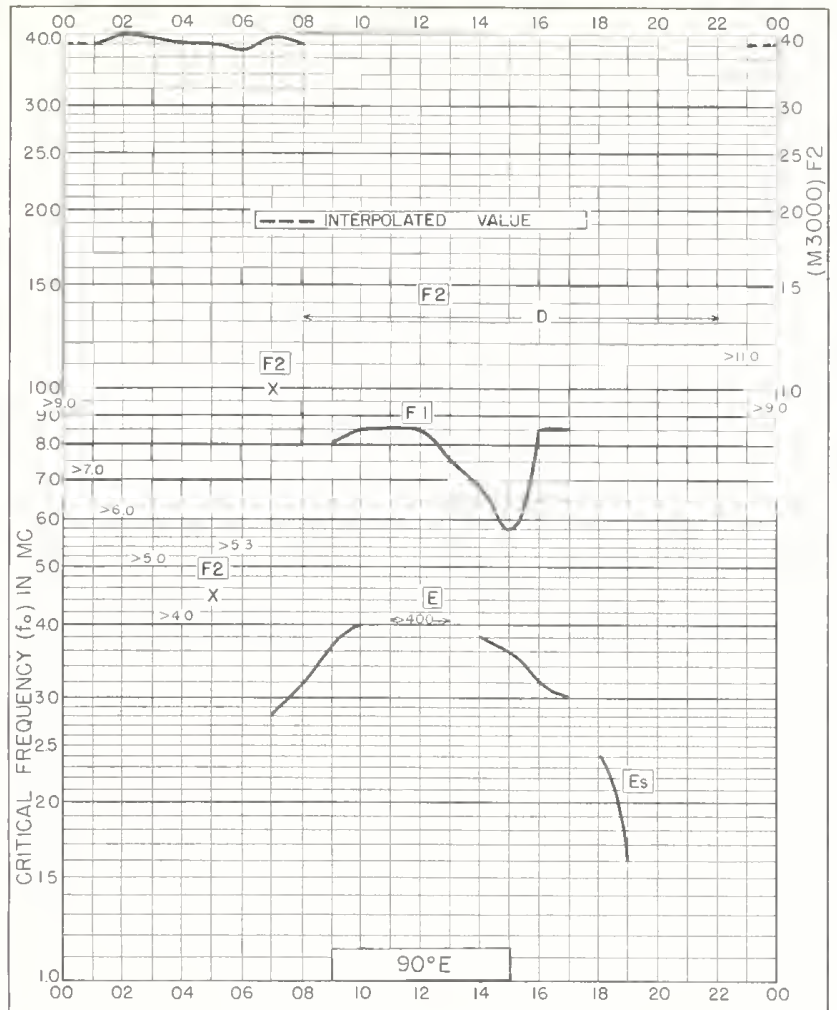

Fig. 37. CALCUTTA, INDIA $23.0^{\circ} \mathrm{N}, 88.6^{\circ} \mathrm{E} \quad$ DECEMBER 1959

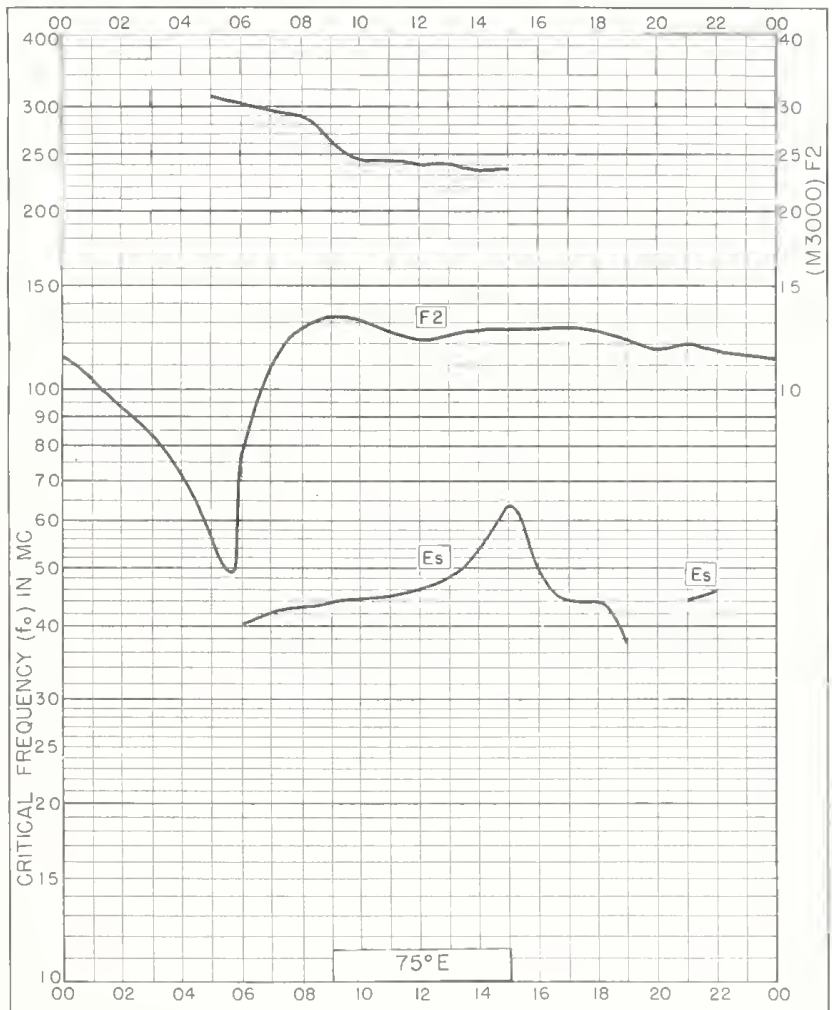

Fig. 39. MADRAS, INDIA

$13.1^{\circ} \mathrm{N}, 80.3^{\circ} \mathrm{E} \quad$ DECEMBER 1959
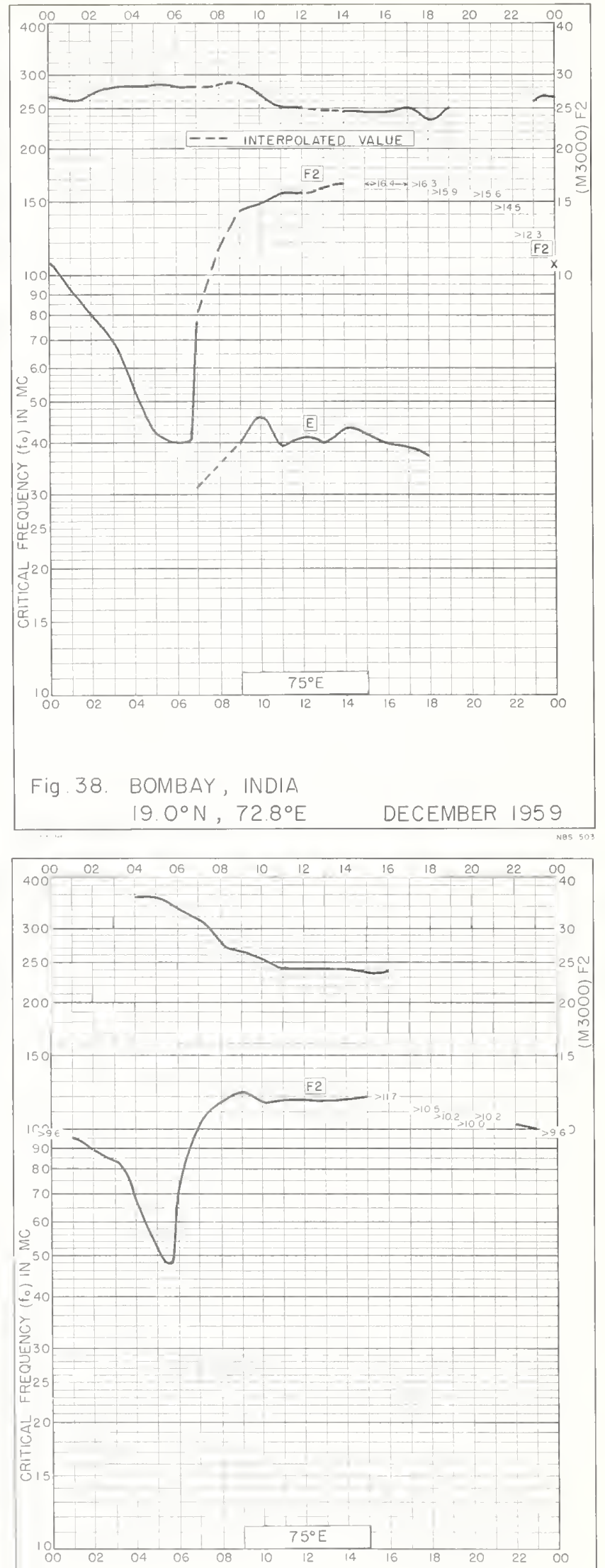

Fig. 40. TIRUCHY, INDIA $10.8^{\circ} \mathrm{N}, 78.7^{\circ} \mathrm{E}$ 


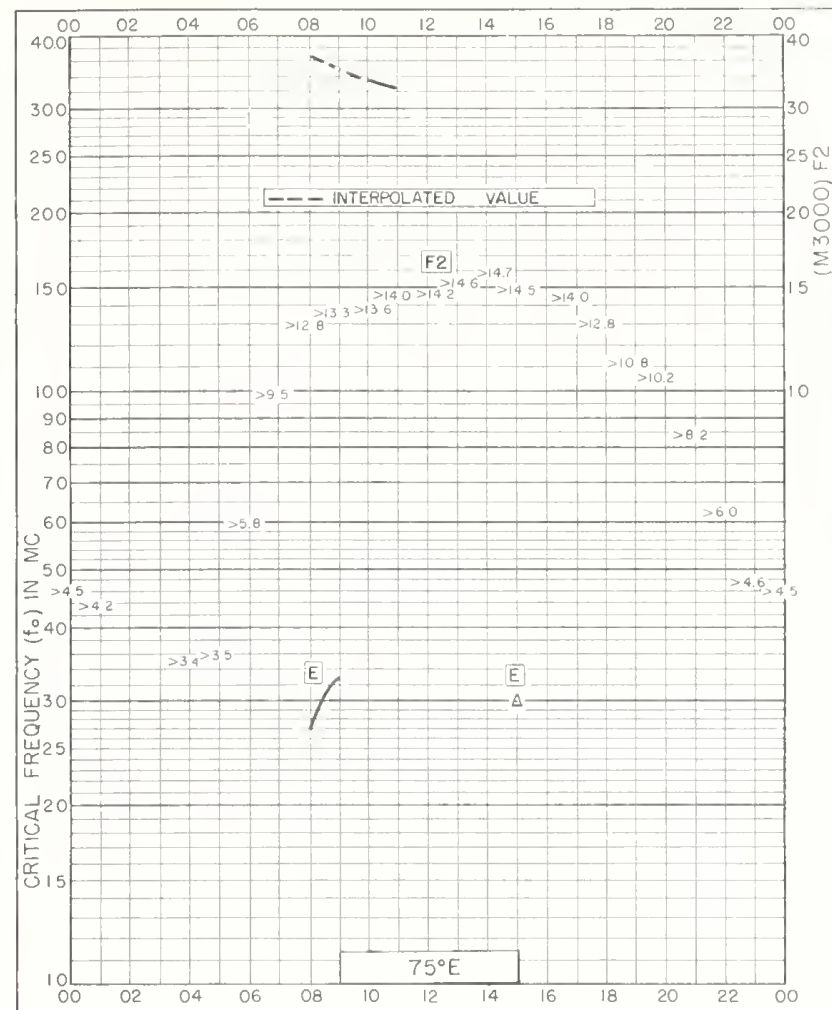

Fig. 45. DELHI, INDIA

$$
28.6^{\circ} \mathrm{N}, 77.2^{\circ} \mathrm{E}
$$

NOVEMBER 1959

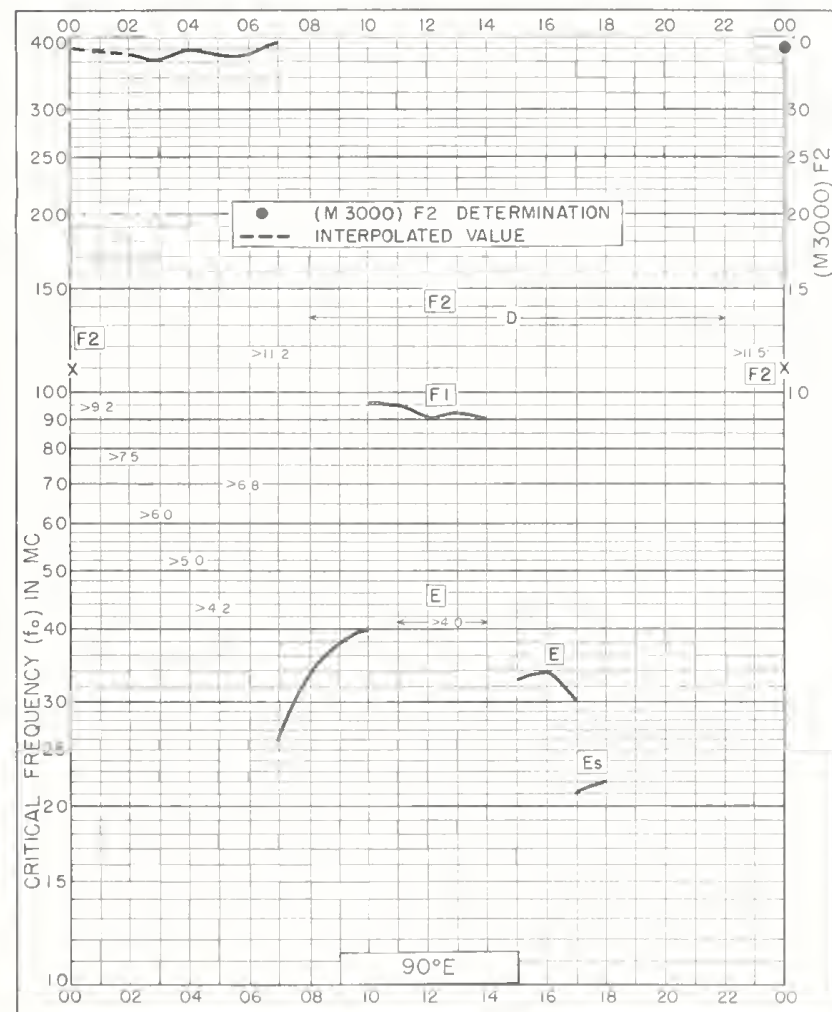

Fig. 47. CALCUTTA, INLIA $23.0^{\circ} \mathrm{N}, 88.6^{\circ} \mathrm{E}$ NOVEMBER 1959

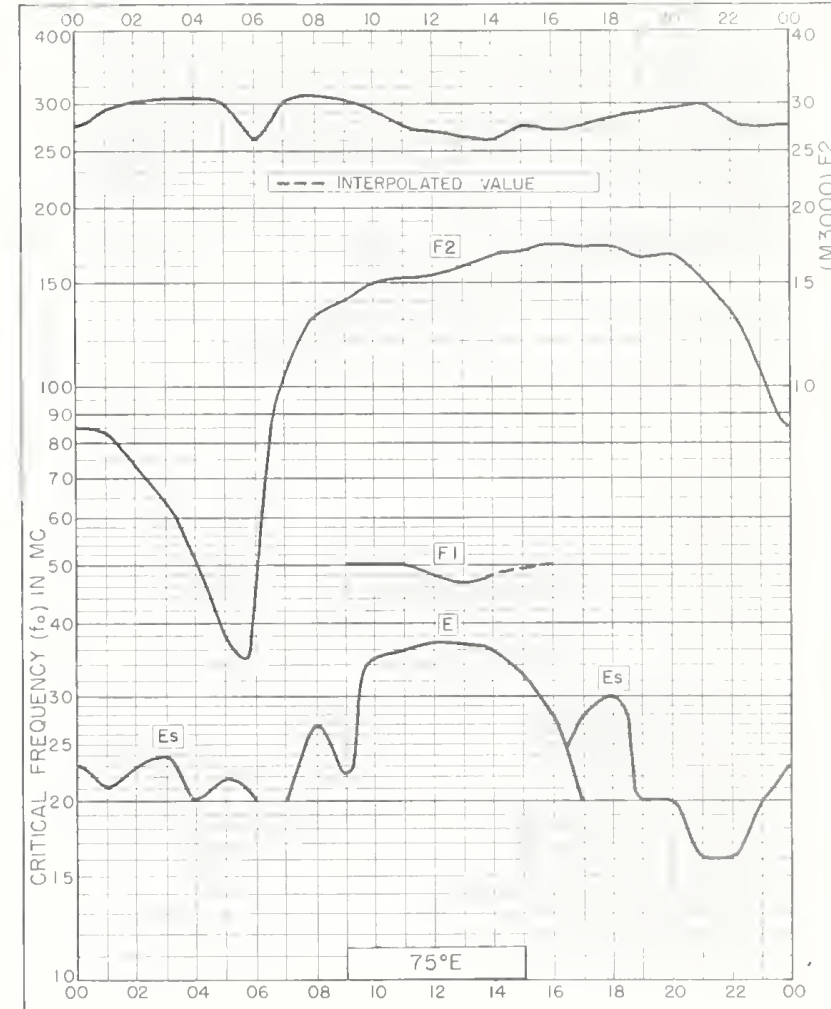

Fig. 46. AHMEDABAD, INDIA $23.0^{\circ} \mathrm{N}, 72.6^{\circ} \mathrm{E} \quad$ NOVEMBER 1959

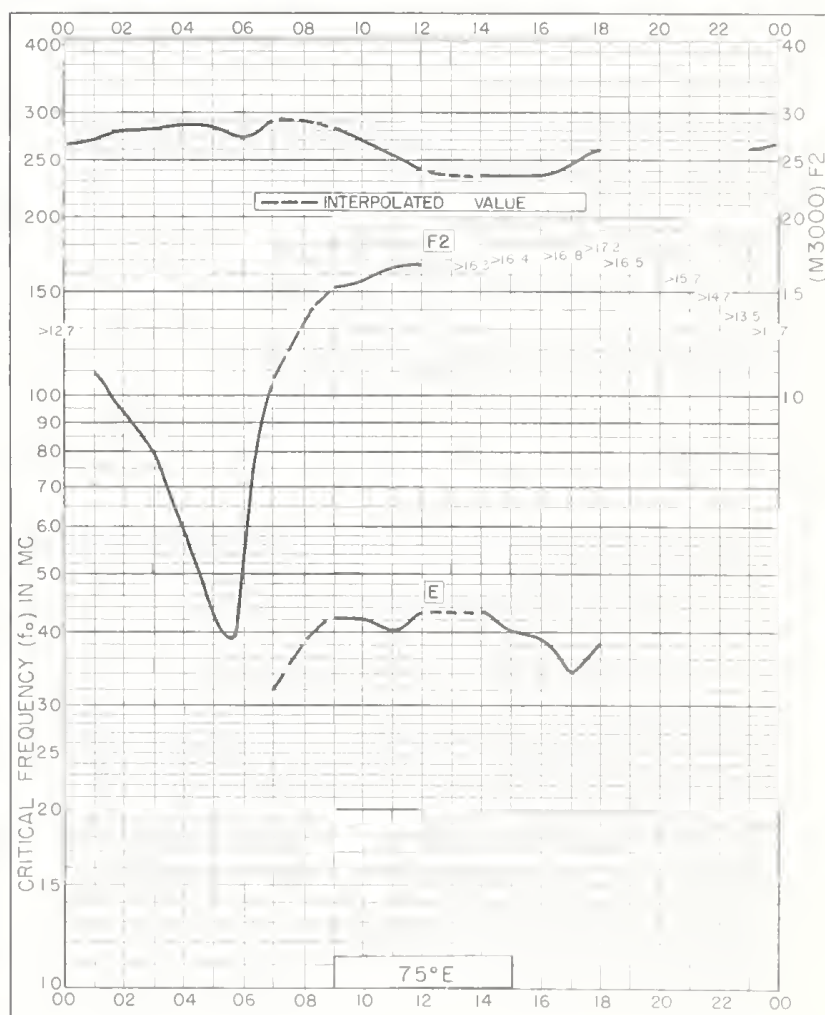

Fig. 48. BOMBAY, INDIA $19.0^{\circ} \mathrm{N}, 72.8^{\circ} \mathrm{E}$ NOVEMBFR 1959 


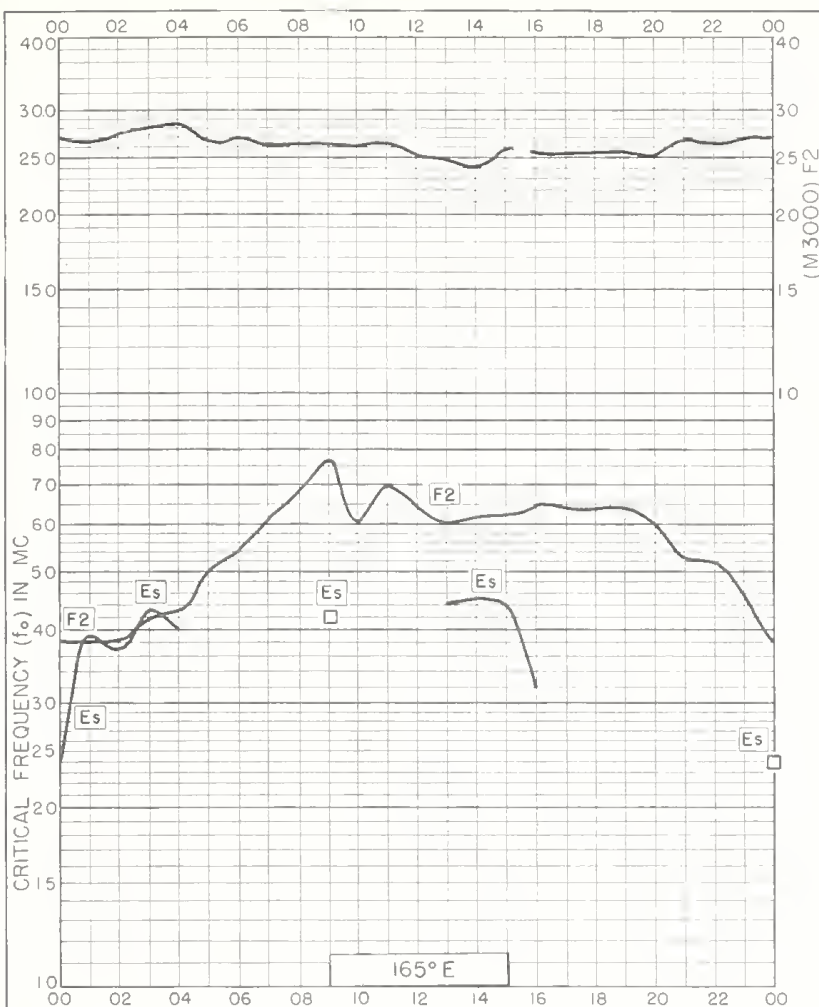

Fig. 53. CAPE HALLETT

$72.3^{\circ} \mathrm{S}, 170.2^{\circ} \mathrm{E} \quad$ NOVEMBER 1959

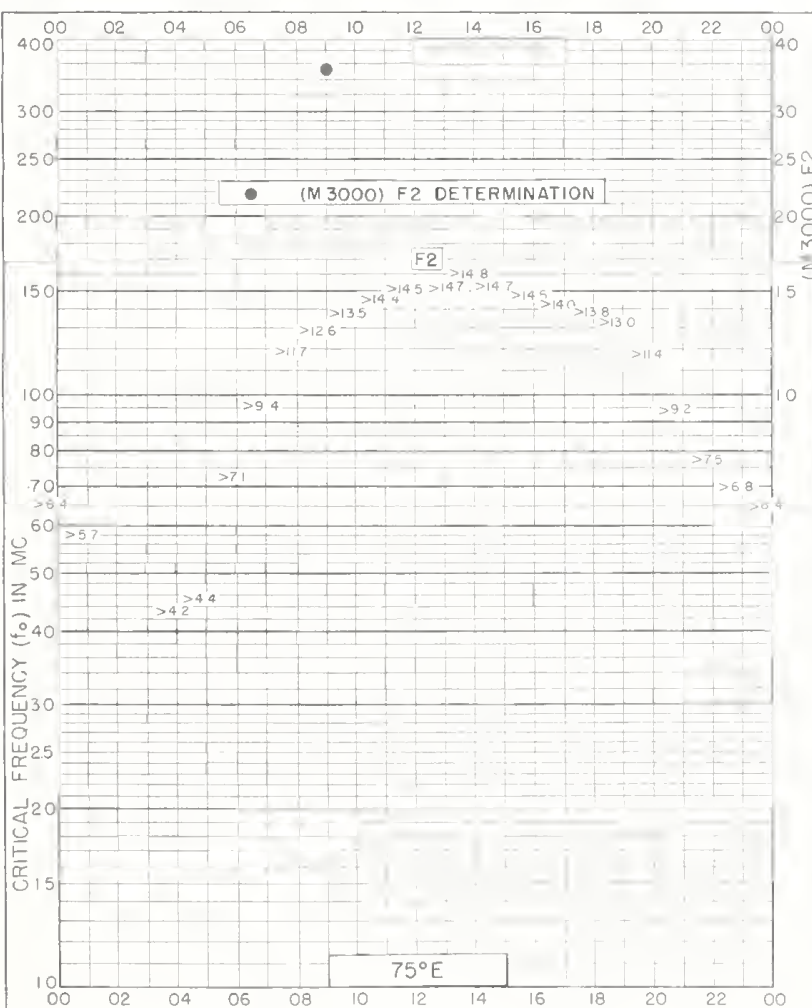

Fig. 55. DELHI, INCIA

28. $6^{\circ} \mathrm{N}, 77.2^{\circ} \mathrm{E} \quad$ OCTOBER 1959

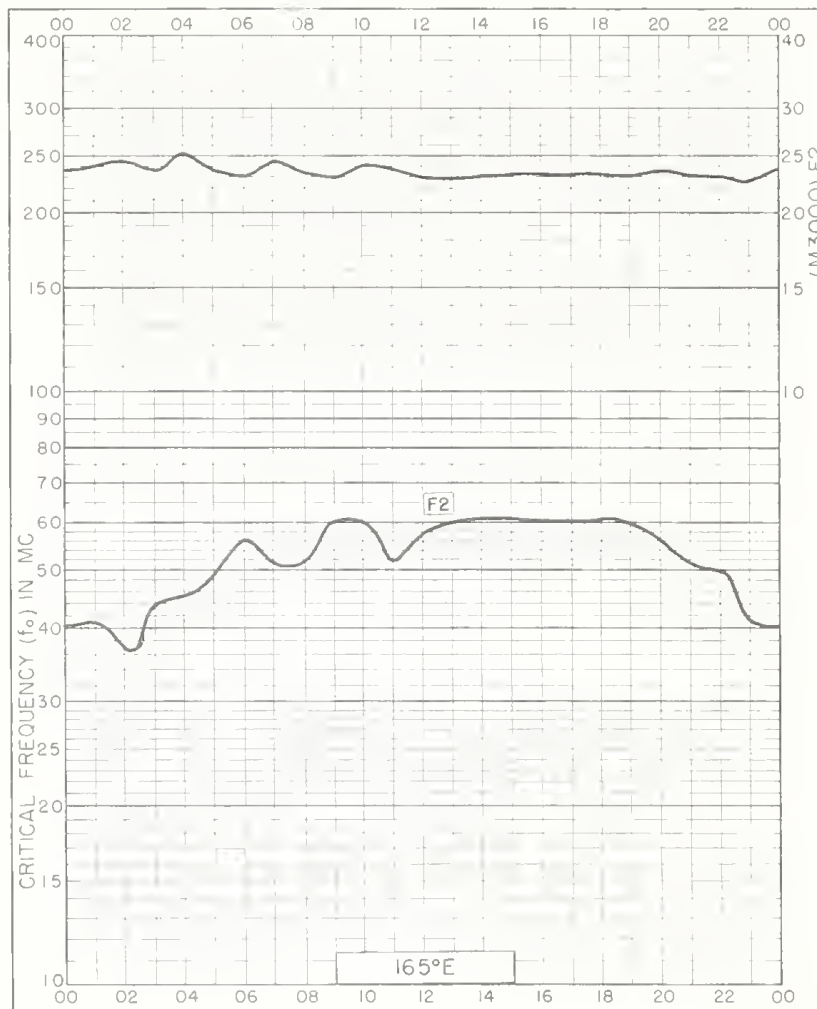

Fig. 54. SCOTT BASE

$77.9^{\circ} \mathrm{S}, 166.8^{\circ} \mathrm{E} \quad$ NOVEMBER 1959

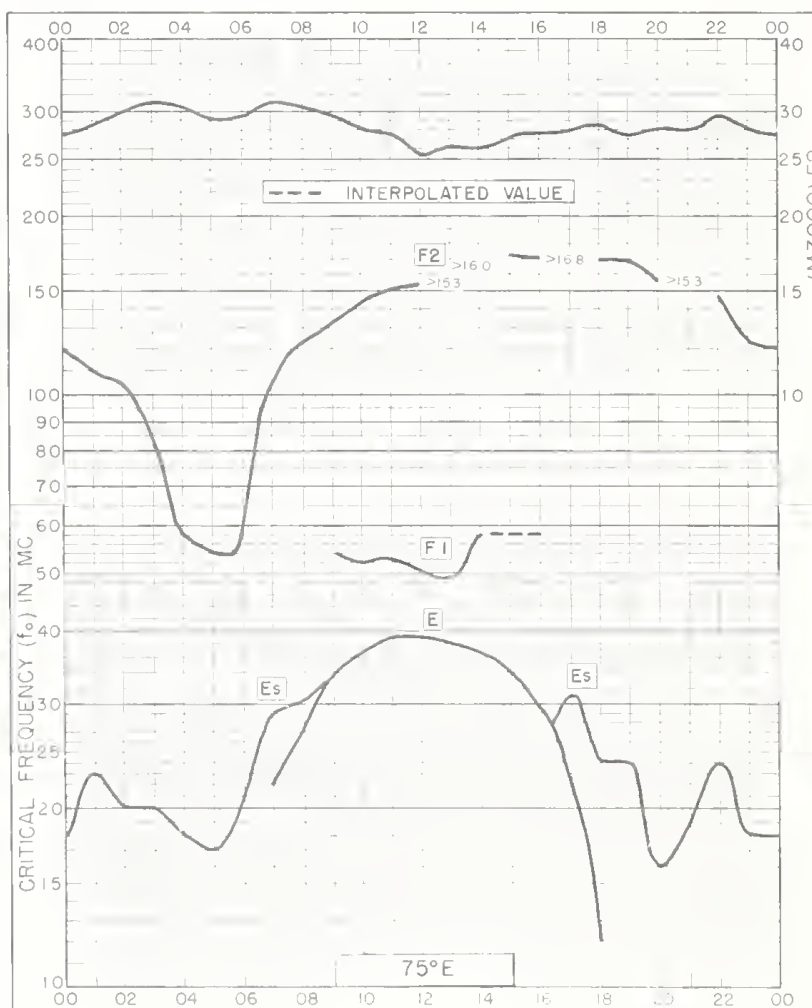

Fig 56. AHMEDABAD, INDIA 23. $0^{\circ} \mathrm{N}, 72.6^{\circ} \mathrm{E}$ OCTOBER 1959 


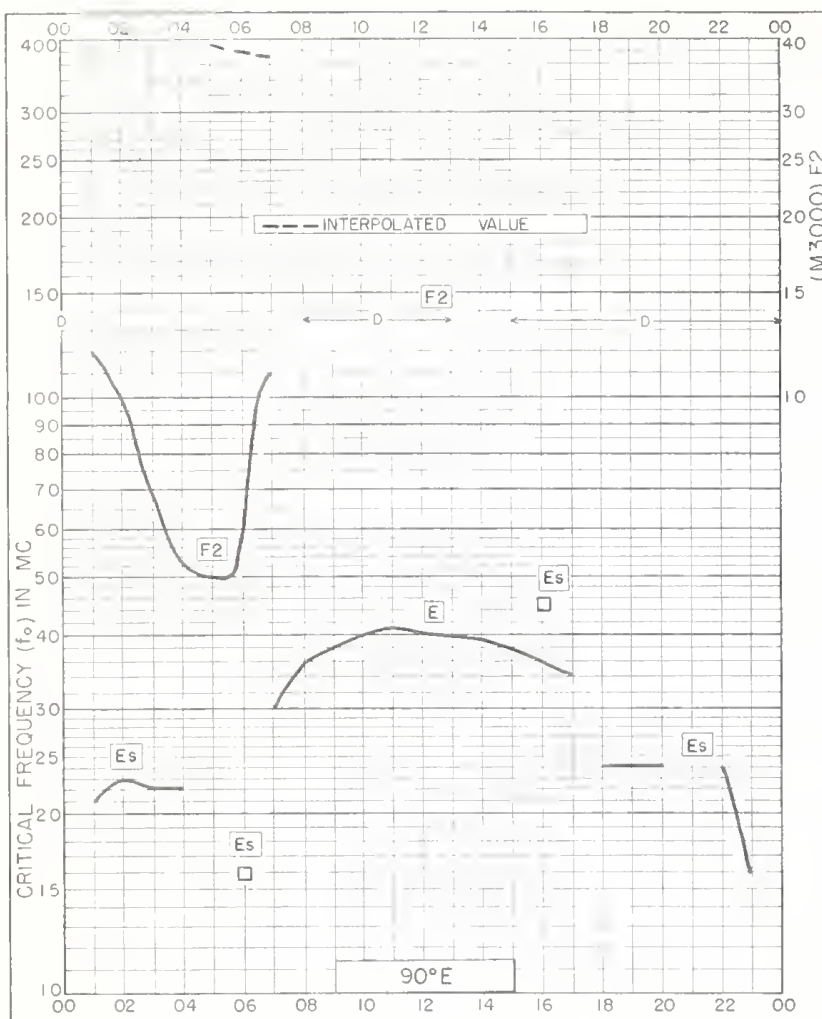

Fig. 57. CALCUTTA, INDIA $23.0^{\circ} \mathrm{N}, 88.6^{\circ} \mathrm{E}$ OCTOBER 1959

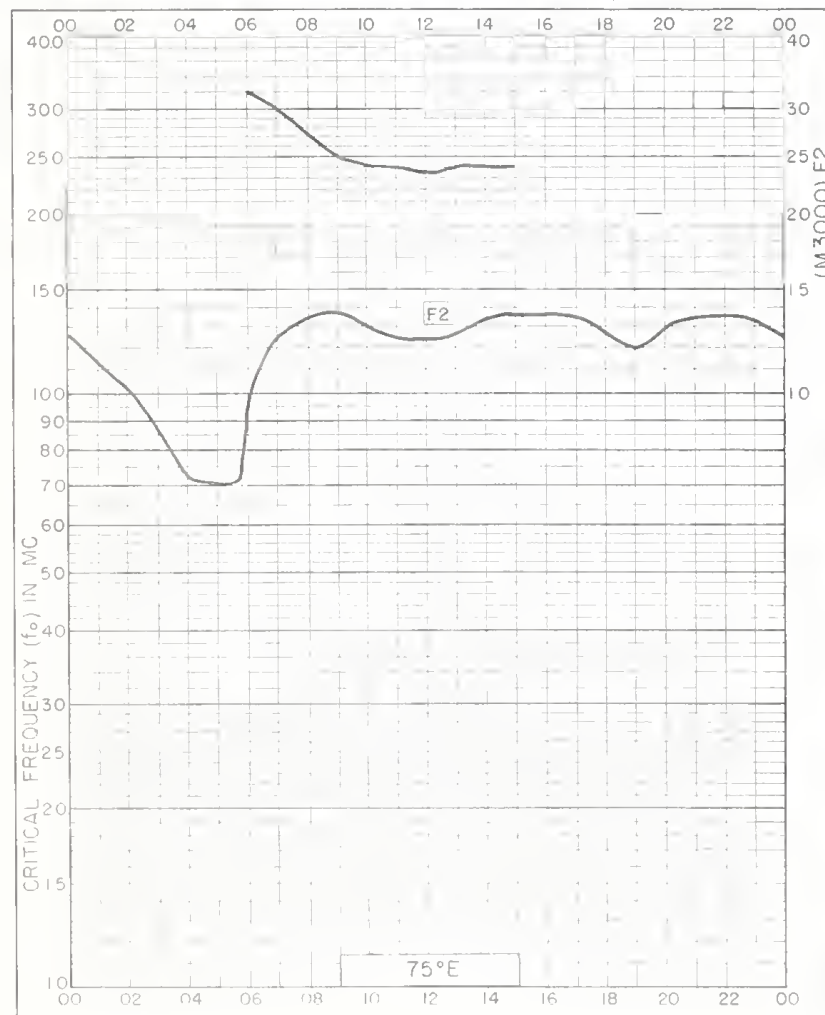

Fig 59. MADRAS, INDIA $13.1^{\circ} \mathrm{N}, 80.3^{\circ} \mathrm{E}$

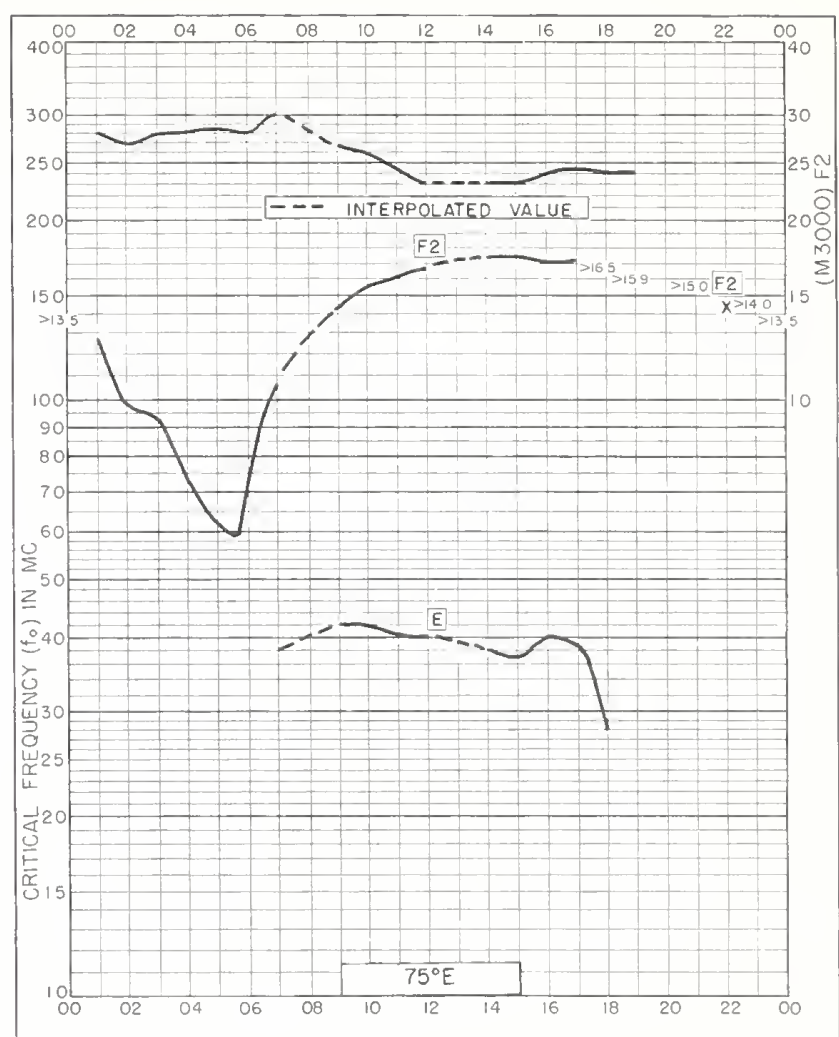

Fig. 58. BOMBAY, INDIA $19.0^{\circ} \mathrm{N}, 72.8^{\circ} \mathrm{E}$ OCTOBER 1959

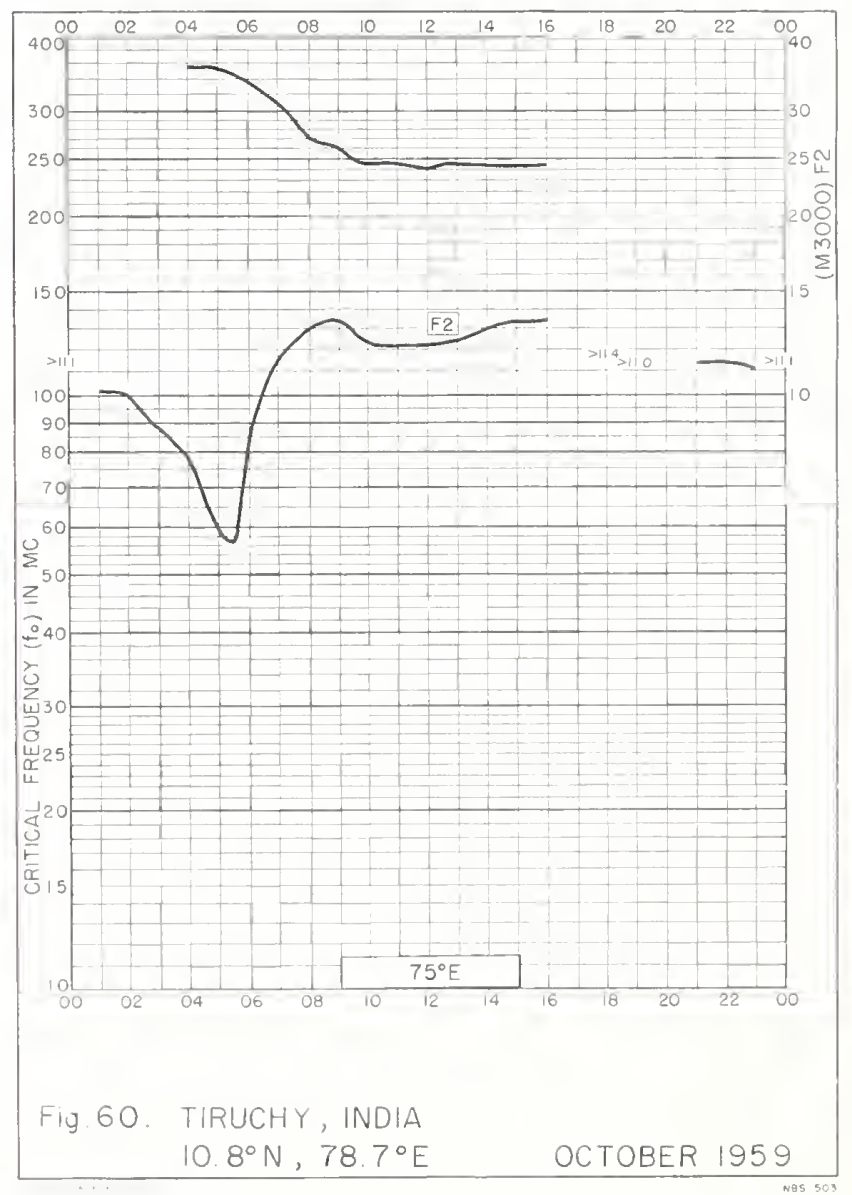




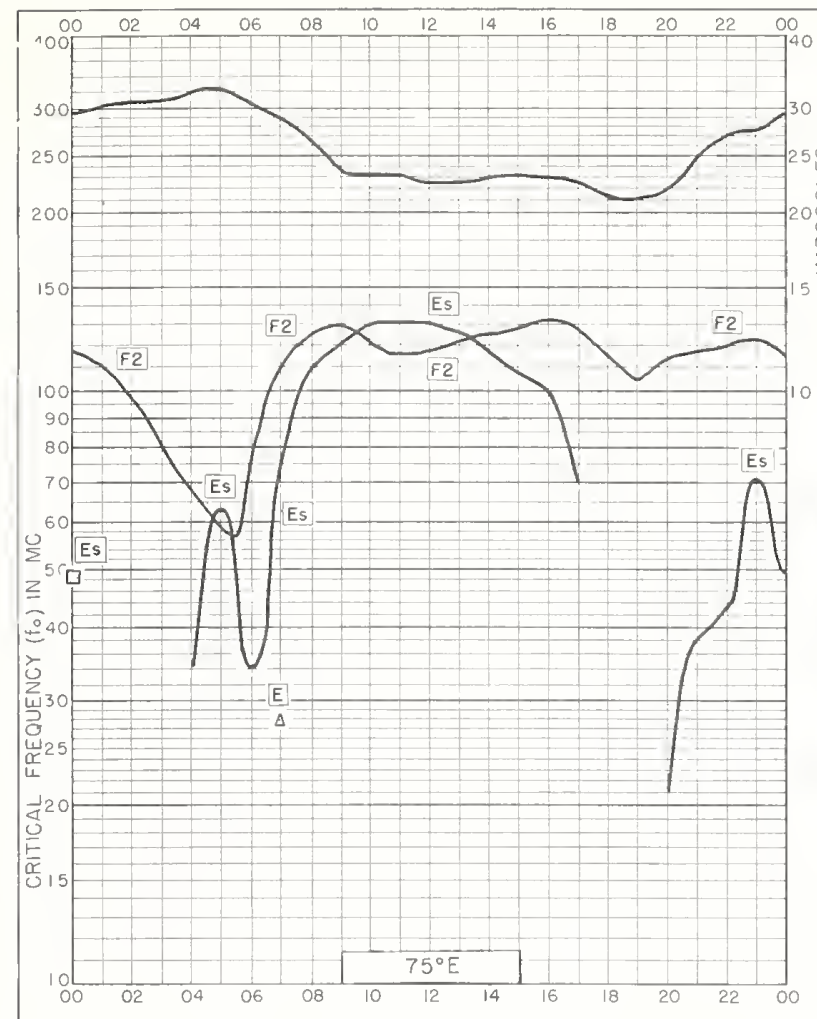

Fig. 61. KODAIKANAL, INDIA 10. $2^{\circ} \mathrm{N}, 77.5^{\circ} \mathrm{E} \quad$ OCTOBER 1959

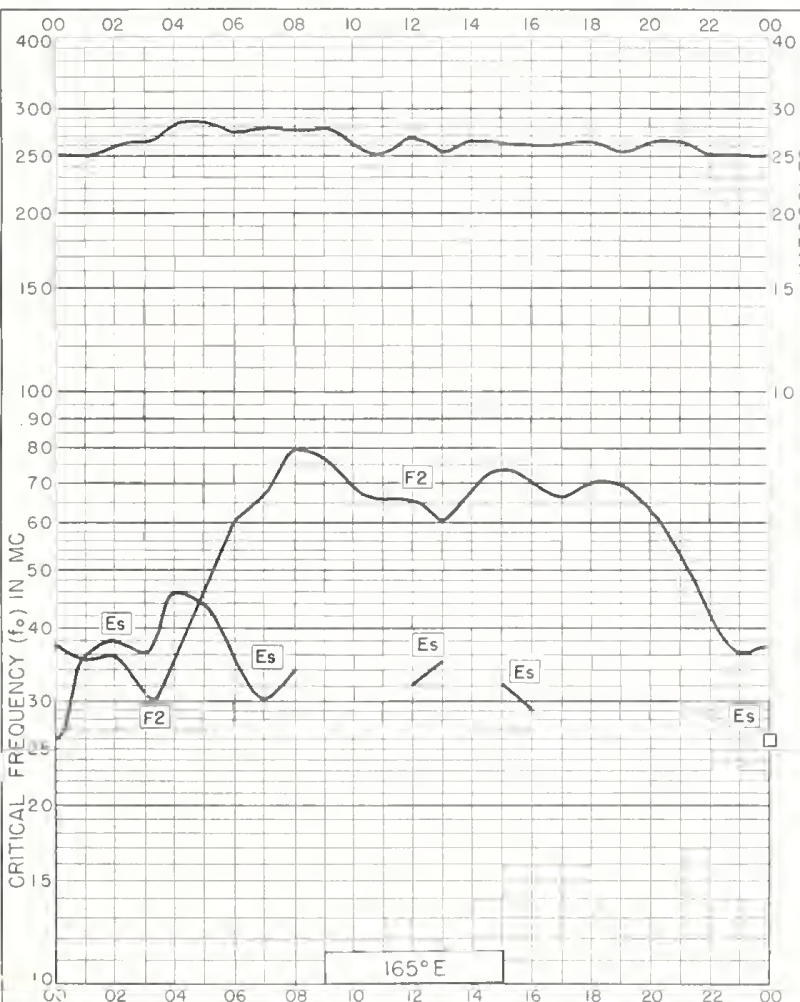

Fig. 63. CAPE HALLETT

$723^{\circ} \mathrm{S}, 170.2^{\circ} \mathrm{E}$ OCTOBER 1959
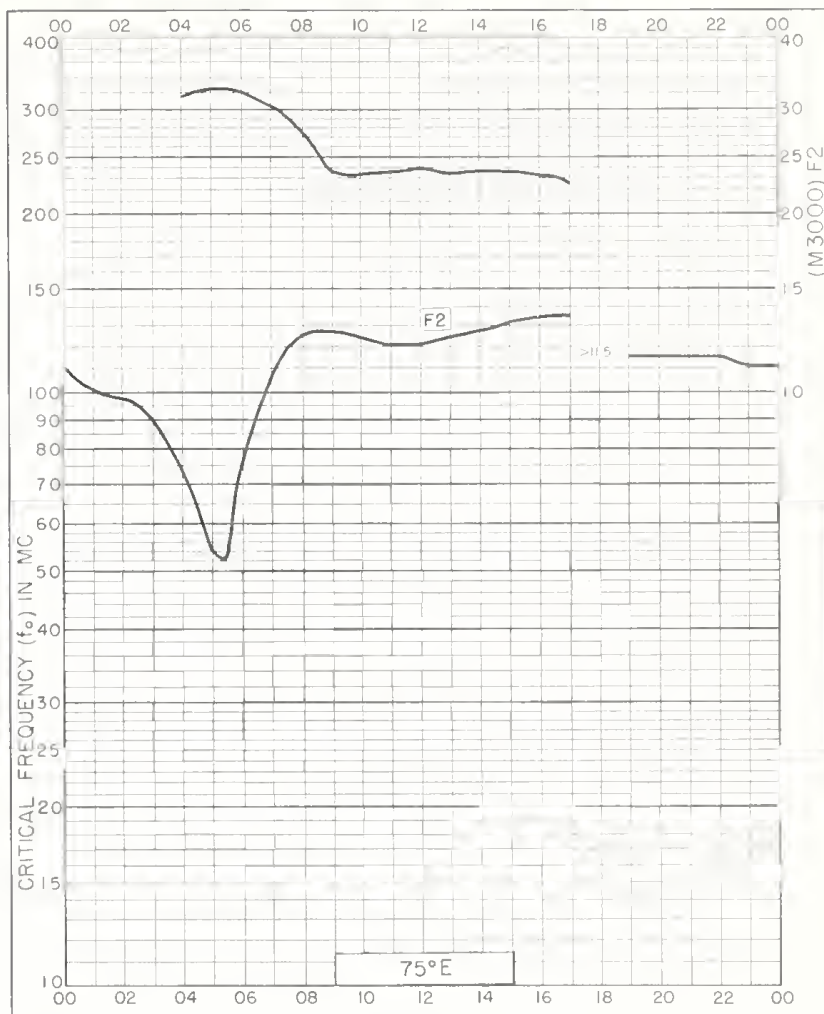

Fig. 62. TRIVANDRUM, INDIA 8. $5^{\circ} \mathrm{N}, 77.0^{\circ} \mathrm{E}$

OCTOBER 1959

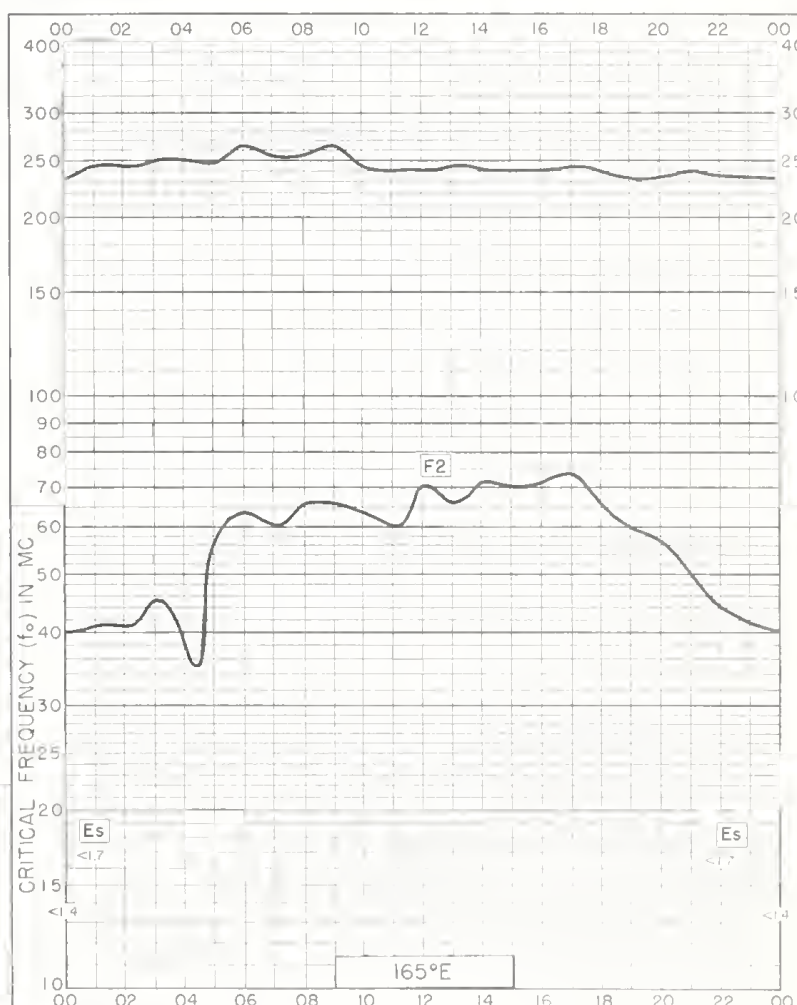

Fig. 64. SCOTT BASE

77. $9^{\circ} \mathrm{S}, 166.8^{\circ} \mathrm{E} \quad$ OCTOBER 1959 


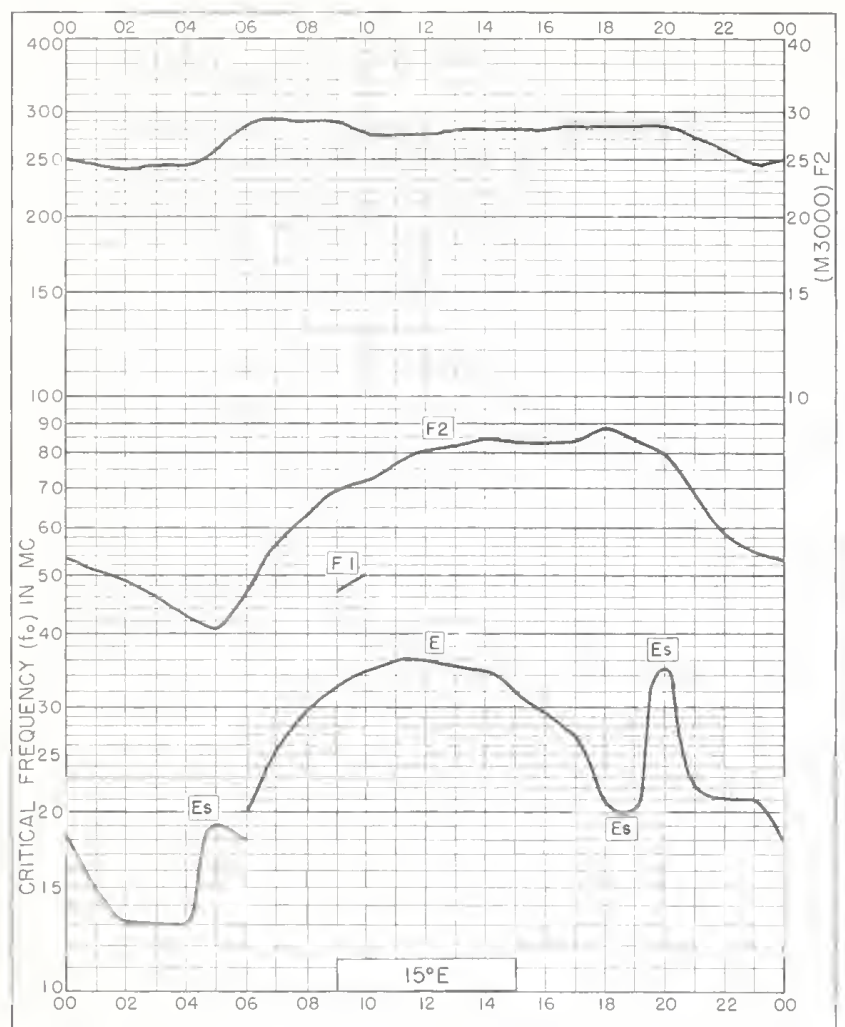

Fig. 65. JULIUSRUH/RUGEN, GERMANY $54.6^{\circ} \mathrm{N}, 13.4^{\circ} \mathrm{E} \quad$ SEPTEMBER 1959

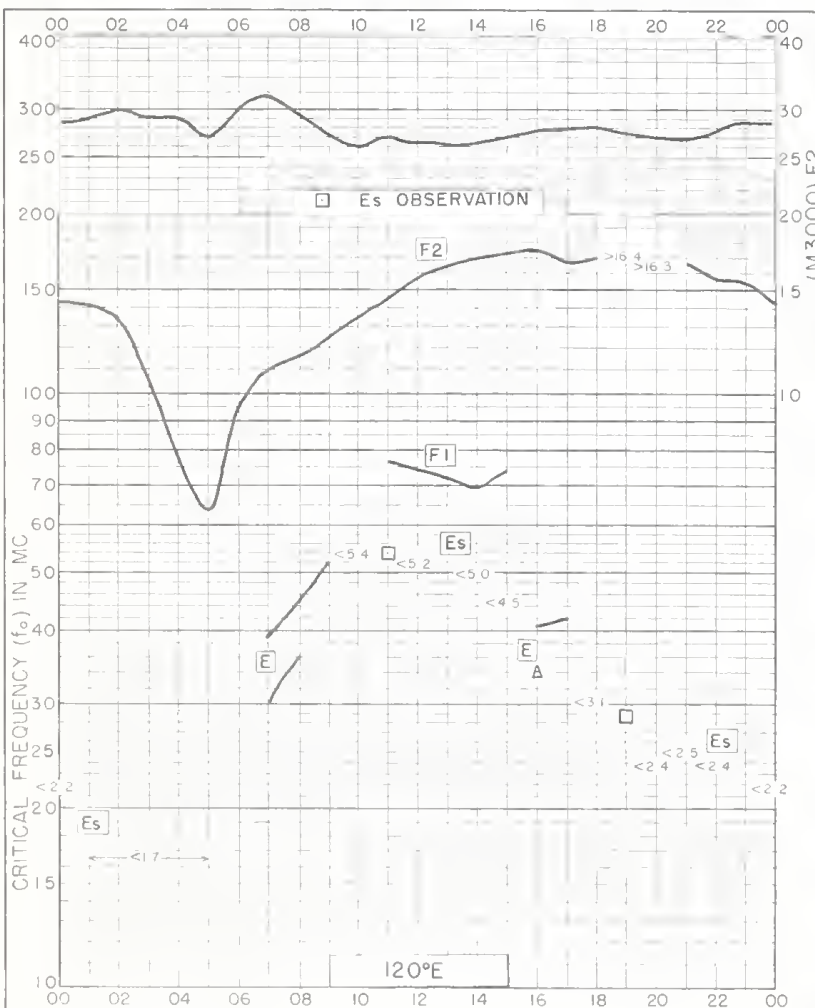

Fig. 67. FORMOSA, CHINA $25.0^{\circ} \mathrm{N}, 121.5^{\circ} \mathrm{E}$ SEPTEMBER 1959

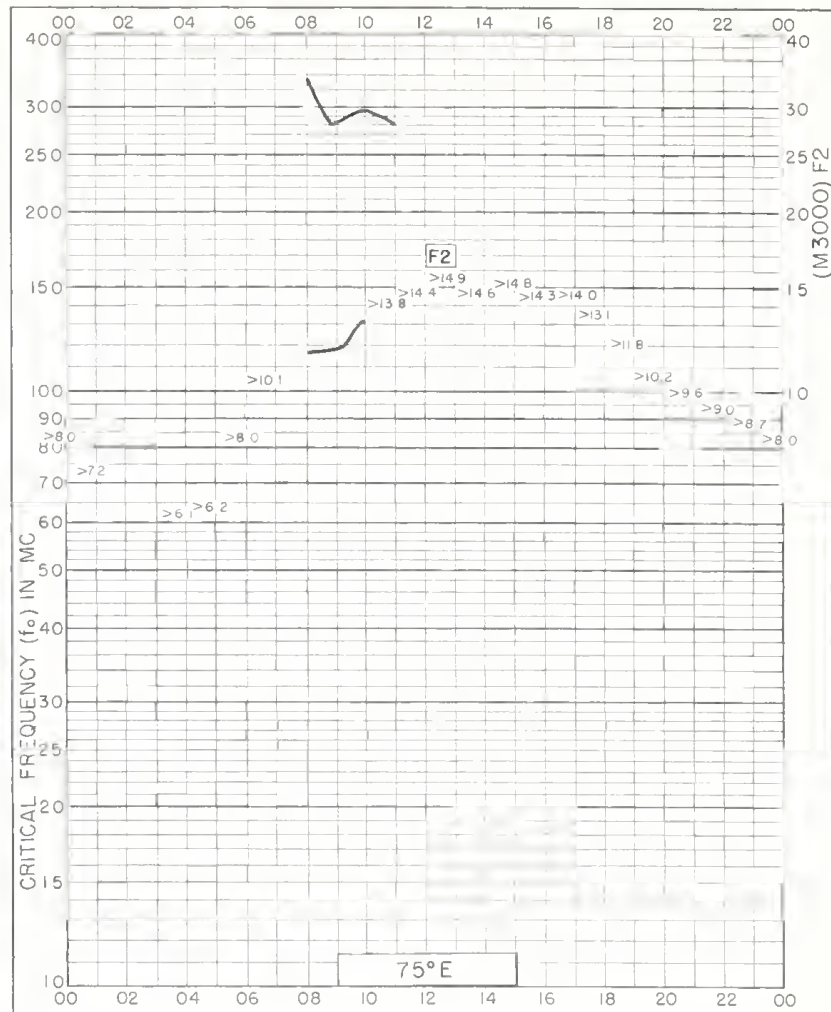

Fig. 66. DELHI, INDIA

$28.6^{\circ} \mathrm{N}, 77.2^{\circ} \mathrm{E}$ SEPTEMBER 1959

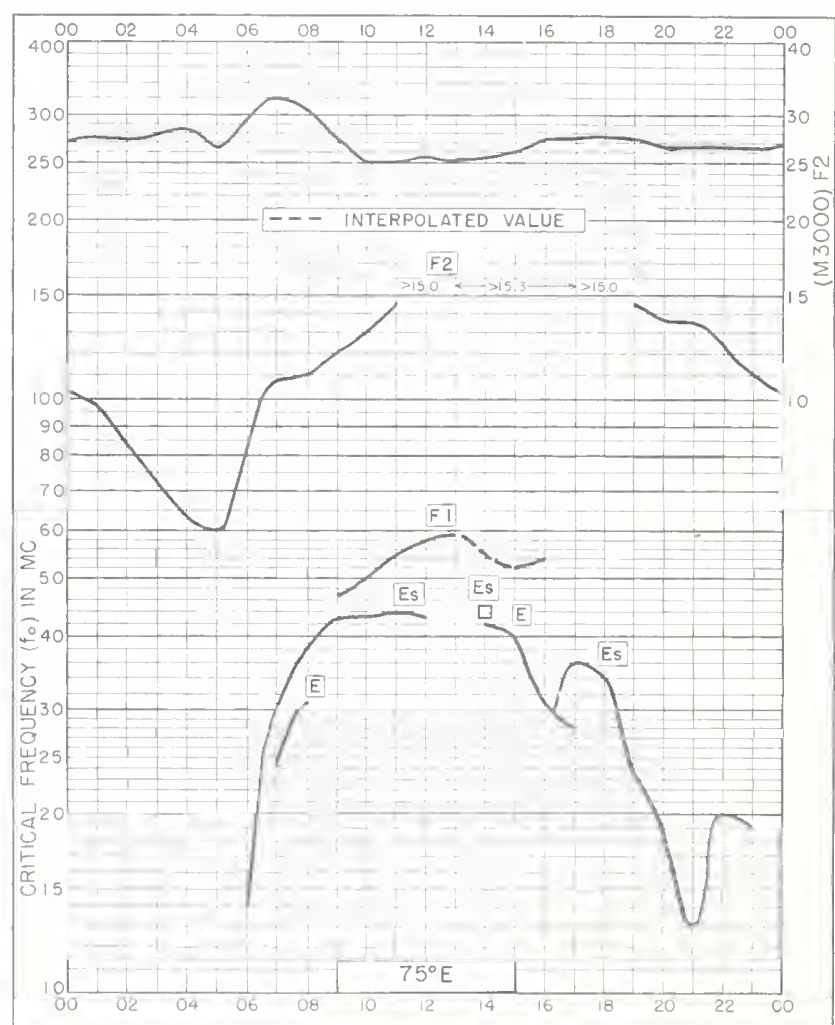

Fig. 68. AHMEDABAD, INDIA $23.0^{\circ} \mathrm{N}, 72.6^{\circ} \mathrm{E} \quad$ SEPTEMBER 1959 

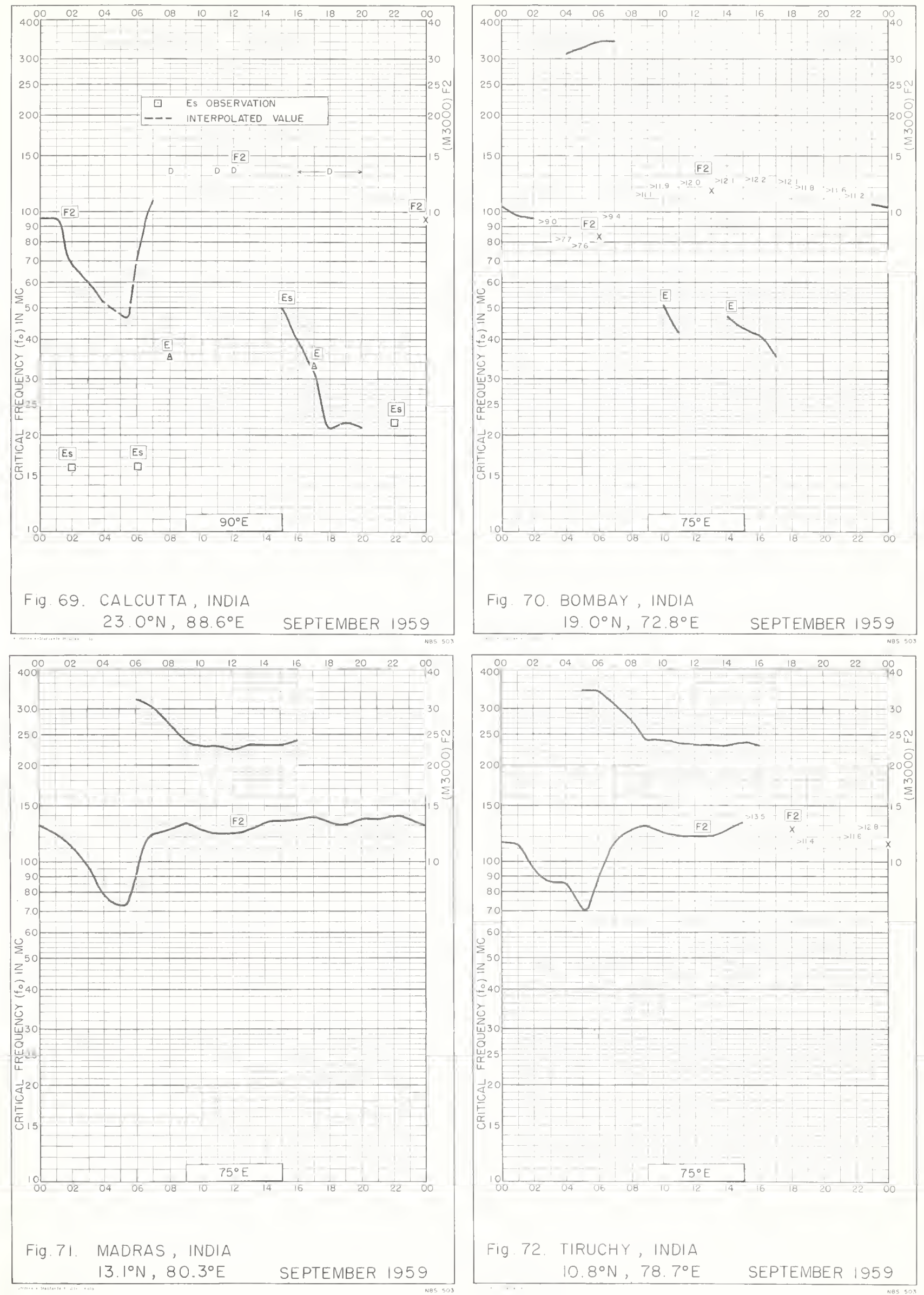
44

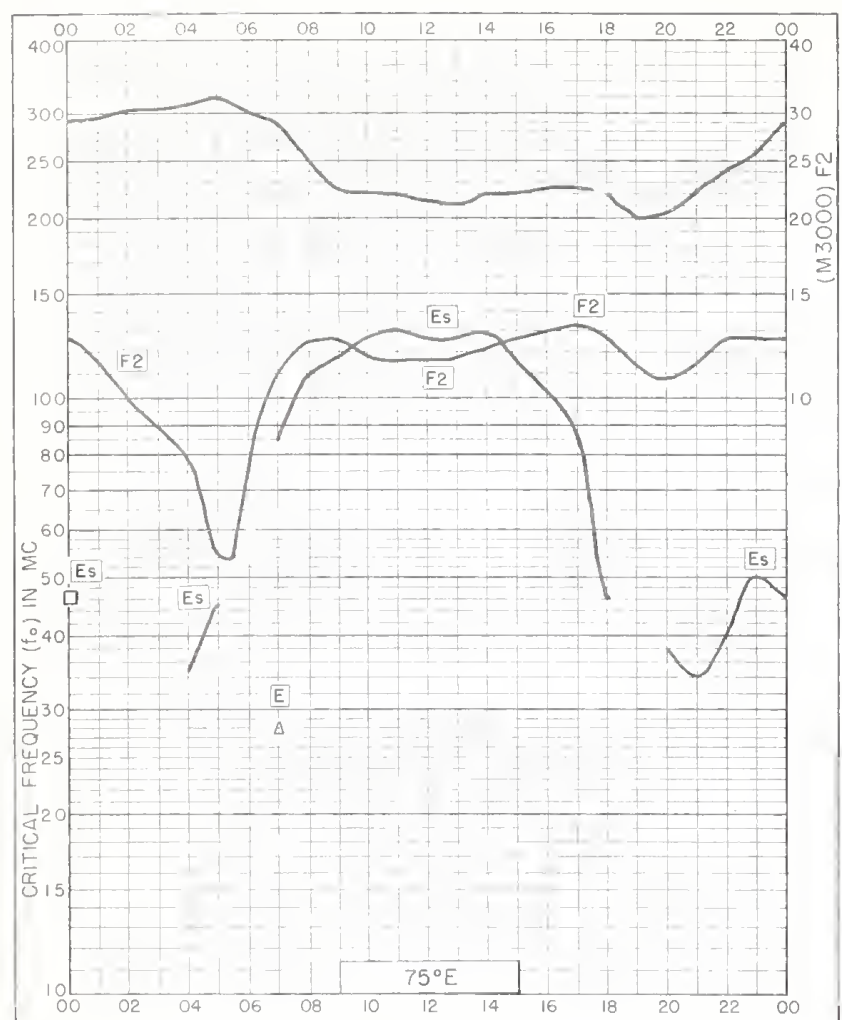

Fig. 73. KODAIKANAL, INDIA

$10.2^{\circ} \mathrm{N}, 77.5^{\circ} \mathrm{E} \quad$ SEPTEMBER 1959

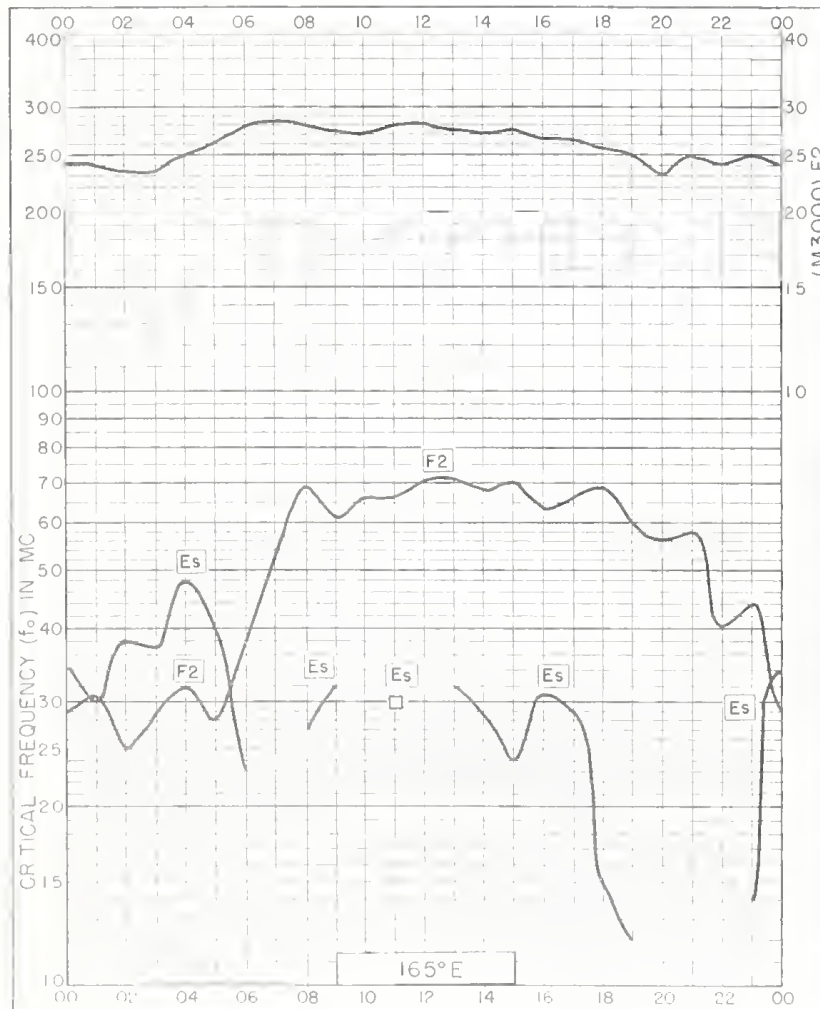

FIg 75. CAPE HALLETT

$72.3^{\circ} \mathrm{S}, 170.2^{\circ} \mathrm{E}$ SEPTEMBER 1959

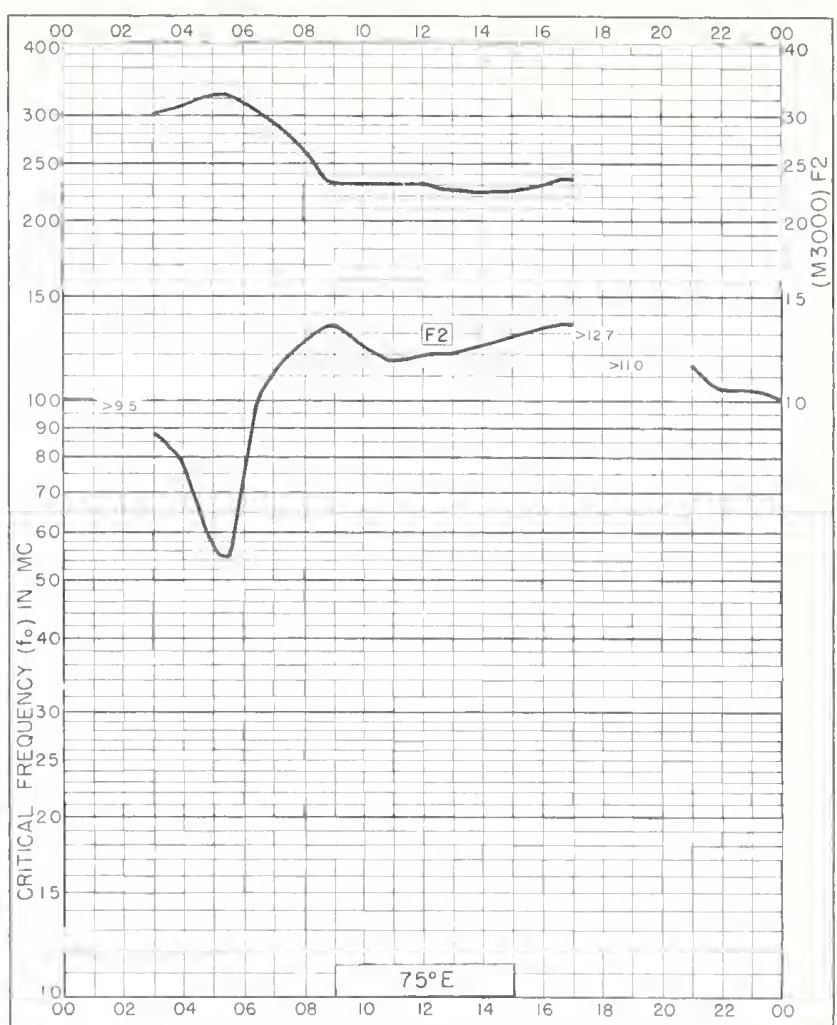

Fig. 74. TRIVANDRUM, INDIA 8. $5^{\circ} \mathrm{N}, 77.0^{\circ} \mathrm{E}$ SEPTEMBER 1959

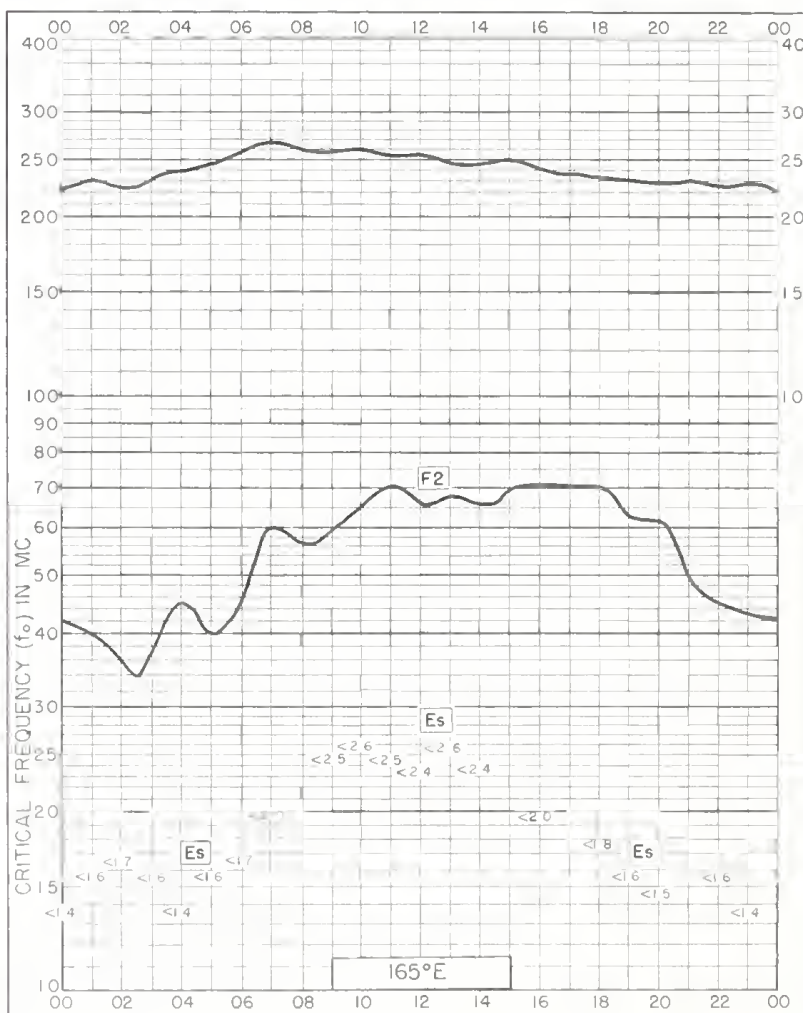

Fig. 76. SCOTT BASE

$77.9^{\circ} \mathrm{S}, 166.8^{\circ} \mathrm{E}$ SEPTEMBER 1959 


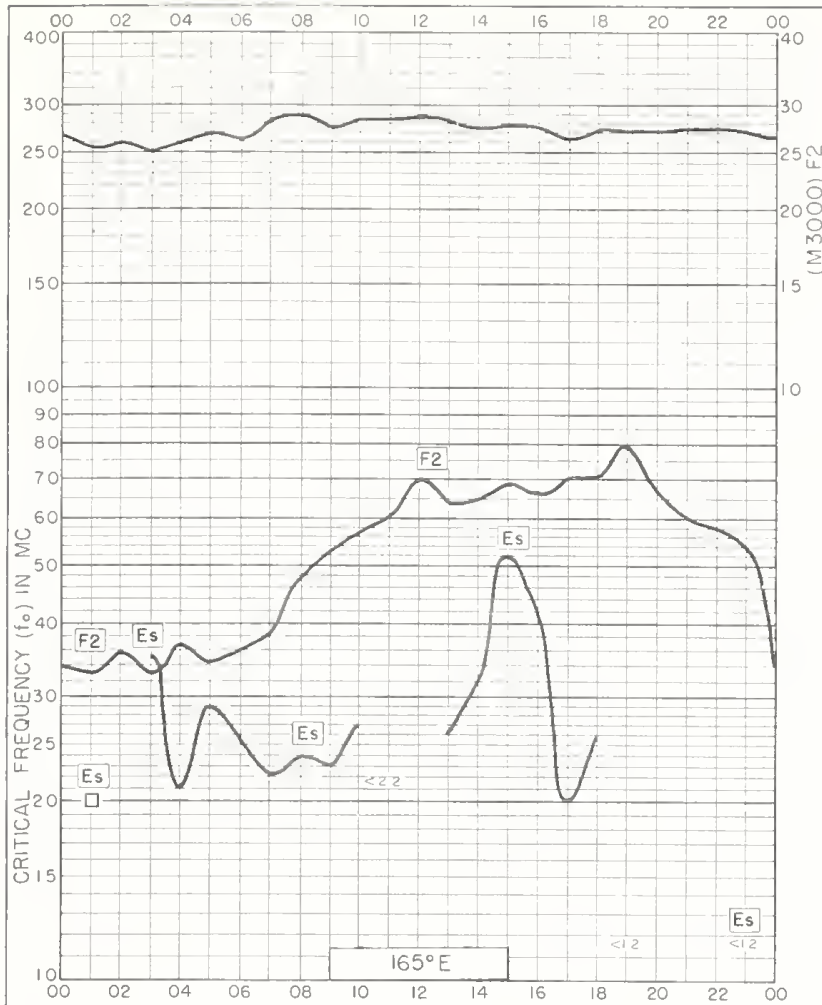

Fig. 77. CAPE HALLETT $72.3^{\circ} \mathrm{S}, 170.2^{\circ} \mathrm{E}$ AUGUST 1959

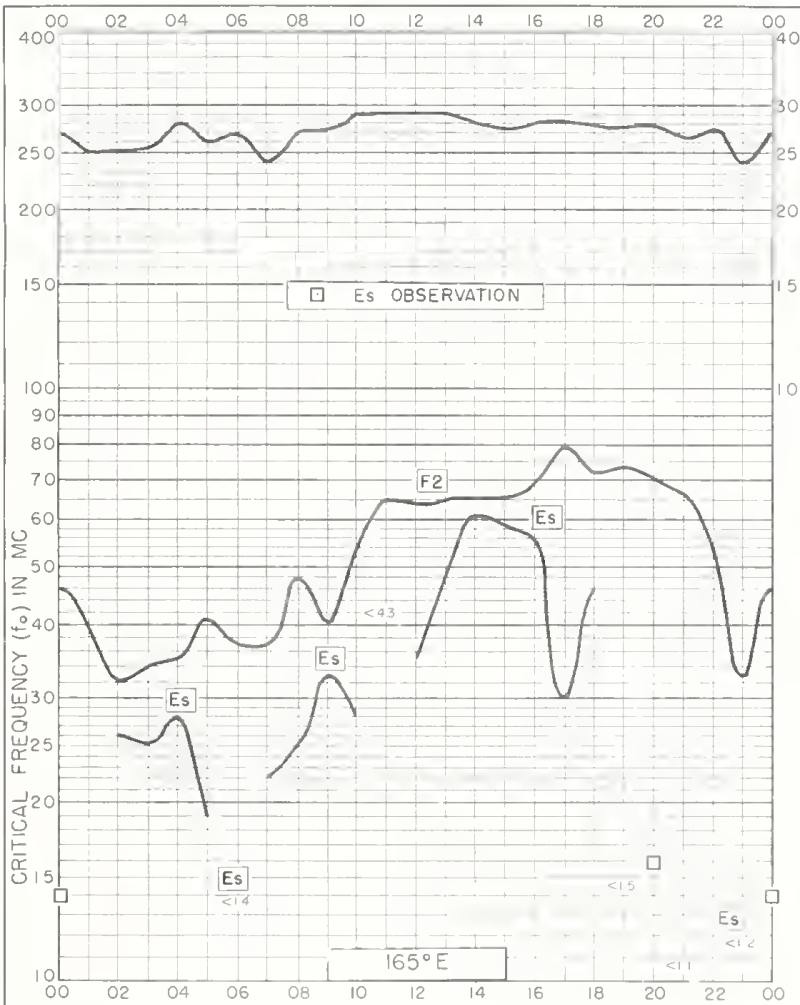

Fig. 79. CAPE HALLETT

$72.3^{\circ} \mathrm{S}, 170.2^{\circ} \mathrm{E}$ JULY 1959

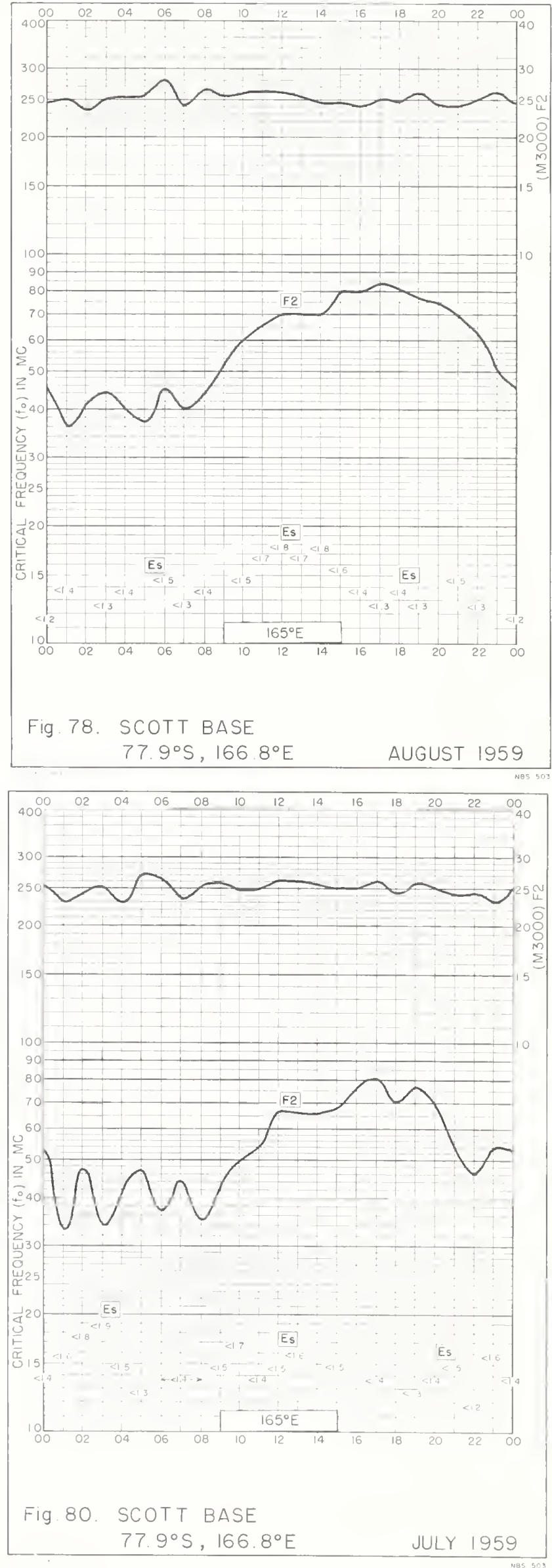




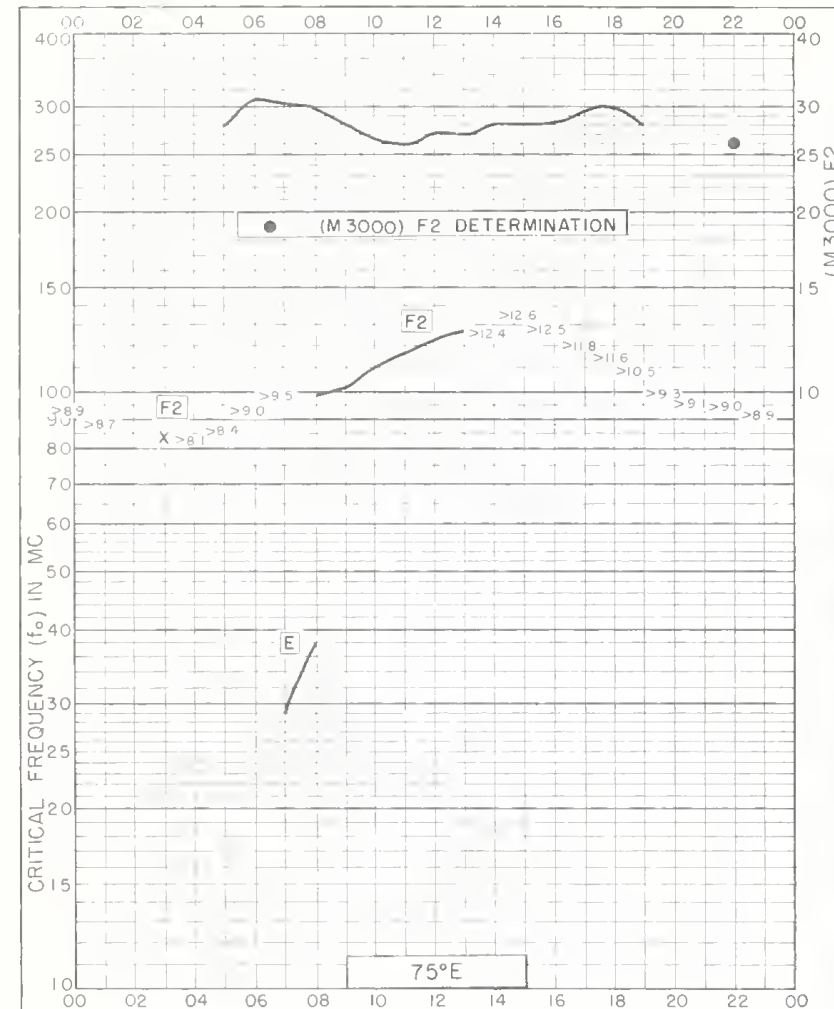

Fig. 8I. DELHI, INDIA

$$
28.6^{\circ} \mathrm{N}, 77.2^{\circ} \mathrm{E}
$$

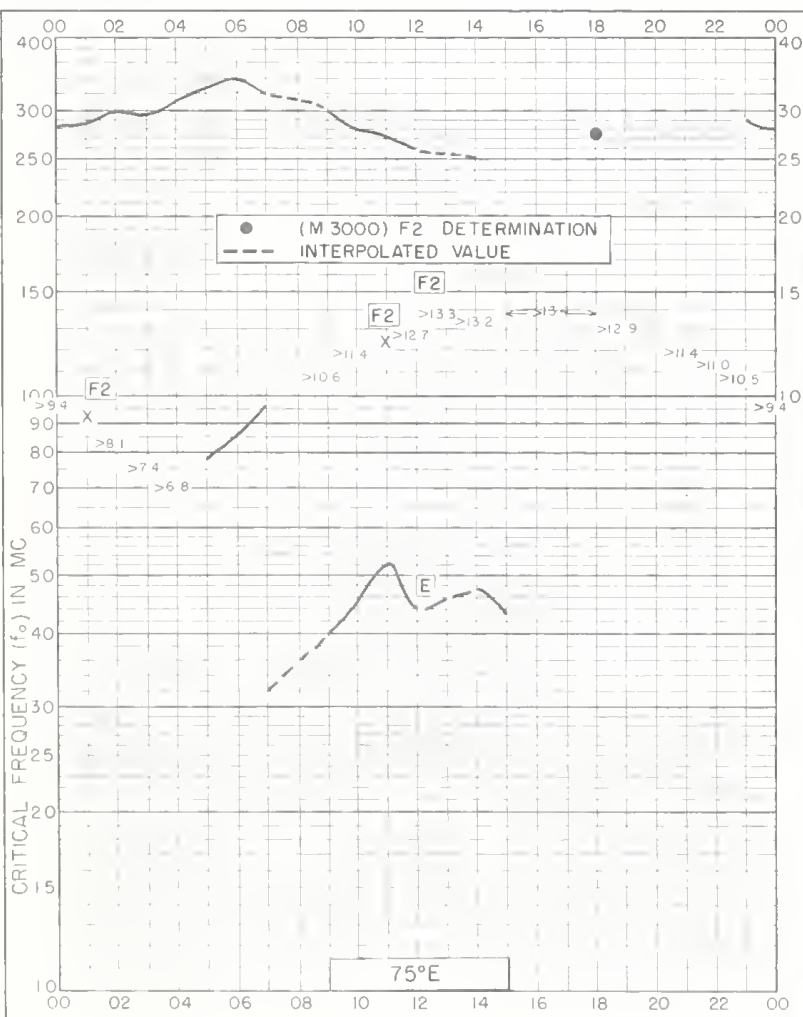

Fig. 83. BOMBAY, INDIA $19.0^{\circ} \mathrm{N}, 72.8^{\circ} \mathrm{E}$
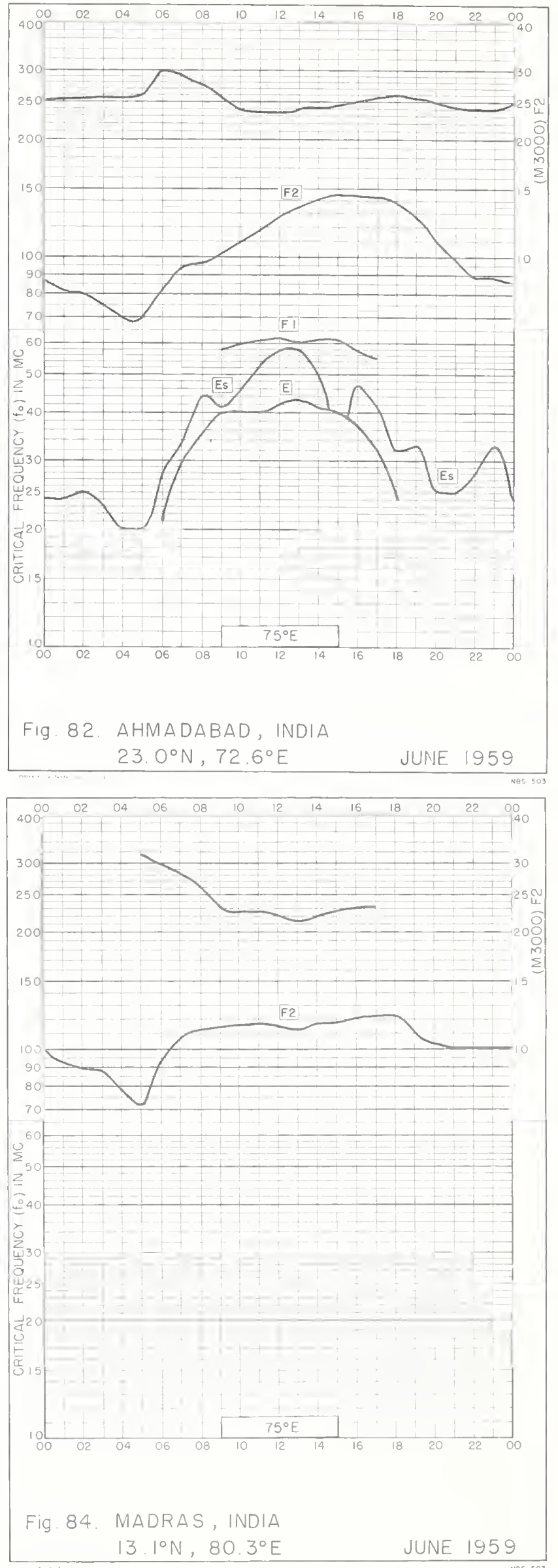


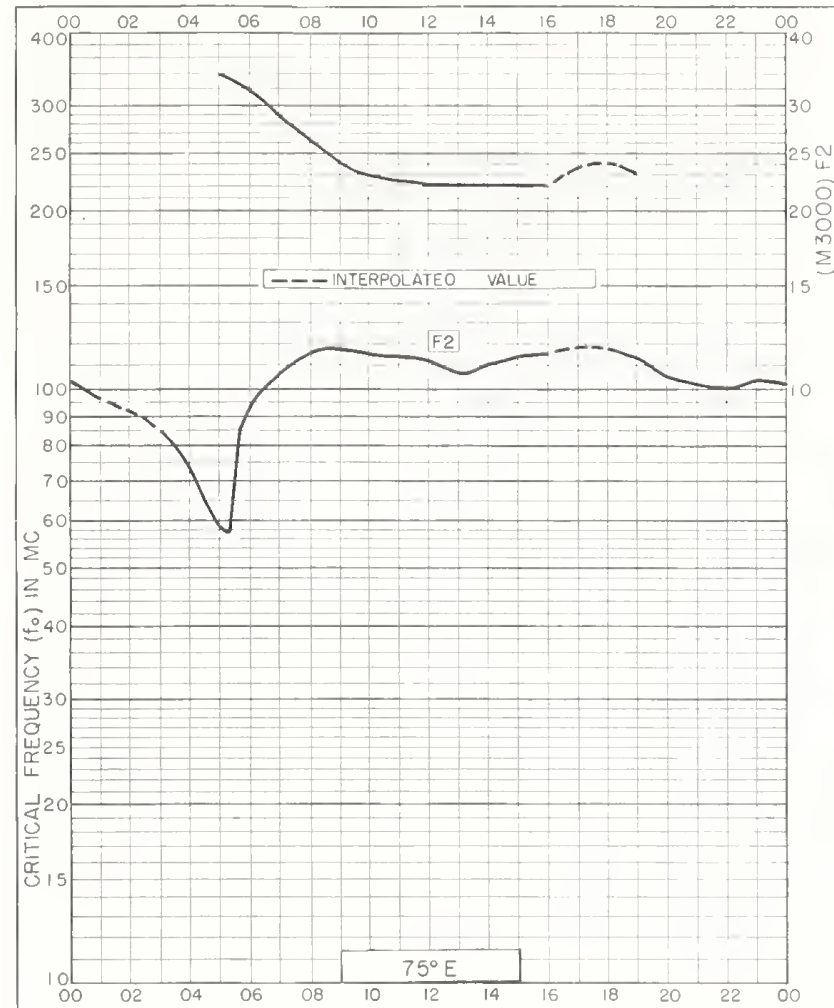

Fig. 85. TIRUCHY, INDIA 10. $8^{\circ} \mathrm{N}, 78.7^{\circ} \mathrm{E}$ JUNE 1959

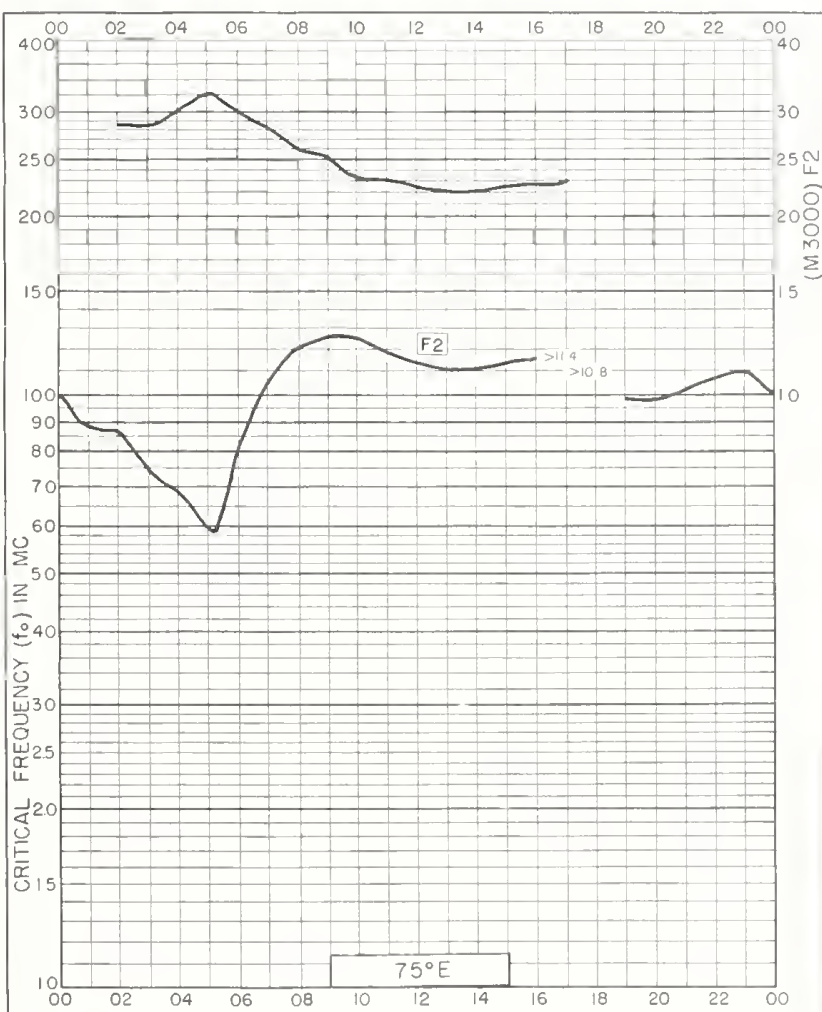

Fig. 87. TRIVANDRUM, INDIA 8. $5^{\circ} \mathrm{N}, 77.0^{\circ} \mathrm{E}$

JUNE 1959

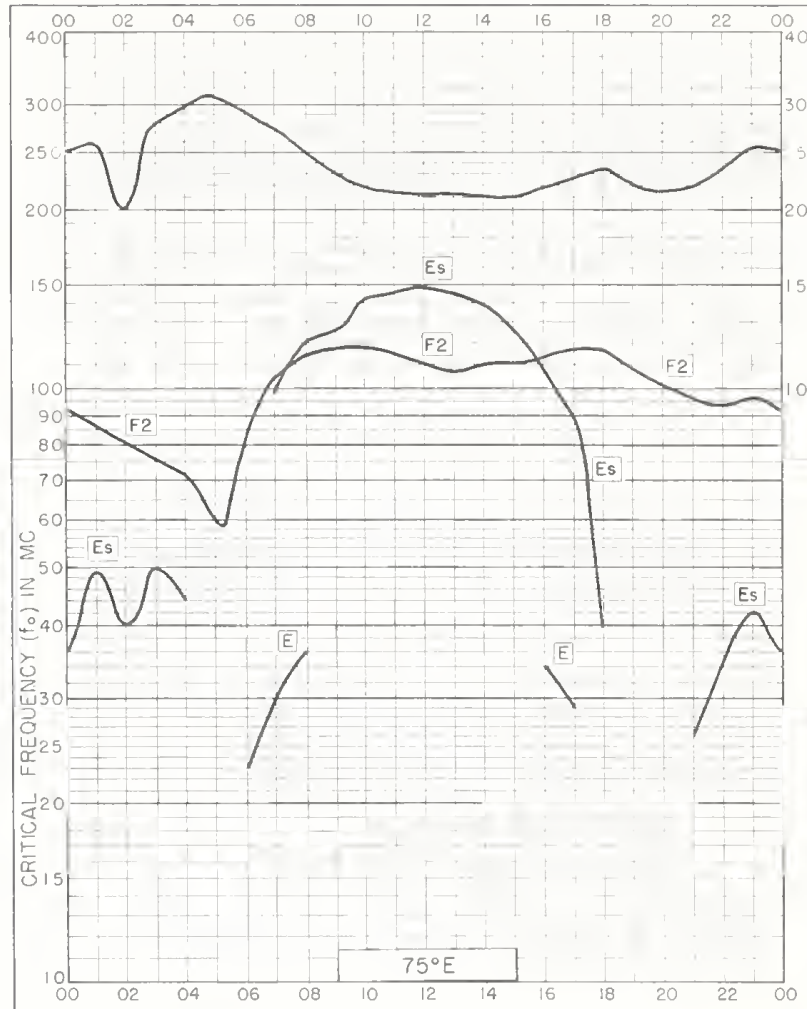

Fig. 86. KODAIKANAL, INDIA 10. $2^{\circ} \mathrm{N}, 77.5^{\circ} \mathrm{E}$

JUNE 1959

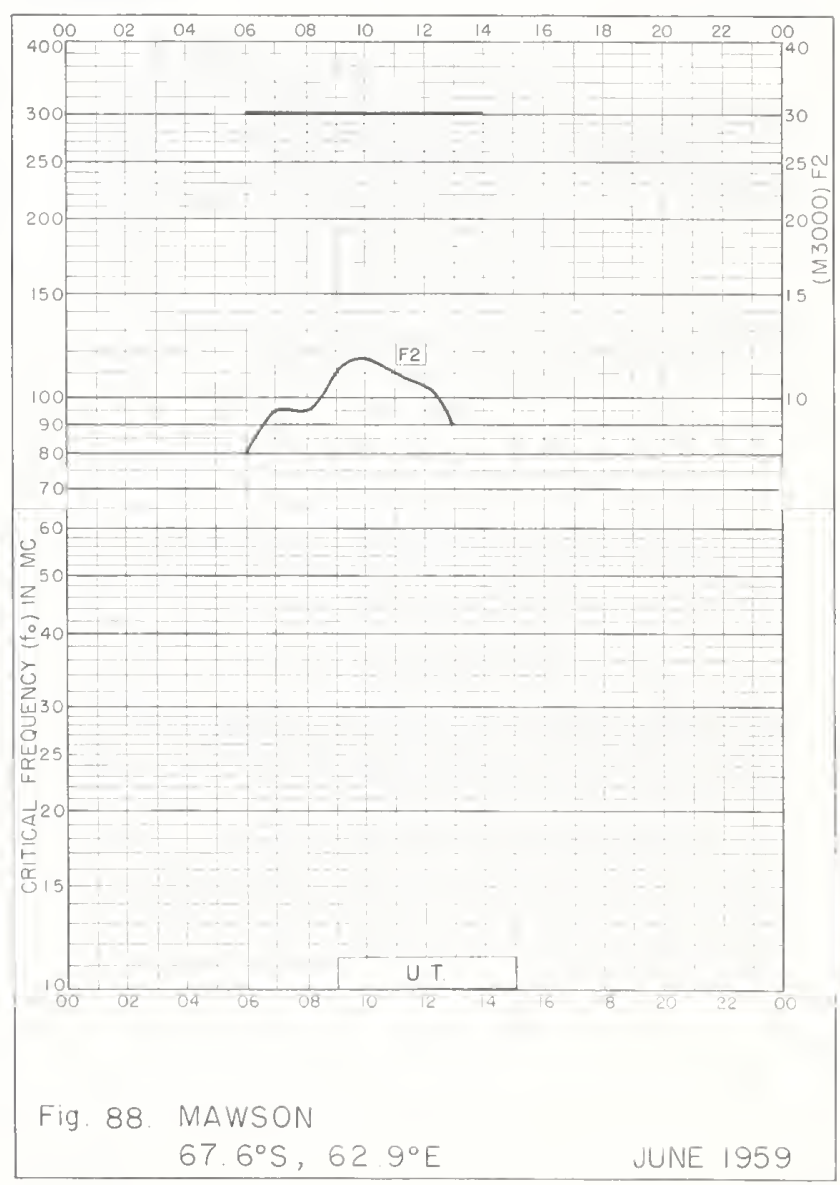




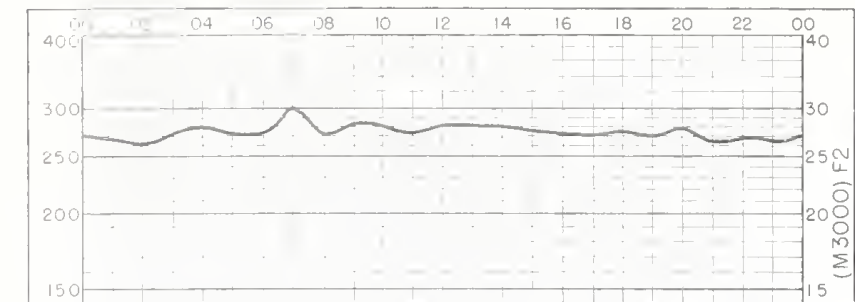

Fig. 89 CAPE HALLETT $723^{\circ} \mathrm{S}, 170.2^{\circ} \mathrm{E}$

JUNE 1959

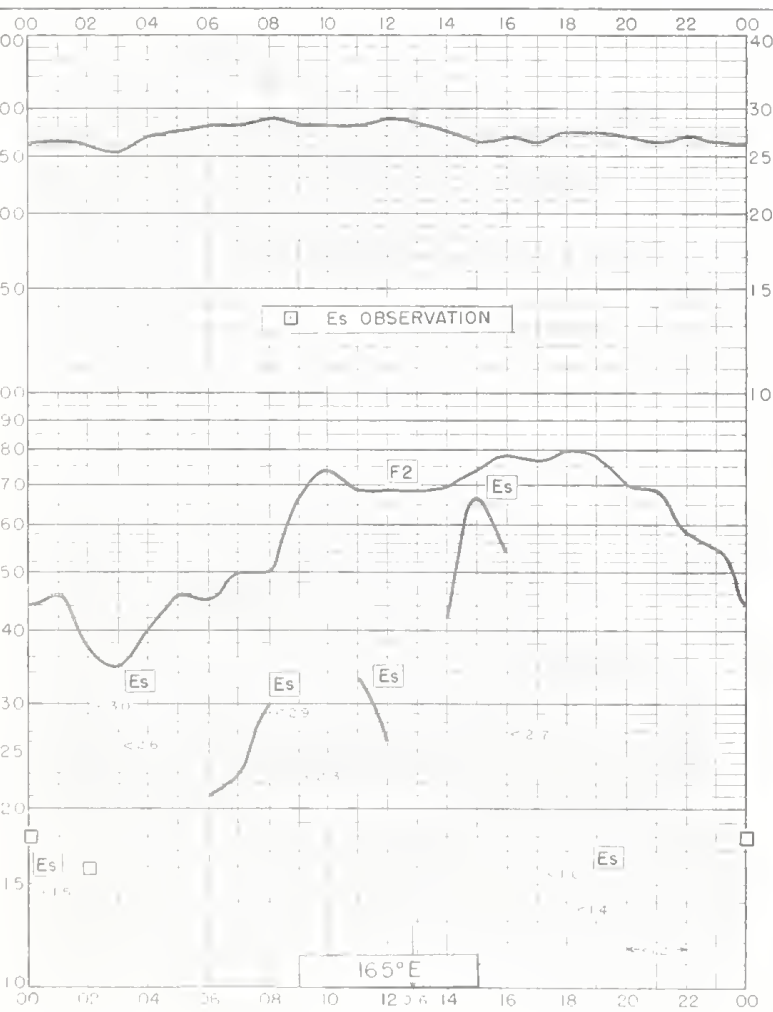

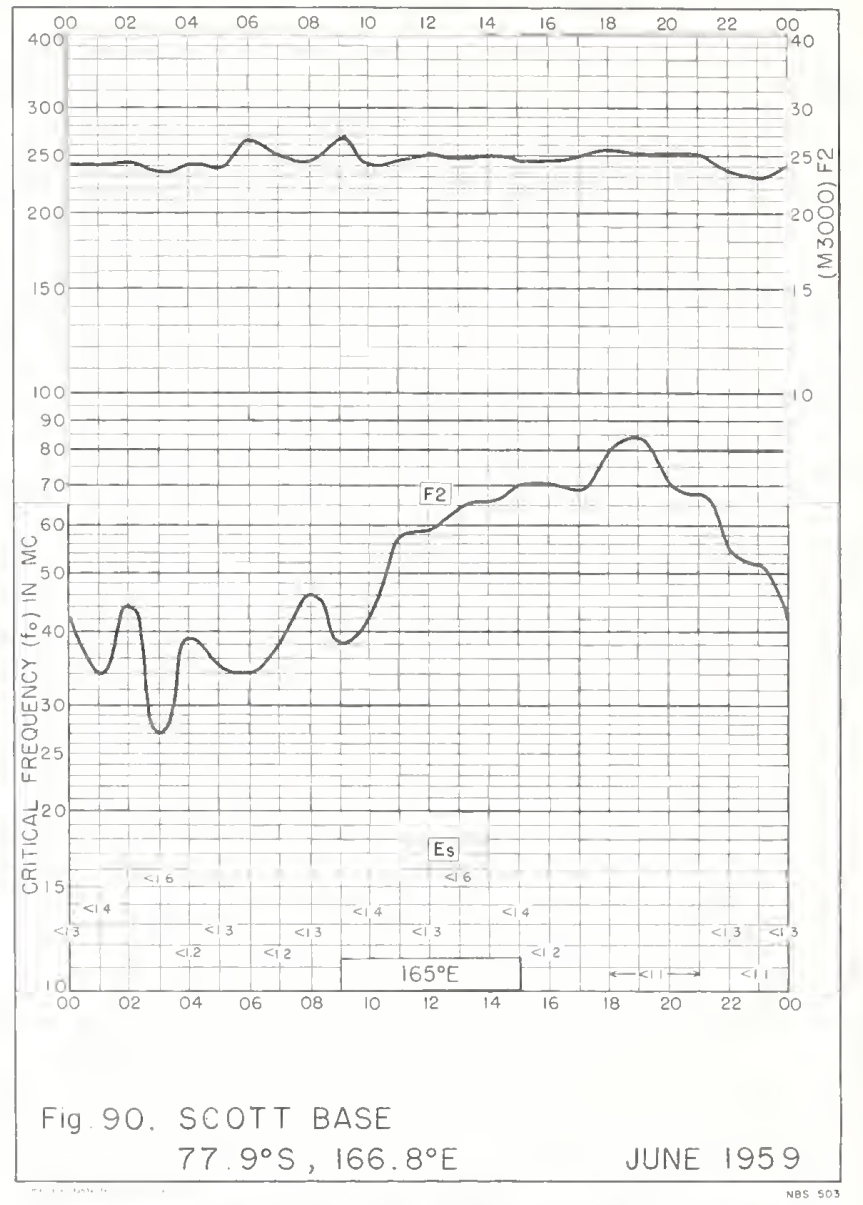

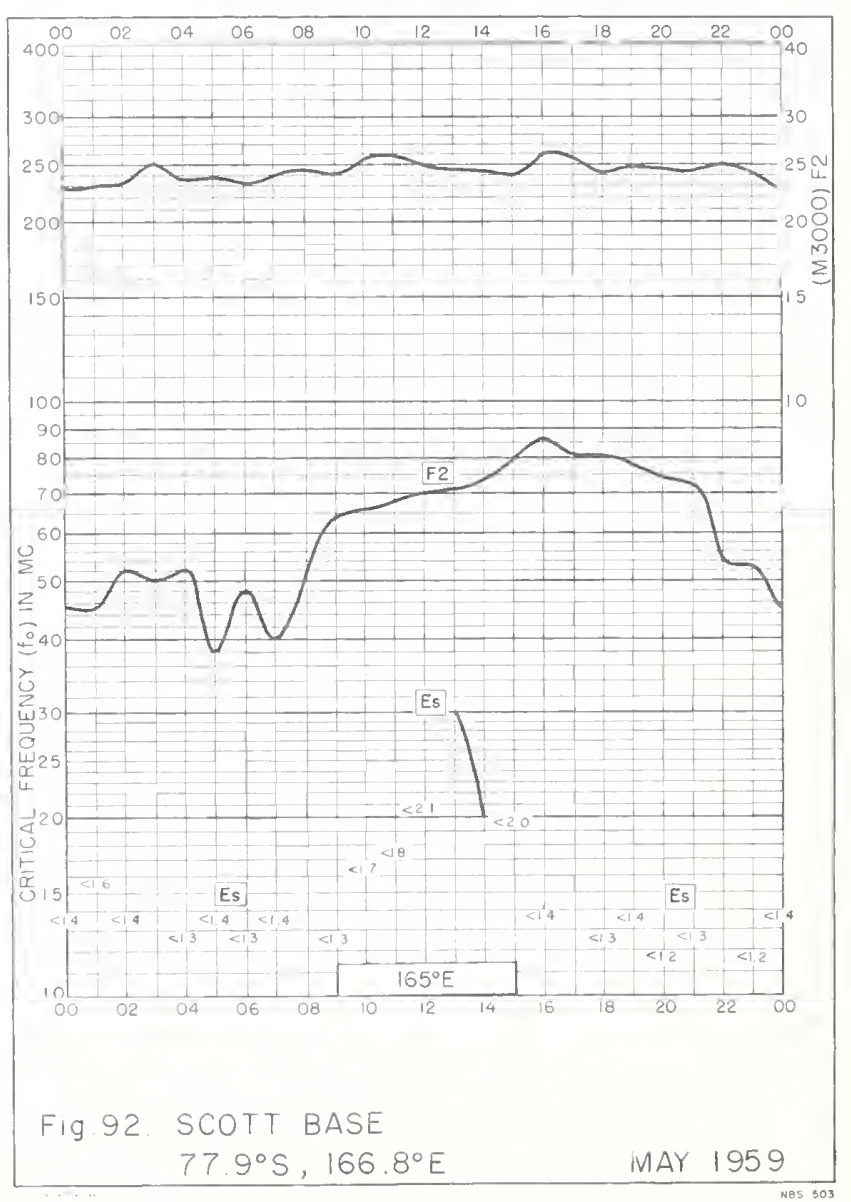




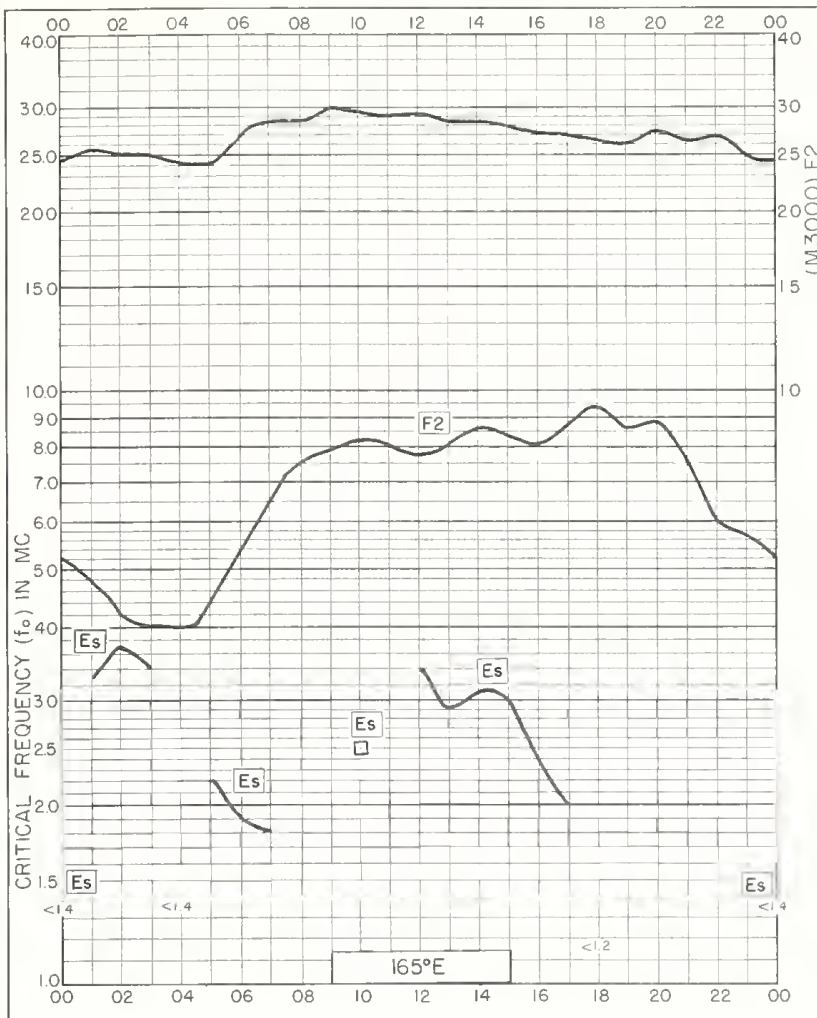

Fig. 93. CAPE HALLETT

$72.3^{\circ} \mathrm{S}, 170.2^{\circ} \mathrm{E}$

APRIL 1959

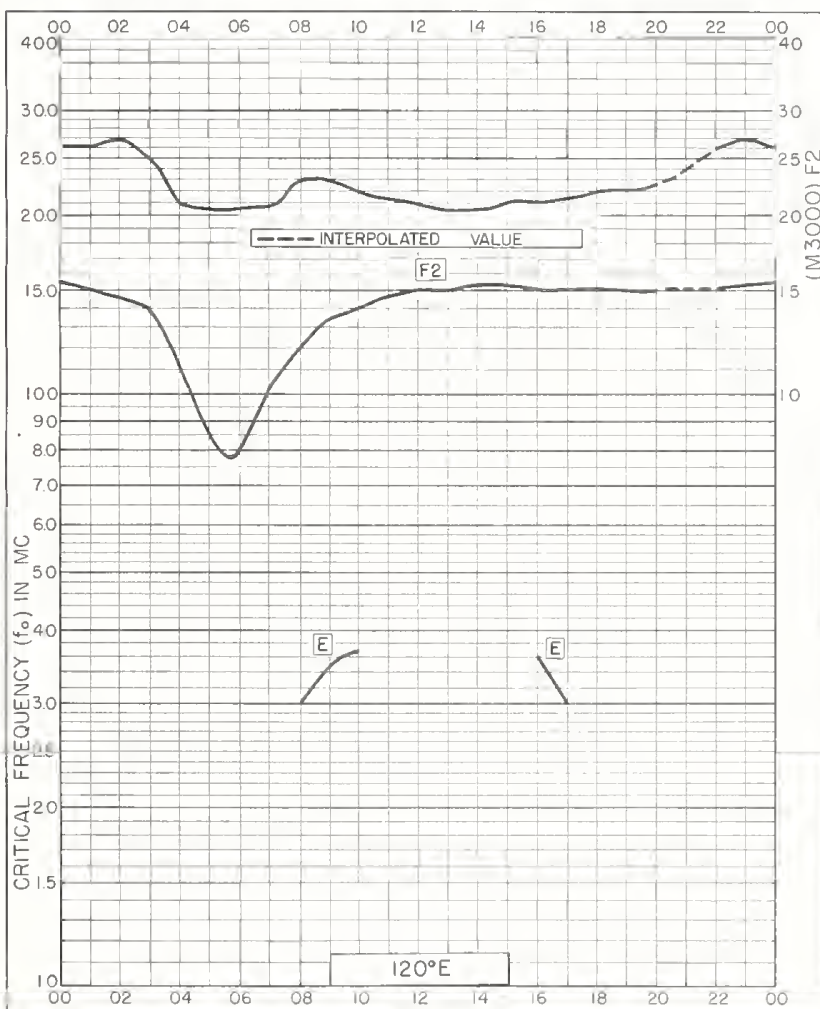

Fig. 95. MACAU $22.2^{\circ} \mathrm{N}, 113.6^{\circ} \mathrm{E}$

MARCH 1959
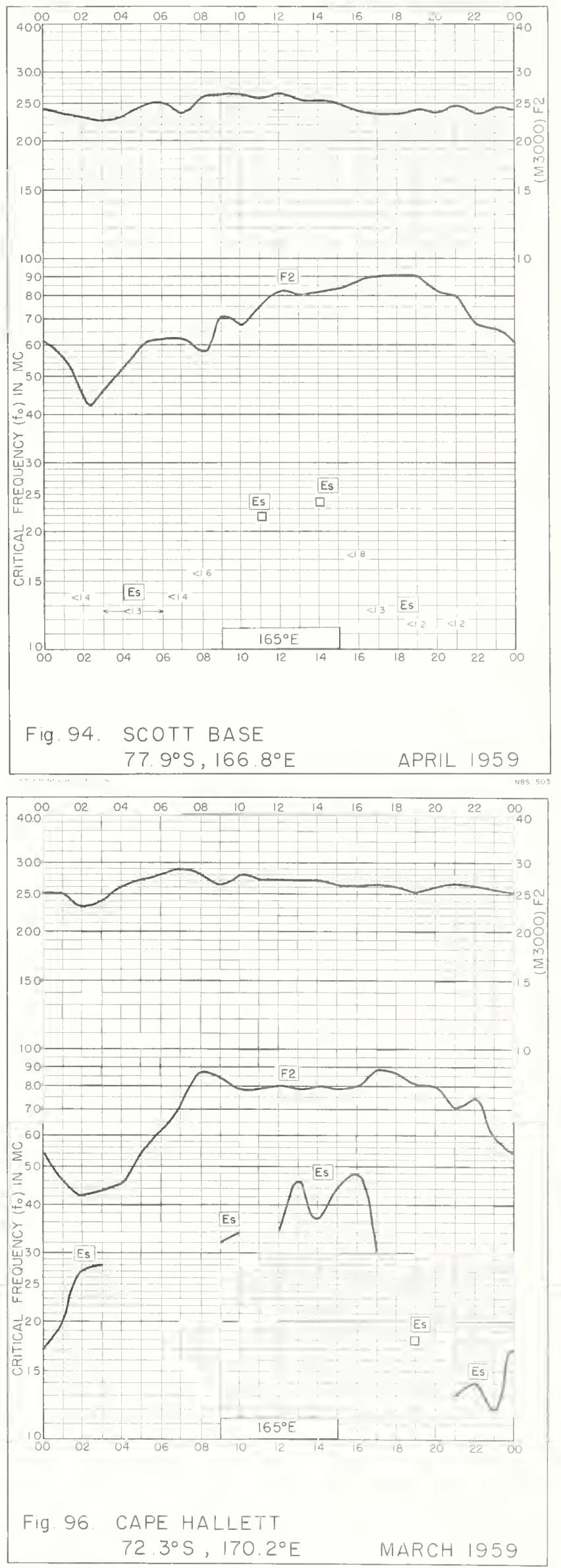

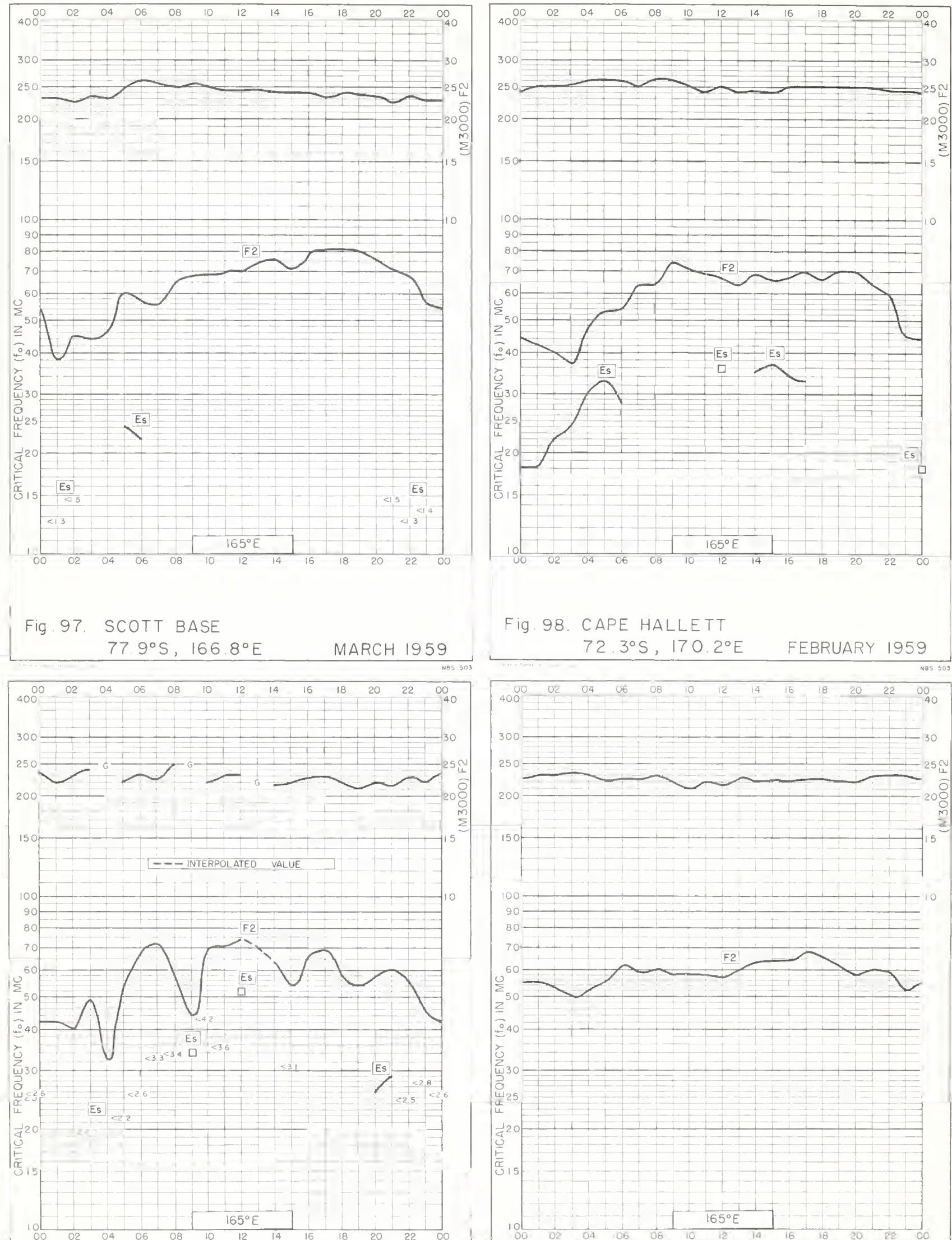


\section{INDEX OF IONOSPHERIC DATA IN CRPL F220}

\section{PAGE \\ TABLE FIGURE}

AHMEDABAD, INDIA

BOMBAY, INDIA

BRISBANE, AUSTRALIA

CALCUTTA, INDIA

CANBERRA, AUSTRALIA

CAPE HALLETT

DELHI, INDIA

$\begin{array}{ll}1959 & \text { JUNE } \\ 1959 & \text { SEPT. } \\ 1959 & \text { OCT. } \\ 1959 & \text { NOV. } \\ 1959 & \text { DEC. }\end{array}$

1959 JUNE

1959 SEPT。

1959 OCT.

1959 NOV.

1959 DEC.

1962 JAN.

1959 SEPT.

1959 OCT.

1959 NOV.

1959 DEC.

1962 JAN.

1959 FEB.

1959 MAR。

1959 APR。

1959 MAY

1959 JUNE

1959 JULY

1959 AUG.

1959 SEPT。

1959 OCT.

1959 NOV.

1959 DEC。

$\begin{array}{rr}21 & 46 \\ 17 & 42 \\ 14 & 39 \\ 12 & 37 \\ 9 & 34\end{array}$

$21 \quad 46$

1843

1540

$12 \quad 37$

$10 \quad 35$

$2 \quad 27$

1843

1540

$12 \quad 37$

$103 b$

$2 \quad 27$

$\begin{array}{ll}25 & 50 \\ 24 & 49 \\ 24 & 49 \\ 23 & 48 \\ 23 & 48 \\ 20 & 45 \\ 20 & 45 \\ 19 & 44 \\ 16 & 41 \\ 14 & 39 \\ 11 & 36\end{array}$

1959 JUNE

1959 SEPT.

1959 OCT.

1959 NOV.

1959 DEC.

FAIRBANKS, ALASKA

1961 AUG.

530

FORMOSA, CHINA

1959 SEPT.

$17 \quad 42$ 
INDEX OF IONOSPHERIC DATA IN CRPL F220

TABLE FIGURE

FT. MONMOUTH, NEW JERSEY

GRAZ, AUSTRIA

HOBART, TASMANIA

JULIUSRUH/RUGEN, GERMANY

KODAIKANAL, INDIA

LYCKSELE, SWEDEN

MACAU

MADRAS, INDIA

MAUI, HAWAII

MAWSON
1961 NOV.

1961 DEC.

$1959 \mathrm{DEC}$.

$9 \quad 34$

1962 JAN。

$2 \quad 27$

1959 SEPT.
1959 DEC.

$17 \quad 42$

$8 \quad 33$

1959 JUIVE

1959 SEPT.

1959 OCT .

1959 NOV.

1959 DEC.

1959 DEC.

$22 \quad 47$

$19 \quad 44$

1641

$13 \quad 38$

1136

1959 MAR.

$24 \quad 49$

1959

1959

1959

1959

1959

JUNE

SEPT •

OCT.

NOV.

DEC。

1961 MAY

1961 JUNE

1961 JULY

1961 AUG.

1961 SEPT.

1961 OCT.

1961 NOV.

1961 DEC.

1962 JAN.

1962 FEB.

1962 MAR.

1959 JUNE

2146

1843

1540

$13 \quad 38$

$10 \quad 35$
631

631

530

530

$4 \quad 29$

$4 \quad 29$

328

328

126

126

126

$22 \quad 47$ 
INDEX OF IONOSPHERIC DATA IN CRPL F220

PAGE
TABLE FIGURE

REYKJAVIK, ICELAND

SCOTT BASE

SOTTENS, SWITZERLAND

ST. JOHNS. NEWFOUNDLAND

THULE, GREENLAND

TIRUCHY, INDIA

TRIVANDRUM, INDIA

WASHINGTON, D.C.

WHITE SANDS, NEW MEXICO

$\begin{array}{ll}1961 & \text { JAN. } \\ 1961 & \text { FEB. } \\ 1961 & \text { MAR. } \\ 1961 & \text { APR. } \\ 1961 & \text { MAY } \\ 1961 & \text { JUNE } \\ 1961 & \text { JULY } \\ 1961 & \text { SEPT. } \\ 1961 & \text { OCT. }\end{array}$

1959

1959

1959

1959

1959

1959

1959

1959

1959

1959

1959

1959

1959 DEC.

1959 DEC.

1961 NOV.

1959 JUNE
1959 SEPT.
1959 OCT.
1959 NOV.
1959 DEC.

1959

1959

1959

1959

1959

JUNE
SEPT.
OCT.
NOV.
DEC.

1962 FEB.

$22 \quad 47$

1843

1540

1338

1035

$25 \quad 50$

$\angle 5 \quad 50$

$25 \quad 50$

$24 \quad 49$

$23 \quad 48$

2348

$20 \quad 45$

$20 \quad 45$

1944

1641

1439

1136

833

328

$10 \quad 35$

$22 \quad 47$

1944

1641

1338

1136

126

1961 APR.

$7 \quad 32$ 



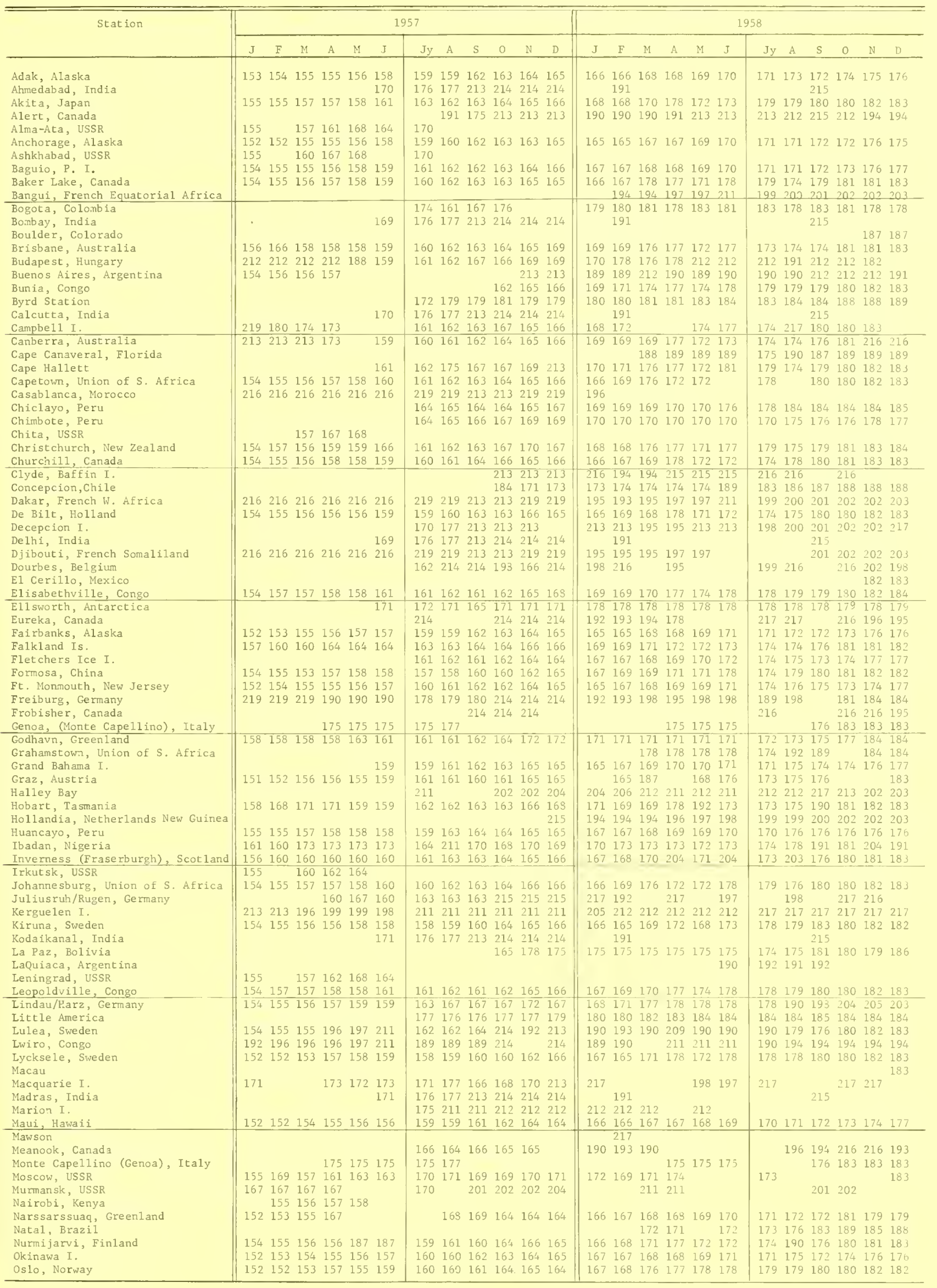




\begin{tabular}{|c|c|c|c|c|c|c|c|c|c|c|c|c|c|c|c|c|c|c|c|c|c|c|c|c|}
\hline \multirow[t]{2}{*}{ Station } & \multicolumn{12}{|c|}{1957} & \multicolumn{12}{|c|}{1958} \\
\hline & J & F & M & $\mathrm{A}$ & $\mathrm{M}$ & $\mathrm{J}$ & Jy & A & s & 0 & $\mathrm{~N}$ & D & $\mathrm{J}$ & $\mathrm{F}$ & M & A & $\mathrm{M}$ & $\mathrm{J}$ & Jy & A & S & 0 & $\mathrm{~N}$ & $\mathrm{D}$ \\
\hline Ottawa, Canada & 154 & 155 & 155 & 157 & 158 & 159 & 160 & 160 & 163 & 164 & 165 & 166 & 166 & 167 & 177 & 208 & 171 & 172 & 174 & 179 & 179 & 180 & 182 & 182 \\
\hline Panama Canal Zone & 153 & 154 & 154 & 155 & 156 & 158 & 160 & 161 & 161 & 162 & 164 & 165 & 166 & 167 & 167 & 168 & 169 & 170 & 170 & 171 & 172 & 173 & 174 & 177 \\
\hline Paramaribo, Surinam & & & & & & & 162 & 166 & 169 & 214 & 213 & 213 & 192 & 193 & 194 & 195 & 197 & 198 & 198 & 203 & 201 & 212 & 202 & 203 \\
\hline Point Barrow, Alasica & 152 & 152 & 154 & 155 & 156 & 158 & 159 & 161 & 162 & 163 & 164 & 165 & 165 & 167 & 167 & 167 & 169 & 170 & 171 & 172 & 172 & 175 & 176 & 177 \\
\hline Poitiers, France & 216 & 216 & 216 & 216 & 216 & 216 & 219 & 219 & 213 & 213 & 219 & 219 & 195 & $199^{\prime}$ & 195 & 197 & 197 & 211 & 199 & 200 & 201 & 202 & 202 & 203 \\
\hline Pole Station & & & & & & & 172 & 173 & 173 & 173 & 171 & 164 & 180 & 180 & 182 & 182 & 183 & 183 & 183 & 183 & 184 & 184 & 190 & 191 \\
\hline Port Lockroy & 158 & 158 & & 196 & 195 & 195 & 219 & 177 & 189 & 190 & 189 & 190 & 189 & 189 & 189 & 195 & 197 & 211 & 199 & 200 & & 205 & 203 & 191 \\
\hline Providenie Bay, USSR & & & 160 & 161 & 168 & & & & & & & & & & & & & & & & & & & \\
\hline Pruhonice, Czechoslovakia & & & & & & & & & & & & & & & & & & & & & & & 217 & 219 \\
\hline Puerto Rico (San Juan), W. I. & 152 & 152 & 154 & 156 & 156 & 156 & 159 & 160 & 162 & 162 & 164 & 165 & 167 & 167 & 168 & 169 & 169 & 170 & 170 & 171 & 172 & 173 & 174 & 177 \\
\hline Rabat, Morocco & & & & & & & & & & & & & & 193 & 195 & 197 & 197 & 211 & 199 & 200 & 201 & 202 & 203 & 203 \\
\hline Rarotonga $I$. & 154 & 169 & 157 & 158 & 160 & 161 & 161 & 162 & 163 & 168 & 170 & 168 & 167 & 169 & 176 & 177 & 177 & 178 & 179 & 178 & 180 & 180 & 181 & 183 \\
\hline Resolute Bay, Canada & 154 & 155 & 155 & 158 & 158 & 159 & 161 & 161 & 163 & 163 & 165 & 165 & 166 & 167 & 168 & 170 & 172 & 172 & 174 & 179 & 179 & 180 & 181 & 182 \\
\hline Reykjavik, Iceland & 154 & 155 & 155 & 156 & 157 & 157 & 159 & 162 & 162 & 163 & 164 & 165 & 167 & 167 & 168 & 170 & 170 & 170 & 171 & 172 & 174 & 174 & 176 & 177 \\
\hline Rome, ltaly & & & & & & & & & & & & 169 & 166 & 173 & 171 & 174 & 172 & 172 & 174 & 179 & 180 & 181 & 181 & 183 \\
\hline Rostov-on-Don, USSR & 155 & & 160 & 167 & 168 & & 170 & & & & & & & & & & & & & & & & & \\
\hline $\begin{array}{l}\text { St. John's, Newfoundland } \\
\text { Salehard, USSR }\end{array}$ & & 154 & 154 & 156 & 157 & 157 & $\begin{array}{l}159 \\
170\end{array}$ & 160 & 161 & 162 & 164 & 165 & 166 & 167 & 167 & 168 & 169 & 170 & 175 & 175 & 172 & 174 & 174 & 177 \\
\hline Salisbury, Southern Rhodesia & & & & & & & & & & & & & & 219 & & 219 & 219 & 219 & 217 & 217 & 219 & 216 & 217 & \\
\hline San Francisco, Californi. & 152 & 153 & 155 & 155 & 156 & 158 & 159 & 161 & 173 & 169 & 170 & 170 & 169 & 170 & 170 & 170 & 171 & 172 & 173 & 173 & 174 & 174 & 177 & 177 \\
\hline $\begin{array}{l}\text { San Juan, Puerto Rico } \\
\text { San Salvador I. }\end{array}$ & 152 & 152 & 154 & 156 & 156 & 156 & 159 & 160 & 162 & 162 & 164 & 165 & 167 & 167 & 168 & 169 & 169 & 170 & 170 & 171 & 172 & 173 & $\begin{array}{l}174 \\
188\end{array}$ & $\begin{array}{l}177 \\
188\end{array}$ \\
\hline Sao Paulo, Brazil & 214 & 214 & 214 & 214 & 214 & 219 & 161 & 162 & 163 & 166 & 170 & 166 & 171 & 171 & 176 & 177 & 173 & 192 & 192 & 194 & 216 & 216 & 216 & 216 \\
\hline Schwarzenburg, Switzerland & 154 & 155 & 156 & 157 & 158 & 159 & 159 & 159 & 160 & 166 & 166 & 165 & 167 & 163 & 169 & 173 & 172 & 172 & 173 & 179 & 179 & 181 & 181 & 183 \\
\hline Scott Base & & & 156 & 158 & 158 & 161 & 160 & 162 & 163 & 167 & 169 & 167 & 166 & 171 & 176 & 177 & 172 & 172 & 178 & 178 & 180 & 181 & 182 & 204 \\
\hline Simferopol, USSR & 157 & & 157 & 161 & & 164 & & & & & & & & & & & & & & & & & & \\
\hline Singapore, British Malaya & 158 & 161 & 160 & 160 & 164 & 164 & 164 & 164 & 163 & 164 & 187 & 189 & 191 & 191 & 189 & 172 & 172 & 173 & 174 & 176 & 176 & 181 & 181 & 182 \\
\hline Slough, England & 156 & 157 & 158 & 160 & 160 & 160 & 161 & 162 & 167 & 164 & 166 & 169 & 168 & 171 & 169 & 170 & 172 & 173 & 174 & 203 & 179 & 180 & 183 & 183 \\
\hline Sodankyla, Finland & & & & & & & & 162 & 163 & 168 & 168 & 168 & 167 & 168 & 171 & 171 & 172 & 178 & 173 & 179 & 180 & 180 & 182 & 182 \\
\hline Sottens, Switzerland & 154 & 155 & 156 & 157 & 158 & 159 & 159 & 159 & 160 & 166 & 166 & 165 & 167 & 168 & 169 & 173 & 172 & 172 & 173 & 179 & 179 & 181 & 181 & 183 \\
\hline Soya (Japanese Ship) & & 206 & & & & & & & & & & & & & & & & & & & & & & \\
\hline Svalbard, Norway & & & & & & & 166 & 165 & 208 & 208 & 214 & 214 & 192 & 192 & 208 & 190 & 191 & 190 & 190 & 196 & 213 & 208 & 202 & 215 \\
\hline Sverdlovsk, USSR & 155 & & 160 & 161 & 168 & 164 & 170 & 177 & & & & & & & & & & & & & & & & \\
\hline Tahiti, Society Is. & & & & & & & & & & & & 219 & 195 & 193 & 195 & 197 & 197 & 211 & 199 & 200 & 201 & 202 & 203 & 203 \\
\hline Talara, Peru & 158 & 155 & 158 & 158 & 158 & 159 & 163 & 163 & 163 & 165 & 165 & 165 & 166 & 168 & 163 & 170 & 170 & 170 & 171 & 175 & 176 & 177 & 178 & 176 \\
\hline Tananrasset, French $W$. Africa & 216 & 216 & 216 & 216 & & & 219 & 219 & 213 & & 219 & & & 193 & 195 & 197 & 197 & 211 & 199 & 201 & 201 & 202 & 203 & \\
\hline Tananarive, Madagascar & 216 & 216 & 216 & 216 & 216 & 216 & 219 & 219 & 213 & 213 & 219 & 219 & 195 & 194 & 195 & 197 & 197 & 211 & 200 & 200 & 201 & 202 & 203 & 203 \\
\hline Terre Adelie & 216 & & 196 & 199 & 198 & 193 & 211 & 211 & 211 & 211 & 211 & 211 & 205 & 212 & 212 & 212 & 212 & 212 & 217 & 217 & 217 & 217 & 217 & 217 \\
\hline Thule, Greenland & 152 & 153 & 155 & 155 & 157 & 158 & 160 & 161 & 162 & 162 & 164 & 165 & 165 & 167 & 163 & 168 & 170 & 173 & 175 & 175 & 175 & 174 & 174 & 177 \\
\hline Tiruchy, India & & & & & & 169 & 176 & 177 & 213 & 214 & 214 & $2 \overline{14}$ & & 191 & & & & & & & 215 & & & \\
\hline Tokyo, Japan & 156 & 155 & 157 & 157 & 158 & 161 & 163 & 162 & 163 & 164 & 165 & 166 & 168 & 169 & 170 & 178 & 172 & 173 & 179 & 179 & 180 & 180 & 182 & 183 \\
\hline Tomsk, USSR & 155 & & 157 & 161 & & 164 & 170 & & & & & & & & & & & & & & & & & \\
\hline Tortosa, Spain & & & & & & & 174 & 164 & & & & 166 & & & & & 174 & & & 179 & 179 & & & \\
\hline Townsville, Australia & 158 & 158 & 158 & 158 & 158 & 160 & 160 & 162 & 164 & 164 & 166 & 168 & 169 & 183 & 176 & 177 & 178 & 173 & 175 & 190 & 215 & 180 & 181 & 182 \\
\hline Trelew, Argentina & & & & & & & & & & & & & & & & 194 & 216 & 219 & 192 & 191 & 191 & 215 & 215 & 215 \\
\hline Trivandrum, India & & & & & & 171 & 176 & 177 & 213 & 214 & 214 & 214 & & 191 & & & & & & & 215 & & & \\
\hline Tronso, Norway & 154 & 155 & 156 & 157 & 158 & 159 & 159 & 160 & 161 & 163 & 163 & 165 & 166 & 168 & 176 & 177 & 179 & 178 & 173 & 179 & 176 & 180 & 182 & 182 \\
\hline Tsumeb, South W. Africa & & & & & & & 171 & 212 & 215 & 212 & 212 & 212 & 198 & 195 & $19{ }^{\prime}$ & 197 & 193 & 198 & 198 & 200 & 200 & 202 & 203 & 203 \\
\hline Tucunan, Argentina & & & & & & & 170 & 177 & 214 & 214 & 214 & 214 & 216 & 189 & 216 & 190 & 215 & 215 & 192 & 192 & 192 & 215 & 215 & 215 \\
\hline Uppsala, Sweden & 154 & 152 & 153 & 156 & 155 & 159 & 158 & 158 & 160 & 161 & 164 & 165 & 168 & 165 & 169 & 170 & 168 & 178 & 179 & 179 & 180 & 180 & 182 & 183 \\
\hline Ushuaia, Argentina & & & & & & & & & & & 215 & & & 193 & 191 & & & & 192 & & 192 & 215 & 215 & 215 \\
\hline Victoria, Canada & & & & & & & 174 & 163 & 163 & 164 & 169 & 167 & & 215 & 215 & 215 & 215 & & 216 & & & 216 & 216 & 191 \\
\hline Wakkanai, Japan & 155 & 155 & 157 & 157 & 158 & 161 & 163 & 162 & 163 & 164 & 165 & 166 & 168 & 168 & 170 & 178 & 172 & 173 & 179 & 179 & 180 & 180 & 182 & 183 \\
\hline Washington, D. C. & 150 & 151 & 152 & 153 & 154 & 155 & 158 & 160 & 161 & 162 & 163 & 163 & 166 & 166 & 166 & 167 & 169 & 169 & 170 & 171 & 172 & 173 & 174 & 175 \\
\hline Watheroo, W. Australia & 154 & 155 & 156 & 156 & 157 & 159 & 161 & 160 & 163 & 164 & 166 & 166 & 168 & 169 & 176 & 187 & 172 & 178 & 178 & 175 & 176 & 181 & 183 & 182 \\
\hline White Sands, New Mexico & 152 & 153 & 154 & 156 & 156 & 158 & 159 & 160 & 162 & 163 & 164 & 165 & 167 & 167 & 168 & 168 & 170 & 170 & 172 & 172 & 173 & 174 & 176 & 177 \\
\hline Wilkes Station, Antarctica & & & & & & & 177 & 177 & 178 & 178 & 178 & 178 & 180 & 182 & & & 183 & 183 & 184 & 184 & 184 & & 184 & 184 \\
\hline $\begin{array}{l}\text { Winnipeg, Canada } \\
\text { Yakutsk, USSR }\end{array}$ & 154 & $\begin{array}{l}155 \\
157\end{array}$ & 156 & $\begin{array}{l}157 \\
167\end{array}$ & $\begin{array}{l}158 \\
163\end{array}$ & 159 & 159 & 162 & 163 & 163 & 165 & 165 & 166 & 166 & 177 & 177 & 172 & 178 & 173 & 179 & 179 & 181 & 182 & 182 \\
\hline $\begin{array}{l}\text { Yakut sk, USSR } \\
\text { Yamagawa, Japan }\end{array}$ & 156 & $\begin{array}{l}157 \\
155\end{array}$ & $\begin{array}{l}157 \\
157\end{array}$ & $\begin{array}{l}167 \\
157\end{array}$ & $\begin{array}{l}163 \\
158\end{array}$ & $\begin{array}{l}164 \\
161\end{array}$ & 163 & 162 & 163 & 164 & 165 & 166 & 168 & 163 & & 178 & 172 & 173 & & & & & & \\
\hline $\begin{array}{l}\text { Yamagawa, Japan } \\
\text { Yellowknife, Canada }\end{array}$ & & נת & 137 & 1 & & 101 & & & & & 215 & 215 & & & 194 & 170 & 172 & 173 & $\begin{array}{l}179 \\
215\end{array}$ & $\begin{array}{l}179 \\
215\end{array}$ & $\begin{array}{l}180 \\
215\end{array}$ & $\begin{array}{l}180 \\
215\end{array}$ & $\begin{array}{l}182 \\
192\end{array}$ & $\begin{array}{l}183 \\
193\end{array}$ \\
\hline Yuzhno-Sakhalinsk, USSR & & & 157 & 167 & 168 & & & & & & & & & & & & & & & & & & & \\
\hline
\end{tabular}

\begin{tabular}{|c|c|c|c|c|c|c|c|c|c|c|c|c|c|c|c|c|c|c|c|c|c|c|c|c|}
\hline \multirow[t]{2}{*}{ Station } & \multicolumn{12}{|c|}{1959} & \multicolumn{12}{|c|}{1960} \\
\hline & J & F & M & A & $\mathrm{M}$ & J & $\mathrm{Jy}$ & A & S & 0 & $\mathrm{~N}$ & D & $\mathrm{J}$ & F & $M$ & A & M & $\mathrm{J}$ & Jy & A & S & 0 & $\mathrm{~N}$ & D \\
\hline Adak, Alaska & 179 & 179 & 181 & 182 & 183 & 183 & 185 & 186 & 187 & 187 & 187 & 188 & 189 & 190 & 192 & 195 & 196 & 215 & 210 & 211 & 212 & 212 & 202 & 203 \\
\hline Ahmedabad, India & 205 & 206 & 210 & 213 & 218 & 220 & 218 & 217 & 220 & 220 & 220 & 220 & & & & 219 & & & & & & & & \\
\hline Akita, Japan & 185 & 186 & 186 & 187 & 188 & 188 & 207 & 210 & 215 & 209 & 205 & 204 & 206 & 193 & 194 & 195 & 196 & 198 & 199 & 199 & 200 & 201 & 202 & 203 \\
\hline Anchorage, Alaska & 179 & 179 & 181 & 181 & 183 & 185 & 186 & 186 & 184 & 187 & 187 & 188 & 189 & 189 & 190 & 195 & 215 & 216 & 210 & 211 & 212 & 212 & 203 & 203 \\
\hline Baguio, P. I. & 179 & 179 & 181 & 182 & 183 & 186 & 183 & 186 & 186 & 187 & 187 & 188 & 189 & 189 & 190 & 207 & 215 & 216 & 210 & 211 & 212 & 212 & 213 & 214 \\
\hline Baker Lake, Canada & 184 & 185 & & & & & & & & & & & & & & & & & & & & & & \\
\hline Bogota, Colombia & 184 & 184 & 185 & 184 & 184 & & 189 & 190 & 192 & 189 & 188 & 190 & & & & & & & & 217 & & & & \\
\hline Bonbay, India & 205 & 206 & 210 & 213 & 218 & 220 & 218 & 217 & 220 & 220 & 220 & 220 & & & & & & & & & & & & \\
\hline Boulder, Colorado & 189 & 189 & 189 & 189 & 189 & 189 & 189 & 187 & & 188 & 188 & 189 & 189 & 189 & 192 & 194 & 193 & 215 & 210 & 211 & 212 & 213 & 202 & 203 \\
\hline Brisbane, Australia & 184 & 184 & 185 & 185 & 188 & 189 & 204 & 190 & 192 & 204 & 204 & 191 & 204 & 205 & 196 & & & 197 & & 199 & 200 & 201 & & \\
\hline Budapest, Hungary & & 193 & 189 & 185 & 188 & 198 & & 190 & 193 & 201 & 204 & 203 & & & & & & & & & & & & \\
\hline Buenos Aires, Argentina & 192 & & 194 & 195 & 197 & 198 & 198 & 199 & 200 & 201 & 202 & 204 & & & & & 208 & & & & & & & \\
\hline Bunia, Congo & 186 & 186 & 185 & 187 & 188 & 189 & & 191 & 193 & 209 & 204 & 202 & 192 & 193 & 195 & 196 & 196 & & & & & & & \\
\hline Byrd Station & 190 & 191 & 193 & 193 & 194 & 197 & 198 & 199 & 200 & 192 & 203 & 209 & 212 & 212 & 206 & 207 & 216 & 216 & 210 & 211 & 213 & 213 & 213 & 214 \\
\hline Calcutta, Iniia & 205 & 206 & 207 & & & & & 217 & 220 & 220 & 220 & 220 & & & & & & & & & & & & \\
\hline Camphell I. & 204 & 206 & 207 & 218 & 218 & 218 & 218 & 218 & 218 & 218 & 218 & 218 & & & & & & & & & & & & \\
\hline Canberra, Australia & 192 & 193 & 194 & 195 & 196 & 198 & 192 & 199 & 200 & 201 & 202 & 203 & & & & & & & & & & & & \\
\hline Cape Hallett & 185 & 220 & 220 & 220 & 220 & 220 & 220 & 220 & 220 & 220 & 220 & 220 & & & & & & & & & & & & \\
\hline $\begin{array}{l}\text { Capetown, Union of S. Africa } \\
\text { Chimbote, Peru }\end{array}$ & $\begin{array}{l}184 \\
180\end{array}$ & $\begin{array}{l}184 \\
181\end{array}$ & 186 & $\begin{array}{l}188 \\
18 ?\end{array}$ & $\begin{array}{l}196 \\
183\end{array}$ & 198 & 199 & 199 & 200 & 201 & 202 & 203 & & & & & & & & & & & & \\
\hline Chrdstchurch, New Zealand & 185 & 206 & 207 & 219 & 219 & 219 & 219 & 219 & 219 & 218 & 218 & 218 & & & & & & & & & & & & \\
\hline Churchill, Canada & 184 & 185 & 187 & 187 & 209 & 211 & 205 & 210 & & 201 & 201 & 205 & 192 & 207 & 195 & 195 & 196 & 197 & 198 & 199 & 200 & 201 & 202 & 203 \\
\hline
\end{tabular}




\begin{tabular}{|c|c|c|c|c|c|c|c|c|c|c|c|c|c|c|c|c|c|c|c|c|c|c|c|c|}
\hline \multirow[t]{2}{*}{ 7wh } & \multicolumn{12}{|c|}{ 1. . 9} & \multicolumn{12}{|c|}{1960} \\
\hline & 1 & 5 & 11 & $\mathrm{~A}$ & M & i & Jy & A & s & 0 & N & D & $\mathrm{J}$ & F & $\mathrm{M}$ & $\mathrm{A}$ & $\mathrm{M}$ & $\mathrm{J}$ & Jy & A & s & 0 & $\mathrm{~N}$ & D \\
\hline $\begin{array}{l}\text { Watheroo, W. Australia } \\
\text { Whice sands, New Mexico } \\
\text { Wilkes Station. Antarctica } \\
\text { Winnipeg, Canada } \\
\text { Yamacawa, Japan } \\
\text { Yellowknife, Canada }\end{array}$ & $\begin{array}{l}185 \\
179 \\
184 \\
188 \\
155 \\
192\end{array}$ & $\begin{array}{l}184 \\
179 \\
219 \\
185\end{array}$ & $\begin{array}{l}181 \\
212 \\
185 \\
186\end{array}$ & $\begin{array}{l}182 \\
214 \\
187\end{array}$ & $\begin{array}{l}183 \\
214 \\
219 \\
188\end{array}$ & $\begin{array}{r}185 \\
212 \\
188\end{array}$ & $\begin{array}{l}187 \\
212 \\
209 \\
207\end{array}$ & $\begin{array}{l}186 \\
199 \\
199 \\
210\end{array}$ & $\begin{array}{l}187 \\
200 \\
200 \\
215\end{array}$ & $\begin{array}{l}187 \\
219 \\
201 \\
209\end{array}$ & $\begin{array}{l}188 \\
212 \\
209 \\
205\end{array}$ & $\begin{array}{l}189 \\
208 \\
204\end{array}$ & $\begin{array}{l}189 \\
192 \\
206\end{array}$ & $\begin{array}{l}191 \\
205 \\
193 \\
193\end{array}$ & $\begin{array}{l}193 \\
195 \\
194\end{array}$ & $\begin{array}{l}193 \\
195 \\
195\end{array}$ & $\begin{array}{l}208 \\
196 \\
196\end{array}$ & $\begin{array}{l}216 \\
197 \\
198\end{array}$ & $\begin{array}{l}210 \\
198 \\
199\end{array}$ & $\begin{array}{l}211 \\
199 \\
199\end{array}$ & $\begin{array}{l}201 \\
200 \\
200\end{array}$ & $\begin{array}{l}201 \\
201 \\
201\end{array}$ & $\begin{array}{l}202 \\
202 \\
202\end{array}$ & $\begin{array}{l}203 \\
203 \\
203\end{array}$ \\
\hline
\end{tabular}

\begin{tabular}{|c|c|c|c|c|c|c|c|c|c|c|c|c|c|c|c|c|c|c|c|c|c|c|c|c|}
\hline \multirow[t]{2}{*}{ Statiun } & \multicolumn{12}{|c|}{1901} & \multicolumn{12}{|c|}{1962} \\
\hline & 3 & $F$ & M & A & $M$ & J & Jv & A & s & 0 & $\mathrm{~N}$ & D & J & F & M & A & M & $\mathrm{J}$ & Jy & A & s & 0 & $\mathrm{~N}$ & D \\
\hline $\begin{array}{l}\text { Idai, Mash } \\
\text { Akita, Japan } \\
\text { Anchorage, Alaska } \\
\text { Baguio, P. I. } \\
\text { Boulder, Colorado } \\
\text { Brisbane, Australid } \\
\text { Byrd Station } \\
\text { Canberra, Australiu } \\
\text { Capetown, Union of S. Africa } \\
\text { Christchurch, New Zealand }\end{array}$ & $\begin{array}{l}214 \\
204 \\
214 \\
214 \\
-14 \\
204 \\
205 \\
\end{array}$ & $\begin{array}{l}214 \\
205 \\
214 \\
205 \\
214 \\
214 \\
205 \\
206 \\
\end{array}$ & $\begin{array}{l}214 \\
206 \\
216 \\
206 \\
216 \\
214 \\
206 \\
206 \\
\end{array}$ & $\begin{array}{l}216 \\
209 \\
216 \\
217 \\
\\
207 \\
\end{array}$ & $\begin{array}{l}216 \\
208 \\
215 \\
208 \\
216 \\
205 \\
\\
208 \\
208 \\
\end{array}$ & $\begin{array}{l}213 \\
210 \\
216 \\
209 \\
215 \\
20 y \\
\\
209 \\
209 \\
\end{array}$ & $\begin{array}{l}210 \\
211 \\
210 \\
210 \\
210 \\
210 \\
210 \\
\end{array}$ & $\begin{array}{l}212 \\
211 \\
212 \\
211 \\
211 \\
211 \\
211\end{array}$ & 213 & 217 & 218 & 218 & $\begin{array}{l}220 \\
220\end{array}$ & & & & & & & & & & & \\
\hline $\begin{array}{l}\text { Churchill, Canada } \\
\text { Concepcion, Chile } \\
\text { De Bilt, Holland } \\
\text { Dourbes, Bel Mium } \\
\text { E1 Cerillo, Mexico } \\
\text { Fairbanks, Alaska } \\
\text { Falkland Is. } \\
\text { Fomosa, China } \\
\text { Ft. Monmouth, Wew Jersey } \\
\text { Godhavn, Greenland } \\
\end{array}$ & $\begin{array}{l}204 \\
214 \\
204 \\
206 \\
214 \\
204 \\
218 \\
217 \\
\end{array}$ & $\begin{array}{l}205 \\
214 \\
206 \\
200 \\
205 \\
214 \\
20 \\
218 \\
214 \\
\end{array}$ & $\begin{array}{l}206 \\
217 \\
206 \\
207 \\
206 \\
214 \\
207 \\
206 \\
218 \\
216 \\
\end{array}$ & $\begin{array}{l}207 \\
217 \\
207 \\
\\
216 \\
207 \\
207 \\
219 \\
217 \\
\end{array}$ & $\begin{array}{l}208 \\
208 \\
208 \\
216 \\
208 \\
219 \\
217 \\
\end{array}$ & $\begin{array}{l}209 \\
209 \\
209 \\
209 \\
216 \\
210 \\
209 \\
219 \\
217 \\
\end{array}$ & $\begin{array}{l}210 \\
210 \\
210 \\
216 \\
210 \\
210 \\
217\end{array}$ & $\begin{array}{l}211 \\
211 \\
220 \\
211 \\
\end{array}$ & 212 & 213 & $\begin{array}{r}220 \\
\end{array}$ & 220 & & & & & & & & & & & & \\
\hline $\begin{array}{l}\text { Grand Bahama I. } \\
\text { Graz, Austria } \\
\text { Hobart, Tasmania } \\
\text { Huancayo, Peru } \\
\text { Invemess. Scotland } \\
\text { Johannesburg, Union of B. Aftua } \\
\text { Kiruna, Sweden } \\
\text { La Paz, Bolivia } \\
\text { Lulea, Sweden } \\
\text { Lwiro, Congo }\end{array}$ & $\begin{array}{l}204 \\
204 \\
204 \\
204 \\
204\end{array}$ & $\begin{array}{l}205 \\
204 \\
205 \\
205 \\
205 \\
217 \\
205\end{array}$ & $\begin{array}{l}218 \\
206 \\
206 \\
206 \\
206 \\
206 \\
217 \\
206\end{array}$ & $\begin{array}{l}218 \\
207 \\
206 \\
207 \\
207 \\
207 \\
207\end{array}$ & $\begin{array}{l}219 \\
208 \\
208 \\
207 \\
208 \\
208 \\
208\end{array}$ & $\begin{array}{l}209 \\
209 \\
208 \\
210 \\
209 \\
209 \\
209\end{array}$ & $\begin{array}{l}210 \\
210 \\
208 \\
210 \\
210 \\
210\end{array}$ & $\begin{array}{l}211 \\
211 \\
217 \\
211 \\
211 \\
211 \\
\end{array}$ & 213 & 218 & $21 \overline{8}$ & & 220 & & & & & & & & & & & \\
\hline $\begin{array}{l}\text { Lycksele, Sweden } \\
\text { Maui, Hawaii } \\
\text { Hundaring, W. Australıa } \\
\text { Narssarssuaq, Greenland } \\
\text { Numijarvi, Finland } \\
\text { Ottawa, Canada } \\
\text { Point Barrow, Alaska } \\
\text { Pole Station } \\
\text { Pruhonice, Czechoslovakia } \\
\text { Resolute Bay, Canada }\end{array}$ & $\begin{array}{l}204 \\
217 \\
204 \\
218 \\
204 \\
204 \\
219 \\
219 \\
204 \\
\end{array}$ & $\begin{array}{l}205 \\
217 \\
205 \\
218 \\
205 \\
205 \\
219 \\
\\
205 \\
\end{array}$ & $\begin{array}{l}206 \\
217 \\
218 \\
206 \\
206 \\
219 \\
206 \\
\end{array}$ & $\begin{array}{l}207 \\
217 \\
207 \\
219 \\
207 \\
207 \\
219 \\
207 \\
209 \\
\end{array}$ & $\begin{array}{l}208 \\
220 \\
208 \\
218 \\
209 \\
208 \\
218 \\
208 \\
208 \\
\end{array}$ & $\begin{array}{l}209 \\
220 \\
209 \\
219 \\
209 \\
209 \\
218 \\
209 \\
209 \\
\end{array}$ & $\begin{array}{l}210 \\
220 \\
210 \\
219 \\
210 \\
210 \\
219 \\
210 \\
210 \\
\end{array}$ & $\begin{array}{l}211 \\
220 \\
211 \\
219 \\
211 \\
211 \\
218 \\
211 \\
211 \\
\end{array}$ & 220 & 219 & 219 & 220 & 220 & 220 & 220 & & & & & & & & & \\
\hline $\begin{array}{l}\text { Reykjavik, Iceland } \\
\text { Rome, Italy } \\
\text { St. John's, Newfoundland } \\
\text { Schwarzenburg, Switzerland } \\
\text { Singapore, Britisi Malaya } \\
\text { Slough, England } \\
\text { Sodankyla, Finland } \\
\text { Sottens, Switzerland } \\
\text { Talara, Peru } \\
\text { Thule, Greenland. }\end{array}$ & $\begin{array}{l}220 \\
204 \\
204 \\
204 \\
\\
204 \\
204 \\
2017 \\
219 \\
\end{array}$ & $\begin{array}{l}220 \\
205 \\
205 \\
205 \\
205 \\
205 \\
205 \\
205 \\
206 \\
219 \\
\end{array}$ & $\begin{array}{l}220 \\
206 \\
206 \\
206 \\
206 \\
207 \\
206 \\
206 \\
204 \\
219 \\
\end{array}$ & $\begin{array}{l}220 \\
207 \\
207 \\
207 \\
207 \\
207 \\
207 \\
200 \\
219 \\
\end{array}$ & $\begin{array}{l}220 \\
208 \\
208 \\
208 \\
\\
208 \\
208 \\
207 \\
219 \\
\end{array}$ & $\begin{array}{l}220 \\
209 \\
209 \\
209 \\
210 \\
209 \\
209 \\
209 \\
208 \\
219\end{array}$ & \begin{tabular}{|l|}
220 \\
211 \\
210 \\
211 \\
210 \\
209 \\
210 \\
211 \\
217 \\
219 \\
\end{tabular} & $\begin{array}{l} \\
211 \\
211 \\
211 \\
211 \\
211 \\
\\
211 \\
217 \\
219 \\
\end{array}$ & . & $\begin{array}{l}217 \\
219\end{array}$ & $\begin{array}{l}218 \\
220 \\
\end{array}$ & $\begin{array}{l}218 \\
219 \\
\end{array}$ & & & & & & & & & & & & \\
\hline $\begin{array}{l}\text { Tokyo, Japan } \\
\text { Townsville, Australia } \\
\text { Tromso, Norway } \\
\text { Uppsala, Sweden } \\
\text { Wakkanai, Japan } \\
\text { Washington, D. C. } \\
\text { White Sands, New Mexico } \\
\text { Winnıpeg, Canada } \\
\text { Yamagawa, Japan }\end{array}$ & $\begin{array}{l}204 \\
204 \\
204 \\
204 \\
203 \\
204 \\
204\end{array}$ & $\begin{array}{l}205 \\
205 \\
205 \\
205 \\
205 \\
205 \\
205 \\
205\end{array}$ & $\begin{array}{l}206 \\
206 \\
206 \\
206 \\
206 \\
205 \\
206 \\
106\end{array}$ & $\begin{array}{l}209 \\
207 \\
207 \\
209 \\
205 \\
220 \\
207 \\
209\end{array}$ & $\begin{array}{l}208 \\
208 \\
208 \\
208 \\
208 \\
207 \\
208 \\
208\end{array}$ & $\begin{array}{l}210 \\
709 \\
309 \\
209 \\
210 \\
208 \\
209 \\
210\end{array}$ & $\begin{array}{l}211 \\
211 \\
210 \\
211 \\
208 \\
210 \\
211\end{array}$ & $\begin{array}{l}211 \\
211 \\
211 \\
211 \\
209 \\
211 \\
211\end{array}$ & 212 & 218 & 217 & 218 & 218 & 220 & & & & & & & & & & \\
\hline
\end{tabular}

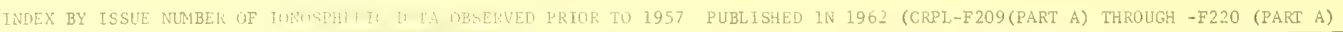

\begin{tabular}{|c|c|c|c|c|c|c|c|c|c|c|c|c|c|c|c|c|c|c|c|c|c|c|c|}
\hline \multirow[t]{2}{*}{ Station } & \multicolumn{11}{|c|}{1956} & \multicolumn{12}{|c|}{1955} \\
\hline & J & F & M & A & M & J & Jy A & s & 0 & N & D & J & $F$ & M & A. & M & J & Jy & A & s & 0 & $\mathrm{~N}$ & D \\
\hline $\begin{array}{l}\text { Campbell I. } \\
\text { Canbetra, Australia } \\
\text { Casablanca, Morocco } \\
\text { Dakar, French W. Africa } \\
\text { Djibout i, French Somaliland }\end{array}$ & & & & & & & & $\begin{array}{rr}215 & \\
215 & 2 \\
215 & 2 \\
215 & 2\end{array}$ & $\begin{array}{l}215 \\
215 \\
215\end{array}$ & $\begin{array}{l}215 \\
213 \\
215\end{array}$ & $\begin{array}{l}213 \\
215 \\
215\end{array}$ & & & & & & & & & 213 & 213 & & \\
\hline $\begin{array}{l}\text { Freiburs, Germany } \\
\text { Kerguelen I. }\end{array}$ & 213 & $21 \overline{3}$ & 2132 & & 2132 & 213 & & 2152 & 2152 & 215 & 215 & & & & & & & & & 213 & 213 & 213 & 213 \\
\hline $\begin{array}{l}\text { Lwiro, Congo } \\
\text { Poitiers, France } \\
\text { Sito Paulo, Brazil }\end{array}$ & 219 & 219 & 2192 & 2192 & 2193 & 219 & & & 2152 & 2152 & $\begin{array}{l}215 \\
215 \\
\end{array}$ & & & 2112 & 2112 & 2112 & 211 & 213 & 213 & 213 & & & \\
\hline $\begin{array}{l}\text { Tananrasset, French } 4 . \text { Africd } \\
\text { Tananarive, Mudagascal } \\
\text { Terra Adelie }\end{array}$ & & & & & & & & $\begin{array}{rr}2 & 2 \\
215 & 2 \\
215 & 2\end{array}$ & $\begin{array}{ll}215 & 2 \\
215 & 2 \\
215 & 2\end{array}$ & $\begin{array}{l}2152 \\
215 \\
215\end{array}$ & $\begin{array}{l}215 \\
215 \\
215\end{array}$ & & & & & & & & & & & & \\
\hline & \multicolumn{11}{|c|}{1954} & \multicolumn{12}{|c|}{1953} \\
\hline $\begin{array}{l}\text { Freibur:, fermany } \\
\text { Lulea, weden } \\
\text { Lwiro, con to }\end{array}$ & $1 '$ & & & & & $\begin{array}{l}210 \\
215\end{array}$ & $\begin{array}{l}210 \\
\because 15,211 \\
215\end{array}$ & $\begin{array}{l}2122 \\
2152\end{array}$ & $\begin{array}{l}2123 \\
2152\end{array}$ & $\begin{aligned} 2123 \\
2152\end{aligned}$ & $\begin{array}{l}212 \\
215\end{array}$ & & & & & & 211 & & 214 & 214 & 214 & 214 & 214 \\
\hline
\end{tabular}




\section{CRPL Reports}

[A detailed list of CRPL publications is available from the Central Radio Propagation Laboratory upon request] Daily:

Radio disturbance forecasts, every half hour from broadcast stations WWV and WWVH of the National Bureau of Standards.

Telephoned and telegraphed reports of ionospheric, solar, geomagnetic, and radio propagation data.

Weekly:

CRPL-J. North Atlantic Radio Propagation Forecast.

CRPL-Jp. North Pacific Radio Propagation Forecast.

Semimonthly:

CRPL-Ja. Semimonthly Frequency Revision Factors For CRPL Basic Radio Propagation Prediction Reports.

Monthly:

CRPL-D. Basic Radio Propagation Predictions-Three months in advance. (Dept. of the Army, TB 11499-, monthly supplements to TM 11-499; Dept. of the Air Force, TO 31-3-28 series). On sale by Superintendent of Documents. Members of the Armed Forces should address cognizant military office.

CRPL-F. (Part A). Ionospheric Data.

(Part B). Solar-Geophysical Data.

Limited distribution. These publications are in general disseminated only to those individuals or scientific organizations which collaborate in the exchange of ionospheric, solar, geomagnetic, or other radio propagation data.

Catalog of Data:

A catalog of records and data on file at the U. S. IGY. World Data Center A for Airglow and Ionosphere, Boulder Laboratories, National Bureau of Standards, which includes a fee schedule to cover the cost of supplying copies, is available upon request.

The publications listed above may be obtained without charge from the Central Radio Propagation Laboratory, National Bureau of Standards, Boulder Laboratories, Boulder, Colorado, unless otherwise indicated. Please note that the $\mathrm{F}$ series is not generally available.

Circulars of the National Bureau of Standards pertaining to Radio Sky Wave Transmission:

NBS Circular 462.

NBS Circular 465.

Ionospheric Radio Propagation. \$1.25.

NBS Circular 557 .

NBS Circular 582.

Instructions for the Use of Basic Radio Propagation Predictions. 30 cents.

Worldwide Radio Noise Levels Expected in the Frequency Band 10 Kilocycles to 100 megacycles. 30 cents.

Worldwide Occurrence of Sporadic $E$. $\$ 3.25$.

These Circulars are on sale by the Superintendent of Documents, U. S. Government Printing Office, Washington 25, D. C. Members of the Armed Forces should address the respective military office having cognizance of radio wave propagation.

Selected Technical Notes of the National Bureau of Standards:

NBS Tech. Note 2. PB151361. World Maps of F2 Critical Frequencies and Maximum Usable Frequency Factors. $\$ 3.50 . \quad$ PB151361-2. \$3.50.

NBS Tech. Note 13. PB151372. Technical Considerations Leading to an Optimum Allocation of Radio Frequencies in the Band 25 to 60 Mc. $\$ 2.50$.

NBS Tech. Note 18. PB151377. Radio Noise Data for the IGY. \$2.50.

18-2. PB151377-2. Quarterly Radio Noise Data (Mar.-May 1959). \$1.00.

18-3. PB151377-3.

18-4. PB151377-4, etc. (Sept.-Nov. 1959). \$1.50.

NBS Tech. Note $31 . \quad$ PB151390. An Atlas of Oblique-Incidence Ionograms. \$2.25.

NBS Tech. Note 40-1. PB151399-1. Mean Electron Density Variations of the Quiet Ionosphere, 1: March 1959. $\$ 1.25$.

40-2. PB151399-2, etc. 2: April 1959. \$1.25.

NBS Tech. Note 117. PB161618. Variations in Frequency of Occurrence of Sporadic E, 1949-1959. $\$ 0.75$. These Technical Notes are on sale by the Office of Technical Services, U. S. Department of Commerce, Washington 25, D. C. Order by PB number. 
